\title{
An evaluation of preliminary outcomes and characteristics of peer support within a community based weight management program
}

John Spencer Ingels

Follow this and additional works at: https://researchrepository.wvu.edu/etd

\section{Recommended Citation}

Ingels, John Spencer, "An evaluation of preliminary outcomes and characteristics of peer support within a community based weight management program" (2018). Graduate Theses, Dissertations, and Problem Reports. 7289.

https://researchrepository.wvu.edu/etd/7289

This Dissertation is protected by copyright and/or related rights. It has been brought to you by the The Research Repository @ WVU with permission from the rights-holder(s). You are free to use this Dissertation in any way that is permitted by the copyright and related rights legislation that applies to your use. For other uses you must obtain permission from the rights-holder(s) directly, unless additional rights are indicated by a Creative Commons license in the record and/ or on the work itself. This Dissertation has been accepted for inclusion in WVU Graduate Theses, Dissertations, and Problem Reports collection by an authorized administrator of The Research Repository @ WVU.

For more information, please contact researchrepository@mail.wvu.edu. 
An evaluation of preliminary outcomes and characteristics of peer support within a community based weight management program

John Spencer Ingels, M.A.

Dissertation submitted to the College of Physical Activities and Sport Sciences at West Virginia University

in partial fulfillment of the requirements for the degree of

Doctor of Philosophy in Sport and Exercise Psychology

Sam Zizzi, Ed.D., Chair

Dana K. Voelker, Ph.D.

Abhik Roy, Ph.D.

Karen Rambo-Hernandez, Ph.D.

Department of Sport Sciences

Morgantown, WV

2018

Keywords: peer support, weight management, social support, hierarchical linear modeling, propensity score matching

Copyright 2018 Ingels 


\begin{abstract}
An evaluation of preliminary outcomes and characteristics of peer support within a community based weight management program

John Spencer Ingels, M.A.

Peer support research in weight management has been limited and peer training has varied. The purpose of this study was to examine the feasibility and preliminary outcomes of pairing peers, without training, in a community-based weight management program and to explore characteristics influencing the peer relationship. Peers maintained regular contact, were satisfied, and reported increased support. Hierarchical Linear Modeling was run to compare weight loss outcomes. A comparison of weight loss outcomes with a propensity score matched group who did not work with a peer, did not find any significant differences in weight loss. Cohen's d was calculated to explore effect of peer request, BMI and age difference of peer pairings, revealing several moderate large effects, indicating that the peer relationship differed based on peer characteristics. Finally, peer pairings whose BMI differed by over 10 units lost significantly more weight than peers who weight differed by less than 10 units. Despite a lack of weight-loss at three months, a majority of peers were satisfied with peer support, contacted each other regularly, and reported increased support. The results suggest that intensive training may not be necessary for peers to provide impactful support in weight management.
\end{abstract}


Table of Contents

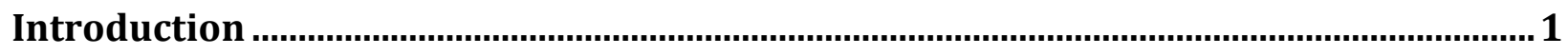

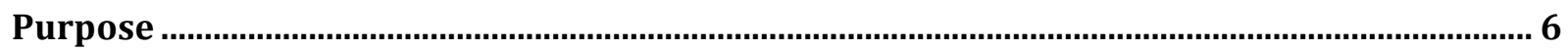

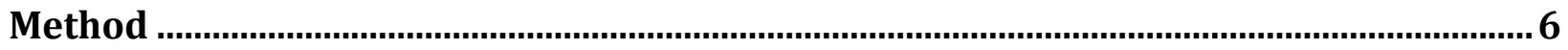

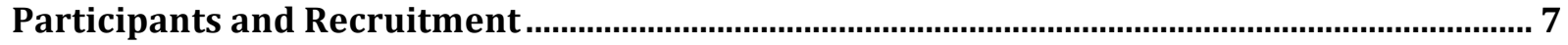

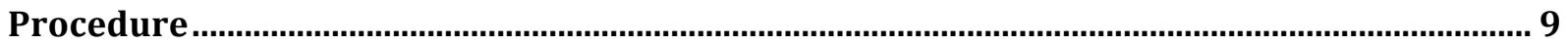

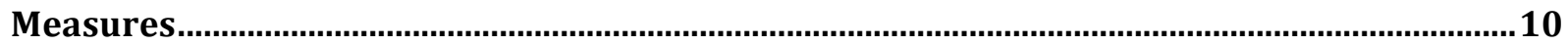

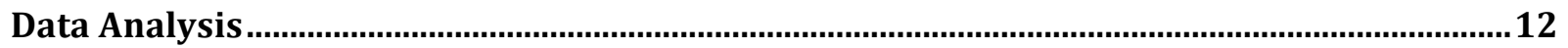

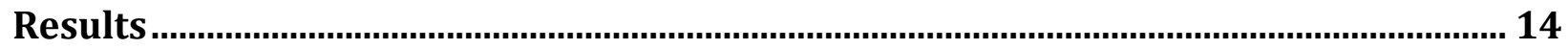

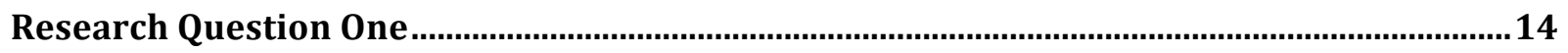

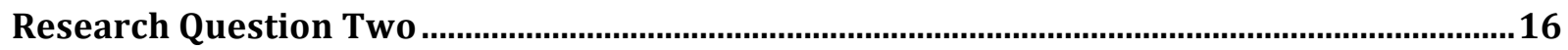

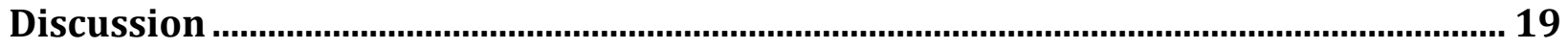

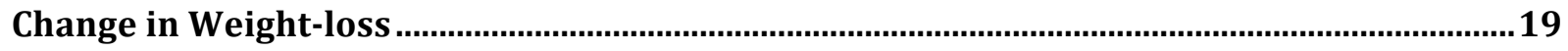

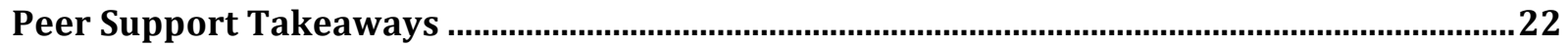

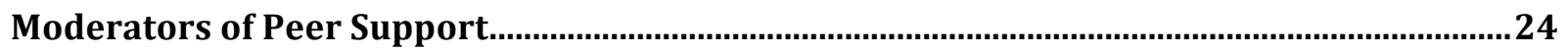

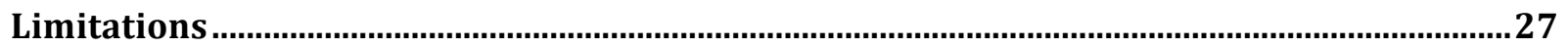

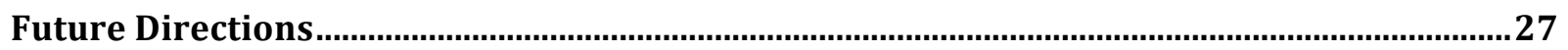

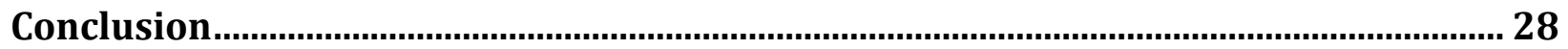

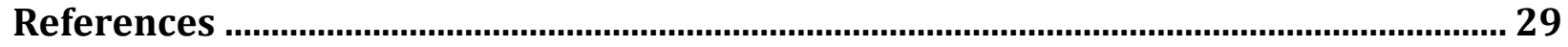

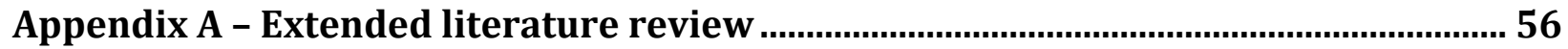

Appendix B - Peer support enrollment script ...........................................................119

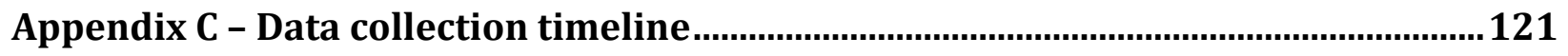

Appendix D - CES-Depression Scale .......................................................................... 122

Appendix E - Interpersonal Support Evaluation Scale .............................................124

Appendix F - Final HLM Model Structure and Interpretation ......................................126

Appendix G - Propensity Score Analysis Full Output ...................................................128

Appendix H - HLM Full Output.................................................................................. 134 


\section{Introduction}

A recent special edition of the American Psychologist highlighted the influence of close relationships, such as a spouse or friend, on health (Dunkel Schetter, 2017). One article from this issue reviewed the impact of close relationships on chronic disease management, specifically the influence these relationships have in making and sustaining health-behavior change (Martire \& Helgeson, 2017). An earlier large, prospective cohort study revealed that having a close friend who was obese increased personal likelihood of obesity by $171 \%$, which points to the influence that support networks can have on health, specifically weight gain (Christakis \& Fowler, 2007). Qualitative studies of participants in online weight-loss communities have shown that social support was perceived as an important part of successful weight-loss, as members helped each other stay motivated and shared relevant information, such as new healthy food options or strategies to increase daily activity (Hwang et al., 2010; Wang, Shih, \& Carroll, 2015). However, these participants also reported that support declined after they met their weight-loss goal.

Experimental designs have been applied to study the influence of support on weight-loss outcomes. One such study manipulated the level of support in a weight-loss intervention by having participants join with a friend or participate as part of a team (Wing \& Jeffery, 1999). The increased support resulted in fewer dropouts, greater weight-loss at four months, and a higher percentage of weight maintenance at ten months. Finally, two meta-analyses of weight-loss interventions found that interventions that included a social support component resulted in significantly greater weight-loss than the same intervention without support (Greaves et al., 2011; Huisman, Gucht, Dusseldorp, \& Maes, 2009). These results point to the benefit of social support within weight-loss and weight maintenance. The challenge is finding effective and efficient ways to provide support long-term. 
A growing body of research has explored the role peer support can play in improving health. Peers have been defined as people who share a similar background and who have experience with the same health condition or behavior change goal, such as obesity or diabetes (Dennis, 2003; Heisler, 2010). Peers have the benefit of mutual reciprocity; as one peer helps another with their condition they also help themselves stay healthy. Peers have been described as helping each other by providing emotional, informational, and appraisal support (Dennis, 2003; Heisler, 2010). Emotional support involves having someone to discuss personal difficulties and to provide support in the face of challenges that come with behavior change. Informational support is the sharing of relevant knowledge. Appraisal support focuses on helping individuals improve their self-evaluations by validating emotions, cognitions, and behaviors that are part of the process of behavior change. Further, the ability to empathize and connect with the life situation of one another improves their ability to provide support. Finally, the reciprocal nature of peer support creates motivation to stay in contact long-term, a situation that is unique to the peer support relationship. The finite resources of health care organizations limit their ability to provide long-term professional support (e.g. nurse, counselor, dietician) to patients working to make health behavior change.

A growing body of research focused on peer support intervention within type-two diabetes management has led to the completion of two meta-analyses. The first reviewed 13 randomized controlled trials (RCTs) and over 2,000 participants (Qi et al., 2015). They found an overall effect in favor of peer support (mean ES on A1C $=0.37$ ). This effect was greatest for both participants receiving individual intervention (mean $\mathrm{ES}=0.45$ ), and for participants who were in contact with their peers a minimum of once per month (mean ES $=0.40)$. Strongest results were seen in studies where peers contacted each other two or more times per month 
(mean ES $=0.43$ ). A second meta-analysis extended these findings in reviewing 20 RCTs with over 4,000 participants (Zhang, Yang, Sun, Fisher, \& Sun, 2016). Again a significant effect was found in favor of peer support (mean ES =0.14). Further, a three to six month intervention period was found to be most effective, especially when pairing peers who were starting the program together versus pairing with a peer who had already managed their blood sugar (mean $\mathrm{ES}=0.35)$. Finally, they found that peer support interventions provided in conjunction with a curriculum focused on diabetes management were the most successful. These results point to peer support as a promising and effective solution to extend support to patients with Type 2 diabetes. While some overlap exists between diabetes management and weight-loss, such as making changes to diet and physical activity, diabetes is unique as it requires increased focus on medical therapies (insulin injections) and poses greater risks for non-management (coma, death) that are not part of weight management.

To date there has been a lack of research studying peer support and weight management. Wilson and Pratt (1987) found that group delivered educational support from peers resulted in significantly greater weight-loss at eight weeks, but weight was regained over the final two months of the study when groups only met once per month. A more recent pilot study evaluated the feasibility of a group-based education program and telephone support from a trained peer health coach (Dutton, Phillips, Kukkamalla, Cherrington, \& Safford, 2015). Peer health coaches, who had lost weight in a prior program, received six hours of training on patient-centered communication, problem solving, and goal setting. Participants completing approximately $40 \%$ of the calls averaged a four percent loss in baseline weight and reported the calls helped in their weight-loss. Finally, a RCT looking at peer support delivered online through social media found 
that peer support was effective in lowering weight for those who actively participated in online chats (Kulik et al., 2015).

Two other RCTs have examined peer support within weight-loss. The first study (Leahey \& Wing, 2013) examined three types of support in promoting health-behavior change: professional ( $n=16$, support from health care providers), peer ( $n=16$, support from individuals in the same weight-loss intervention), and mentor ( $n=12$, support from someone who previously lost and maintained weight). Participants were obese with a Body Mass Index (BMI) between 30 and 40 and age between 40 and 60 years. All three groups lost a significant amount of weight, with participants in the peer and professional conditions losing significantly greater weight over six months (both over $-9 \%$ ) than those in the mentor condition $(-5.7 \%)$. Peer delivered support had a moderate effect $(d=0.67)$ on weight-loss compared to the mentor group. The second RCT compared effectiveness of peer support, professional support, and a standard group in maintaining weight-loss (Leahey et al., 2016). A group of 75 participants, who had recently lost at least five-percent of their weight in an eight-week weight-loss intervention, were randomized across the three conditions for the ten-month maintenance program. Results supported the ability of peers to provide similar support to professionals, as $76 \%$ of professional and $65 \%$ of peer supported participants maintained a five-percent loss, while only $37 \%$ of those in the standard condition maintained the same loss. Moreover, both the peer and professional groups continued to lose weight, while the standard group, on average, gained over three kilograms. Overall, these results represented a moderate effect size of 0.67 in favor of peer support versus standard care. While the number of studies of peer support within weight-loss is limited, the results indicate that peer support provides a moderate effect on participant weight-loss and is more cost effective than paying a healthcare professional to provide long-term support. 
To date, peer support research has primarily focused on controlled trials and further work is needed to study how peer support can be applied in community-based settings. In addition, the training provided to peer supporters has varied greatly across studies with training as short as one hour (e.g. Baumann, Frederick, Betty, Jospehine, \& Agatha, 2015; Leahey \& Wing, 2013) to spanning several days (e.g. Goldman et al., 2015; Thom et al., 2013; Yin et al., 2015). A review of peer support programs found a wide variety in training intensity, duration, and topics, and a lack of evaluation of training effectiveness (Tang, Ayala, Cherrington, \& Rana, 2011). It is unknown what training is needed, or if training is even necessary to improve health outcomes among peer supporters.

Finally, further research examining factors that influence the ability of peers to effectively support health-behavior change is needed. A large diabetes management study in China found that patients with higher negative emotions, like anxiety and depression, saw greater improvement from peer support (Chan et al., 2014). Two other studies, also within diabetes management, found that matching peers by age, gender, or ethnicity did not improve results, but found a significant effect for other characteristics such as disease related self-efficacy and selfmanagement skills (Moskowitz, Thom, Hessler, Ghorob, \& Bodenheimer, 2013; Rogers et al., 2014). Having a peer coach with higher diabetes distress and lower self-efficacy about diabetes management resulted in a greater improvement in blood sugar, which may be due to these peer coaches taking on a collaborative role instead of an expert role in their coaching. Third, coaches with lower depression scores were more effective. Finally, coach BMI was associated with improved outcomes. For coaches with a BMI under 35 a lower BMI was associated with improved outcomes, while for coaches with a BMI over 35 a higher BMI was associated with 
improved outcomes. These results warrant further research to understand how BMI influences the peer support relationship.

\section{Purpose}

The literature has shown positive effects on health outcomes for individuals participating in peer support programs, however most of the research has focused on diabetes management and the training delivered to peers has varied greatly. It is unclear if training is necessary for peers to provide effective support in improving health outcomes, or if peers on their own, by the nature of their own personal characteristics and backgrounds are able to offer effective support. Furthermore, as Thom et al. (2013) has pointed out, little is known about how the diabetes peer coaching results would apply to other chronic diseases, especially once they are extended into community settings. Finally, more research is needed to understand how peer characteristics influence the peer support relationship. Understanding how peer characteristics influence satisfaction and health improvement will improve how peers are matched and trained. Therefore, this study aimed to: examine the feasibility and preliminary outcomes of pairing peers, without training, in a community based weight management program and to explore factors influencing the peer relationship. The following research questions guided the study: 1) How does total weight-loss and weight-loss trajectory over three months differ between those paired with a peer and those who are not paired, and 2) What characteristics influence the perceived quality or effectiveness of the peer support?

\section{Method}

The current study implemented a nonequivalent group, quasi-experimental design. Participants were partnered with a peer (serving as the intervention), given brief instruction on their options for weekly interaction, and assessed at three months on a variety of psychosocial, 
behavioral, and anthropometric outcomes. A comparison group without a peer partner was used to assess differences in total weight-loss and weight-loss over time. Each group completed a baseline weight measurement and monthly weight measurement for three-months.

\section{Participants and Recruitment}

Participants were selected from the weight management program (WMP) run by the Public Employees' Insurance Agency (PEIA) in West Virginia. The PEIA WMP is a two-year program open to any state employee with PEIA insurance, or their families, who have either a BMI over 25 or a waist circumference over 35 inches for women, or over 40 inches for men. Participants pay a \$20 monthly co-payment to join the program, which gives them access to an eligible gym of their choosing, medical nutrition therapy with a registered dietician, fitness assessments with an exercise physiologist, personal training, and phone health-behavior coaching. As long as participants are active in the program and show progress they can remain in the program for two years. Participants had 60 community-based fitness centers throughout the state of WV to choose from. See Zizzi, Abildso, Henderson, \& Cobb, (2014) for further details on the PEIA weight management program. To participate in the current study, participants met eligibility requirements for the program and voluntarily agreed to participate in the peer support program.

From January to April 2018190 participants enrolled in the WMP and were contacted to inform about the optional peer support program and 40 were partnered with a peer. Of the 40 partnered, five participants were removed from final analysis. One pair was removed due to a partner dropping and failing to make contact, one pair was removed due to requesting new partner who was at the same fitness facility, a final pair requested a new partner due to incompatibility between peers, however one of the peers was able to be re-partnered. Of these 35 
participants four (two pairs) were removed after reviewing the survey data as they failed to report regular contact, leaving a final sample of 31 participants (which included one peer group of three who all requested to be partnered together, and one mixed gender pairing who also requested to be partnered). The final sample of 31 ( 28 female, 3 male) had a mean baseline BMI of $37.64(S D \pm 8.53)$ and a mean age of $41.29(S D \pm 11.54)$.

Comparison group. The PEIA WMP has been running for 10-years and weight-loss data has been collected on over three thousand participants. Data collected from January, 2015 to January, 2018 was used to create a comparison group derived from participants who had completed the program in the past, or those who were currently active. A true experimental design manipulating peer support was not possible due to the WMP's insurance company sponsorship, as the insurance company required all participants to have the opportunity to participate in the peer support program. Thus, to study how peer support in a community based WMP influenced weight-loss outcomes the researcher used a technique called propensity score matching to create a comparison group.

A propensity score represented the probability of a participant being in treatment on the basis of the baseline demographic variables (Randolph, Falbe, Manuel, \& Balloun, 2014; Rosenbaum \& Rubin, 1985). The propensity score was calculated by modeling multiple baseline demographic variables to create a single propensity score that equally balances demographic variables across the treated and non-treated groups. In this way the propensity score was used to minimize selection bias. Each participant is assigned a propensity score based on their baseline characteristics. This score is then used to create a comparison group.

In the current study participants were matched using the nearest neighbor matching method, with an exact match for participant gender. The approach first assigns a score to each 
participant within a study using covariates. It then randomly orders said participants within each group and pairs them with a counterpart, of the same gender, based on similar scores within a range. In this study, this range was defined by a caliper of 0.20 , or a similar score within $+/-$ 0.20. Both participants were then removed from the pool of possible matches and the next treatment participant was selected and matched in the same manner.

Propensity matching, by incorporating multiple covariates, attempts to control for bias that may exist due to treatment assignment; this increases confidence that any differences in weight-change from peer support were due to the treatment and not to error (d'Agostino, 1998). Participant baseline age and BMI were analyzed to calculate propensity scores for each participant. Participants were then randomly organized peer versus comparison group in order to build the comparison group. A participant in the peer group was randomly selected and the model matched a comparison participant of the same gender whose propensity score was within 0.20 of the peer participant. Upon matching, both participants were removed and the next peer participant was selected and matched in the same manner. This created two groups in which baseline BMI and age were equally balanced across groups.

\section{Procedure}

Upon enrollment in the WMP new participants were contacted via email or phone to inform them of the optional peer support program. Participants were told that PEIA is currently testing a new program that will pair members to provide support and accountability in reaching their goals. Requirements of participation will involve at-least one weekly contact with their peer, which can take place in-person, over the phone, via text, or email. In addition, participants were told they would be asked to fill out an additional survey at enrollment and each month 
during the three-month peer support evaluation period. (see Appendix B for recruitment and enrollment scripts).

New members who agreed to participate in the peer support program were partnered with another member who was also in the WMP. Participants were matched with a same gender peer, and effort was made to match within the same geographic area (e.g. same exercise facility), but if a lack of participants prevented pairing by location, then peers were paired with an available same gender participant at another location. In cases where participants requested to be partnered with a specific member in the program permission was obtained from the requested participant and they were then partnered.

Each member who elected to participate in the peer support program received a brief $(\sim 5$ minute) phone call to orient them to their role as a peer supporter (see Appendix B). Participants were asked to contact their peer a minimum of once per week either by phone, text, email, or inperson. As a peer supporter they instructed to provide encouragement, accountability, and share knowledge to help connect each other to available resources already in place within the program (e.g. registered dietician or health-behavior counselor). They were told not to provide any counseling, medical, nutrition, or exercise advice. Finally, each individual in a peer pairing was introduced via email (see Appendix B for email script).

\section{Measures}

Upon enrollment, participants in both the comparison and peer group completed the following baseline measures (see Appendix $\mathrm{C}$ for a table with a schedule for measures). The demographic information included: gender, health status, prior weight-loss attempts, marital status, family situation (e.g. mother, children at home), expectations for program, support for exercise and healthy eating, and a depression measure. Support for exercise and healthy eating 
was gathered through two self-report questions: 'how much could you count on those close to you for support and help if you wanted to eat a healthier diet/become more physically active?' Participants respond with 'not all', 'very little', 'somewhat', or 'a lot', responses were scored from one to four for each question, with a higher score indicating higher support. Depression was measured using the Center for Epidemiologic Studies Depression Scale (CES-D), which assessed depression through participant ratings of frequency of 20 different feelings or behaviors over the past week (see Appendix D; Radloff, 1977). Frequency was from scored zero (rarely or none of the time, less than 1 day in past week) to three (most or all of the time, 5-7 days in last week), with total scores of 16 or higher indicating risk for clinical depression. The scale was shown to have high internal consistency ( $\alpha=0.85-0.90)$ across three samples and validity was supported by correlations with self-report measures and clinical ratings of depression (Radloff, 1977).

Anthropometric data was collected at each participating fitness facility and entered at the site into a secure database. This database was then accessible to the staff at the PEIA WMP office located at West Virginia University (WVU). Each site collected participant weight, blood pressure, percent body fat, and waist circumference at enrollment, and throughout their program on a monthly basis.

Those who elected to participate in the peer support program were asked to complete four additional surveys online. These surveys were emailed to participants through an online survey management tool when partnered with their peer, and the end of each of the following threemonths. At enrollment and at completion of the three-month peer support program participants were sent a link to a survey to assess level of support with the 16-item Interpersonal Support Evaluation List-Short Form (ISEL; see Appendix E; Payne et al., 2012). In addition, at the completion of the first, second, and third month in the peer support program, participants were 
also asked to report the number of contacts they had with their peer in the past month, the mode(s) of contact used (e.g. phone, text, email, in-person), and to rate their level of satisfaction with the support they received on a slider from zero (not at all satisfied) to 100 (very satisfied). At the end of each of these surveys participants were reminded of the primary investigator's contact information in case they had any follow up questions or concerns.

The ISEL (S. Cohen, Mermelstein, Kamarck, \& Hoberman, 1985) was created based on the buffering hypothesis, which states that responses to stress are moderated, or buffered by the support we receive. From this hypothesis four subscales of support were created in the original ISEL: Tangible support assesses the perceived availability of material aid; Appraisal support assesses the perceived availability of someone to talk to about one's problems; Self-esteem support assesses the perceived availability of a positive comparison when comparing oneself to others; and Belonging support assesses the perceived availability of people one can do things with. Each subscale uses 10 -items that are rated on a four-point scale from definitely false to definitely true. A modified version of the scale was created by shortening the number of items per subscale from 10 to four (see appendix E; Payne et al., 2012). This updated scale was found to have strong internal reliability, $\alpha=0.83$, across a large probability sample, and moderate reliability for each of the four subscales $(\alpha=0.53-0.67)$. Further, construct validity was found through correlations with another measure of social support and negative correlations to health conditions hypothesized to increase in absence of support (e.g. depression, anxiety). In the current study, Cronbach's alphas indicated moderate reliability of the overall scale $(\alpha=0.74)$, and low reliability for the appraisal $(\alpha=0.22)$, tangible asset $(\alpha=0.23)$, belonging $(\alpha=0.41)$, and self-esteem $(\alpha=0.02)$ subscales.

\section{Data Analysis}


To answer research question one, "what effect does pairing peers have on total weightloss and weight-loss trajectory over three months compared to those who were not paired?", quantitative weight change data was analyzed using hierarchical linear modeling (HLM) software (Raudenbush, Bryk, \& Congdon, 2013). Model building was done in three steps. First, the best level one model was built to model weight change over the three months of study by comparing models with a linear and a quadratic time variable to the null model. The time variables were centered at three-months, so that the intercept of the level one model represented total weight-loss at three months. Second, control and the peer intervention variables were entered at level two. Control variables included each participant's propensity score, gender, initial BMI and age centered at the grand mean. These were the same variables used for Propensity Score Matching, but were entered again to control for additional variance after matching was completed. Participants who had a peer were dummy coded $(0=$ control, $1=$ peer $)$ and entered at level two in the model in order to measure the effect of peer support on total weight change and on weight change over time compared with the comparison group. Finally, each fitness facility was entered at level three in order to control for variance in outcomes based on differences in fitness facility location.

To answer research question two, "what characteristics influence the peer relationship?" descriptive statistics and effect sizes were calculated using Cohen's d and compared across different subgroups of peers. Peer subgroups included: those who requested a partner versus random partner, those who had an age difference greater than 10 years versus 10 years or less, and those with a BMI difference greater than 10 versus those with a BMI difference less than 10 . A cutoff point of 10 was created based on the data as this created roughly equal comparison groups. For any moderate or large effects in weight-loss between subgroups HLM was run to 
model weight loss and examine how the subgroup influenced total weight-loss and weightchange over time. Participant control variables (age, baseline BMI, and gender) and peer subgroup (dummy coded) were entered at level two.

Data cleaning. First, peer dyads that did not complete a follow up survey indicating regular contact between the two were removed from the analysis. Regular contact was defined as at least one contact each of the three months. Dyads for which at least one peer completed a follow up survey indicating contact was made were kept in the final sample. Finally, participant weight data was entered into database at fitness facilities and was downloaded for analysis. In the case of missing three-month weight data the previous weight entered was carried forward to capture weight change at three months.

\section{Results}

Mean number of contacts between peers per month was $7.86(S D \pm 8.15)$. After one month, 23 of $31(74 \%)$ participants reported being satisfied with their peer partner (satisfaction > $50)$, and after two and three months, respectively, 17 of $26(65 \%)$ and 15 of 23 respondents $(65 \%)$ reported still being satisfied. See Table 1 for full results. The mean total baseline ISEL score was $18.41(S D \pm 4.37)$, with participants increasing by a mean of $10.95(S D \pm 5.03)$ after three months with their peer (see Table 2). Both ISEL scores at baseline and three-months were low as compared with a normative value of 37 (Payne et al., 2012). Texting and email were the most commonly reported method of contact between peers, with phone and video chat the least frequent methods (see Table 3).

\section{Research Question One}


The following results focused on answering research question two: What effect does pairing peers have on total weight-loss and weight-loss trajectory over three months compared to those who were not paired?

Predictors of peer enrollment. Logistic regression was run with 166 participants who did not enroll in the peer program and 32 who enrolled. Age, gender, baseline depression score, baseline support for physical activity and diet, and baseline BMI centered at 25 were entered as predictor variables, with none of the variables being significant predictors of peer support enrollment. See Table 4 for logistic regression results.

Propensity score matching. Propensity score matching was run in SPSS using the PS Match program (Thoemmes, 2012). Participants were matched with an exact match for gender, propensity scores were calculated based on participant age and baseline BMI and matched using the nearest neighbor matching method with a 0.20 caliper. This process resulted in 30 of 31 peers being matched, for a total sample of 60 participants. See Figure 1 for distribution of propensity scores after matching which showed a good match between groups. Due to the small sample and high number of participants matched a sensitivity analysis could not be completed. See Table 5 for comparison of percent weight change, age, and baseline BMI between peer and matched sample. Three of 30 in the matched group and one out of 30 in the peer group dropped before completing three-months in the weight management program.

Modeling weight change. A graph of participant mean weight change per month showed a linear relationship over the three-months of study (see Figure 2). A linear model was the best fit for the data as compared with the null model $X^{2}(1, N=2)=48.8, p<0.01$, and adding a quadratic term to the model did not significantly improve model fit $X^{2}(1, N=2)=0.1, p>0.05$. $7.73 \%$ of variability in weight change was within participants at level one, $91.44 \%$ was between 
participants at level two, while only $0.83 \%$ of variance existed between fitness facilities at level three. The low variance at level three was partially due to the small sample size, which resulted in most fitness facilities having few participants. Due to the low variability and lack of participants in level three clusters (fitness facilities) the third level was removed and analysis of weight change was run with a two level model. The Intraclass Correlation Coefficient (ICC) of the two-level model was $93 \%$, indicating a majority of the variance existed at level-two between participants. A histogram of residual variance at level one revealed a normal distribution (see Figure 3), while histograms of residual variance of level two intercept (see Figure 4) and level two linear slope (see Figure 5) showed a slight positive skew.

The parameters for the final model can be found in Table 6 and the final full model with parameter interpretation can be found in Appendix F. After controlling for gender, age, baseline $\mathrm{BMI}$, and propensity score the intercept remained significant, $\beta_{00}=-4.78, p<0.01$, indicating that a female participant without peer support lost an average of 4.78 pounds at three months, holding all other variables at the grand mean. Being partnered with a peer did not have a significant effect on the intercept $\beta_{04}=1.14, p=0.52$. The positive value indicates that those partnered with a peer lost less weight overall, but this was not significant. After controlling for gender, age, baseline BMI, and propensity score the linear slope remained significant, $\beta_{10}=$ $1.62, p<0.01$, indicating that a female participant without peer support lost an average of 1.62 pounds each month, holding all other variables at constant. Again being partnered with a peer did not have a significant effect on linear weight change, $\beta_{14}=0.57, p=0.33$. The positive value indicates that those partnered with a peer had slower linear weight-loss each month, but this result was not significant.

\section{Research Question Two}


The following results focused on research question one: what characteristics influence the perceived quality or effectiveness of the peer support? Mean differences were explored using Cohen's d to calculate effect sizes instead of reporting statistical differences tests due to the small sample size. Effect sizes were interpreted as small for values from 0.2 but less than 0.5 , moderate from 0.5 but less than 0.8 , and large for values greater than 0.8 (J. Cohen, 1992). For clarity of effect the reporting of Cohen's d estimates are presented as absolute values with the direction of effect described in-text.

Partner request. Of the 31 peers, 13 requested to be partnered with a specific member in the program. Those who requested a partner were more satisfied $(d=1.72)$, contacted each other more frequently $(d=1.43)$, were older (Cohen's $d=0.37)$, reported higher total ISEL change $(d$ $=0.30)$, but lost less weight $(d=0.39)$. See Table 7 for full results across partner request.

BMI difference. When comparing BMI differences between peer pairings, the group of three was removed, leaving a final sample of 28, of whom 12 participants were partnered with a peer whose BMI was within 10 units while the remaining 16 participants were partnered with a peer whose BMI was greater than 10 units apart. Dyads with a BMI difference over 10 lost more weight $(d=0.96)$, had a higher baseline BMI $(d=0.86)$, were younger $(d=0.38)$, contacted each other less frequently $(d=0.29)$, and reported a smaller total ISEL change $(d=0.62)$. See Table 8 for full results across BMI differences.

To analyze the influence of BMI difference on weight change over time a two-level HLM model was built with only participants in the peer support condition. A linear model was again a better fit for data than the null model, $X^{2}(2, N=2)=119.67, p<0.01$. A histogram of the variance at level 1 (Figure 6) and level 2 intercept (Figure 7) and slope (Figure 8) showed a normal distribution. The ICC of the linear model was $90 \%$, indicating the majority of variance 
was between participants at level two. After controlling for participant gender and baseline BMI and age grand mean centered there was a significant effect for BMI difference, on both the intercept, $\beta_{03}=-6.37, p=0.02$, and slope, $\beta_{13}=-1.55, p=0.04$. Further, both the intercept, $\beta_{00}=-$ $0.52, p=0.79$, and slope $\beta_{10}=-0.28, p=0.60$ were not significant. These results indicate that after controlling for gender, BMI, and age only those with a peer dyad whose BMI difference was greater than 10 lost a statistically significant amount of weight. The final model predicted that a female, with BMI and age at the grand mean, and a BMI difference greater than 10 would lose an additional six pounds at three-months and an additional pound and half each month, as compared with a peer who was partnered with a peer within 10 BMI units. See Table 9 for full parameter results.

Age difference. When comparing age differences between peer pairings the group of three was removed, leaving a final sample of 28 , of whom 16 participants were partnered with a peer whose age was within 10 years while the remaining 12 participants were partnered with a peer whose age was greater than 10 years apart. Those with an age difference under 10 years contacted each other more frequently $(d=0.97)$, but these groups were not different on baseline BMI $(d=0.06)$, percent weight-loss $(d=0.17)$, mean satisfaction $(d=0.11)$, or total ISEL change $(d=0.01)$. See Table 10 for full results across age differences.

Correlations. Pearson correlations between percent weight change at month three and participant baseline BMI, age, mean visits to the fitness facility per month, mean monthly contact between peers, mean three month satisfaction, BMI difference, age difference, ISEL change overall, and ISEL subscale change were all non-significant. However there were several small effects worth noting, including BMI difference $(r=-0.31)$, mean contacts per month $(r=$ 
$.28)$, age difference $(r=-0.20)$, and total ISEL change $(r=0.22)$ showed a small effect. See Table 11 for full results.

\section{Discussion}

Overall, the training of peers has varied greatly in focus, intensity, and duration across the diabetes and weight-loss research (Tang et al., 2011), and it remains unclear what training, if any, is necessary for peers to provide effective support. Thus, the purpose of this study was to evaluate the feasibility and preliminary outcomes of participants in a community based weight management program who provided support to a peer without receiving any training. First, preliminary outcomes of the three-month study period are presented, followed by takeaways from the peer program, and finally, the role of moderators is discussed.

\section{Change in Weight-loss}

Prior research has shown that weight-loss follows a linear path in the initial months in a program, then slows as the program progresses (Ingels, Misra, Stewart, Lucke-Wold, \& Shawley-Brzoska, 2017). The short duration of the present study likely explains why a quadratic term did not improve model fit, as participants were still early in their program and thus weight maintained a linear trajectory. Overall, the model predicted weight-loss of almost five pounds, with participants losing just over a pound and half each month. While peer support did not have a significant effect on total weight-loss or weight-loss over time there are several factors to consider when interpreting these results.

Unlike two meta-analyses of peer support research in diabetes management (Qi et al., 2015; Zhang et al., 2016) and two studies of peer support interventions in weight management (Leahey et al., 2016; Leahey \& Wing, 2013), which focused on studies where the peer component was the primary intervention, the current study evaluated the impact of optional peer 
support as an addition to a weight management program. The peer support in the current study may not have provided enough impact over the three-month study period to show differences in weight outcomes, as it was not the primary intervention. In addition, peer support was typically delivered via telephone contact among peers in diabetes programs (Heisler, Vijan, Makki, \& Piette, 2010; Qi et al., 2015; Zhang et al., 2016), and peers who used email contact to support each other in the peer weight-loss study received training focused on how to provide support and strategies to promote behavioral weight-loss (Leahey \& Wing, 2013). Participants in the current study preferred contact via email or text message, and thus they may have needed additional training on how to effectively deliver support. It is also possible that this form of text communication is less effective in supporting weight loss efforts than phone-based supports.

The supplemental peer support program may have impacted participants beyond weight loss, such as improving adherence to the weight management program long-term, which may improve outcomes over longer periods. Prior research studying the influence of dietary tracking on weight-loss found that adherent trackers lost more weight and their weight-loss trajectory differed over the 12-month program, but this difference was not noticeable during the initial months in the program (Ingels et al., 2017). As the program progressed, weight-loss slowed for low adherent trackers, while high adherent trackers continued to lose weight at the same rate. A systematic review reported programs including a social support component improved participant ability to maintain weight-losses at 12-months (Greaves et al., 2011). The long-term benefits of social support may improve outcomes through better adherence to program objectives, such as continuing to visit the gym, or meeting with personal trainers and dieticians. Research of medical adherence provides support for this theory, as a review of 122 studies found that multiple forms of social support increased participant adherence to medical treatment (DiMatteo, 2004). It is 
possible that in the current study, the support received from peers will lead to improved adherence, which in turn would improve weight-loss outcomes over the course of the two-year program. Preliminary results of participant drops in the current study found that one of 30 in the peer program dropped before three-months, while three out of 30 dropped in the comparison group. Over the course of two years it is possible that fewer participants drop when partnered with a peer, which could lead to improved weight-loss outcomes.

The self-selection bias of those enrolling in the optional peer support program may have influenced the ability of the model to detect a difference in outcomes. As discussed previously, the peer program was offered in addition to a structured weight management program, which, along with the low baseline scores on the ISEL, may indicate that those who enrolled in the peer support program felt they needed additional support in reaching their weight-loss goals. The ISEL was unable to be used in the propensity score analysis as only those in the peer program completed this measure. The propensity score analysis created a good match based on the available covariates, but it is possible that another unmeasured covariate would have captured this need for additional support, further minimizing the self-selection bias, and improving the ability of the model to detect a difference in weight loss outcomes.

Finally, it is important to consider the differences between weight and diabetes management programs. First, diabetes management programs have standard curriculums to follow which include regular checking of blood sugar, managing insulin, diet, and physical activity (Aziz, Absetz, Oldroyd, Pronk, \& Oldenburg, 2015; Knowler et al., 2002). The strategies for weight management can vary widely from using prepackaged foods/shakes to focusing on eating smaller portions to increasing physical activity (Miller, Koceja, \& Hamilton, 1997; Ramage, Farmer, Apps Eccles, \& McCargar, 2013; Tsai, 2005). In addition, the consequences 
for failing to manage blood sugar are more immediate and severe than failing to lose weight, such as immediate decreases in energy, coma, and limb amputation (International Diabetes Federation, n.d.). Within diabetes management a peer can help by sharing their own experience with insulin injections, monitoring blood sugar, and sharing strategies for managing blood sugar levels, while for weight-loss a strategy that works for one person (e.g. increasing physical activity) may not result in weight-loss for another. Thus, sharing personal experience may not have the same effect in weight management. Given these differences, further research is needed to better understand how to effectively incorporate peer support in weight management and what training peers need in order to deliver effective support.

\section{Peer Support Takeaways}

The current study adds to our understanding of peer support in weight management in several ways. Overall, 31 of 40 participants completed follow-up surveys and the reported number of average contacts per month was high as compared with a meta-analysis of peer support interventions in diabetes management, which classified high contact as more than two contacts per month (Qi et al., 2015). Further, over 70-percent of participants were satisfied with their peer after month one, with $65 \%$ remaining satisfied at months two and three. These results suggest that the peers, without training, were satisfied with the support they received and were motivated to maintain regular contact with their partner. Prior self-report data from peer support in diabetes reported a similar percent of participants being satisfied with peer support (Heisler \& Piette, 2005; Safford et al., 2015). Further, exploration of contact mode used by peers revealed that text messaging and email were the most common ways peers connected. Prior research reported that participants enjoyed the convenience of phone calls (Dutton et al., 2015) and the anonymity that communicating via phone provided (Heisler \& Piette, 2005). It is unclear why 
participants in the current study chose to predominantly communicate by text and email. Further mixed methods or qualitative research may be needed to provide context to this finding.

Participant self-selection into the optional peer program created an opportunity to explore characteristics of those who enrolled. Interestingly, age, gender, baseline BMI, baseline depression score, and participant support did not significantly predict who enrolled. To date, there has been a lack of research focused on who seeks out peer support. A better understanding of who seeks out peer support can inform how peer programs are structured and the type of peer training that may be needed. While none of the results were significant, several factors are worth considering. First, a low number of males enrolled in the peer program, making it difficult to determine the effect of gender on peer support enrollment. This low enrollment is not surprising in the current weight management program that typically maintains a large majority $(\sim 85 \%)$ of women. Men may also be less likely to seek out peer support. Future studies, with larger samples or a mixed design can focus on understanding how perceptions of peer support differ between males and females. Second, the support score used in the logistic regression was a rough estimate using responses from two four-point scales. Future research is needed to explore the role of support using a validated support scale.

Prior meta-analyses have shown the benefit of social support in both weight-loss and weight maintenance (Greaves et al., 2011; Huisman et al., 2009), while qualitative research has highlighted the important role of support from staff (Abildso, Zizzi, Gilleland, Thomas, \& Bonner, 2010) and peers (Wang et al., 2015) in achieving weight-loss goals. Social support has been theorized to impact health by helping to cope with challenges and embracing opportunities for growth and change (Feeney \& Collins, 2015). In the current study, self-reported results from the ISEL revealed low baseline scores on the total scale, as scores were over 50-percent lower 
than scores reported across a normative sample of over 14,000 (Payne et al., 2012). While overall ISEL scores increased by almost 11-points (a 59\% increase) at the third month, these scores were still 10 points lower than the scores in the Payne et al. (2012) sample. This increase is promising as it shows the those in the peer program felt more support after just three-months, yet this increase is likely due to a combination of receiving support from a peer and from program staff. Future research can address this issue by having a control group to explore how change in social support influences behavioral and health outcomes. A better understanding of how peers provide support can inform the structure, content, and training that is needed. For example, using the Feeney and Collins theory (2015) as a framework, research of peers in weight-loss can focus on understanding whether support in the face of challenges, support in embracing opportunities for growth, or a combination of the two lead to better results. Finally, the ISEL subscale results must be interpreted with caution as prior research has shown moderate reliability (Payne et al., 2012) and the current study found the subscales were not reliable. Further research is needed to confirm reliability of each subscale, especially within a sample focused on weight management.

\section{Moderators of Peer Support}

Those who requested a partner reported higher satisfaction, larger improvements in perceived support, and more contact, but lost less weight at three months. Some research has suggested that receiving support from a self-selected person does not improve weight-loss outcomes as compared with a group without a formal social support component (Rieger, Treasure, Murray, \& Caterson, 2017). It is possible that weight-loss outcomes are improved most by being partnered with someone outside of one's current social sphere. Participants may benefit from a fresh perspective or being pushed in a way that someone who knows them already may be 
unwilling or unable to do. Further, Rieger et al. (2017) found that satisfaction with support from a participant-selected individual decreased over a 12-month study. In the present study, satisfaction also decreased slightly over the three-months, but was higher each month for those with a self-selected peer. One key difference may be that in the present study self-selected peers were also working to lose weight in the same program, whereas in the Rieger et al. (2017) study the selected support partners were not attempting to lose weight in the same program, but were just there to provide support. The lack of a true peer going through the same struggles may have influenced participant satisfaction in the Rieger study, as prior research has shown that participants appreciate receiving support from someone facing the same challenges as them (Heisler \& Piette, 2005). The data from the current study provides some support to this idea.

Peer dyads with closer BMIs reported a higher change in overall support, which suggests that participants may experience support in different ways depending on similarity in BMI. Participants with similar BMI may have been better able to understand each other's struggles and provide more effective support. Yet, despite these differences in support weight-loss was negligible for those with closer BMI's while those whose BMI's differed by over 10 units lost close to two-percent of their body weight by three months. After modeling weight change over time and accounting for participant characteristics, total weight-loss and weight-loss over time was significantly greater for participants whose BMIs differed by over 10 units. These results suggest that peers with closer BMIs reported a greater increase in support, but lost significantly less weight than those whose BMIs differed by over 10 units. Future research is needed to understand how these subgroups differed in their interactions.

The influence of BMI similarity among peers has not received much research attention. One study of BMI differences among peer coaches in diabetes found that coach BMI was 
connected with better outcomes, but in conflicting directions as lower BMI was associated with better outcomes for coaches with a BMI under 35, while a higher BMI was associated with improved outcomes for coaches with a BMI over 35 (Rogers et al., 2014). The mixed findings of Rogers et al., coupled with the current study's increase in weight-loss for those with a BMI difference over 10, warrants further study to determine how BMI similarity influences weightloss.

Finally, peers who were closer in age reported over twice as many contacts each month, but reported similar satisfaction, improvements in support, and weight-loss. The higher number of contacts among dyads whose age was closer may be due to communication preferences, as those closer in age may both prefer the same mode of contact (e.g. texting or phone). The results of the current study support prior findings which did not find an effect for similarity in age between peer coaches and their patients working to control their diabetes (Rogers et al., 2014). Further research is needed to understand how age impacts peers preferences for communication and how to utilize communication preferences when developing peer support programs.

These preliminary results show how requesting a partner, BMI, and age impact satisfaction, social support change, and weight outcomes for those partnered with a peer. The differences in support, satisfaction, average number of contacts, and weight change warrant further study with larger samples that would enable regression analyses to better understand how these variables influence outcomes and satisfaction with peer support. Peers, based on their characteristics, will bring different strengths and weaknesses to the peer support relationship. Understanding how these characteristics interact will improve the training and structure of peer support programs. For researchers and health professionals implementing a peer support program 
understanding how to effectively partner peers, how peer characteristics influence the relationship, and what training is necessary will improve the effectiveness of peer support.

\section{Limitations}

This study was not without limitations. First, participant self-selection into the peer support relationship could have resulted in self-selection bias that influenced the weight change results. It is possible that those who selected to work with a peer differed on some unknown or unmeasured psychological or physiological variable that impacted the results. While propensity score matching aimed to address this limitation by using covariates to minimize self-selection bias, it is inherently limited by the quality of the included covariates, and there is always the possibility that a missing covariate is a vital predictor. In addition, the three-month time period may have been too short for weight loss or behavioral results to emerge. Further, differences in ISEL are limited by lack of comparison group and the low reliability of subscales. Finally, exploration of moderators of peer support is limited by a small sample size.

\section{Future Directions}

Future studies can address these limitations in a variety of ways. First, a larger sample with a randomized control group and a longer follow-up would improve our understanding of peer support on weight-loss outcomes. Taking a mixed method approach to understanding how support differed between more and less successful peers would help understand how peers might need to be trained. Second, the differences in partner request, age, and BMI can be explored by conducting interviews with participants to understand how participants supported each other and areas where they needed additional support and training to improve outcomes. Questions can focus on how peers experienced support, what contributed to the high or low number of contacts, what influenced their rating of satisfaction, and how the peer helped (or did not) with their 
weight-loss goals. Finally, more research is needed to identify how to best match participants in a peer program. This study began this exploration by looking at age and BMI, along with requesting a partner versus a random partner. Future studies can build upon these results by creating groups to explicitly create matches based on BMI and/or age. For example, a study could create two groups by matching with a peer who is in the same baseline BMI category (e.g. both overweight or both class I obese) with a group who are partnered in different categories. The same process can be taken with age, ethnicity, personality characteristics, and mood measures (e.g. depression, anxiety) to determine if a certain mix of characteristics lead to improved satisfaction, support, and health improvements.

\section{Conclusion}

Overall, the results from the present study point to the feasibility for peers to provide support in an ongoing weight management program. Despite a lack of weight-loss at three months, a majority of peers were satisfied with peer support, contacted each other regularly, and reported increased support. The results suggest that intensive training may not be necessary for peers to provide impactful support in weight management. Though not surprising, it is important to note that the participants who self-selected into the current study reported low scores at the onset of the program. Thus, including a pre-screening tool for social support and offering services to those at the low end of the spectrum seems like a good initial approach. 


\section{References}

Abildso, C. G., Zizzi, S., Gilleland, D., Thomas, J., \& Bonner, D. (2010). A mixed methods evaluation of a 12-week insurance-sponsored weight management program incorporating cognitive-behavioral counseling. Journal of Mixed Methods Research, 4(4), 278-294. https://doi.org/10.1177/1558689810376949

Aziz, Z., Absetz, P., Oldroyd, J., Pronk, N. P., \& Oldenburg, B. (2015). A systematic review of real-world diabetes prevention programs: Learnings from the last 15 years. Implementation Science, 10, 1-17. https://doi.org/10.1186/s13012-015-0354-6

Baumann, L., Frederick, N., Betty, N., Jospehine, E., \& Agatha, N. (2015). A demonstration of peer support for Ugandan adults with type 2 diabetes. International Journal of Behavioral Medicine, 22(3), 374-383. https://doi.org/10.1007/s12529-014-9412-8

Chan, J. C. N., Sui, Y., Oldenburg, B., Zhang, Y., Chung, H., Goggins, W., ... Fisher, E. (2014). Effects of telephone-based peer support in patients with type 2 diabetes mellitus receiving integrated care: A randomized clinical trial. JAMA Internal Medicine, 174(6), 972-981. https://doi.org/10.1001/jamainternmed.2014.655

Christakis, N. A., \& Fowler, J. H. (2007). The spread of obesity in a large social network over 32 years. New England Journal of Medicine, 357(4), 370-379. https://doi.org/10.1056/NEJMsa066082

Cohen, J. (1992). A power primer. Psychological Bulletin, 112(1), 155.

Cohen, S., Mermelstein, R., Kamarck, T., \& Hoberman, H. M. (1985). Measuring the functional components of social support. In I. G. Sarason \& B. R. Sarason (Eds.), Social Support: Theory, Research and Applications (pp. 73-94). The Hague, The Netherlands: Martinus Nijhoff. https://doi.org/10.1007/978-94-009-5115-0_5 
d'Agostino, R. B. (1998). Tutorial in biostatistics: Propensity Score methods for bias reduction in the comparison of a treatment to a non-randomized control group. Statistics in Medicine, 17(19), 2265-2281.

Dennis, C.-L. (2003). Peer support within a health care context: A concept analysis. International Journal of Nursing Studies, 40(3), 321-332. https://doi.org/10.1016/S00207489(02)00092-5

DiMatteo, M. R. (2004). Social support and patient adherence to medical treatment: A metaanalysis. Health Psychology, 23(2), 207-218.

Dunkel Schetter, C. (2017). Moving research on health and close relationships forward-a challenge and an obligation: Introduction to the special issue. American Psychologist, 72(6), 511-516. https://doi.org/10.1037/amp0000158

Dutton, G. R., Phillips, J. M., Kukkamalla, M., Cherrington, A. L., \& Safford, M. M. (2015). Pilot study evaluating the feasibility and initial outcomes of a primary care weight loss intervention with peer coaches. The Diabetes Educator, 41(3), 361-368. https://doi.org/10.1177/0145721715575356

Feeney, B. C., \& Collins, N. L. (2015). A new look at social support: A theoretical perspective on thriving through relationships. Personality and Social Psychology Review, 19(2), 113147. https://doi.org/10.1177/1088868314544222

Goldman, M. L., Ghorob, A., Hessler, D., Yamamoto, R., Thom, D. H., \& Bodenheimer, T. (2015). Are low-income peer health coaches able to master and utilize evidence-based health coaching? Annals of Family Medicine, 13, 36-41. https://doi.org/10.1370/afm.1756 
Greaves, C. J., Sheppard, K. E., Abraham, C., Hardeman, W., Roden, M., Evans, P. H., \& Schwarz, P. (2011). Systematic review of reviews of intervention components associated with increased effectiveness in dietary and physical activity interventions. BMC Public Health, 11(1), 119-130. https://doi.org/10.1186/1471-2458-11-119

Heisler, M. (2010). Different models to mobilize peer support to improve diabetes selfmanagement and clinical outcomes: Evidence, logistics, evaluation considerations and needs for future research. Family Practice, 27, 23-32. https://doi.org/10.1093/fampra/cmp003

Heisler, M., \& Piette, J. D. (2005). "I help you, and you help me": Facilitated telephone peer support among patients with diabetes. Diabetes Educator, 31(6), 869-879.

Heisler, M., Vijan, S., Makki, F., \& Piette, J. D. (2010). Diabetes control with reciprocal peer support versus nurse care management: A randomized trial. Annals of Internal Medicine, 153(8), 507-515. https://doi.org/10.7326/0003-4819-153-8-201010190-00007

Huisman, S. D., Gucht, V. D., Dusseldorp, E., \& Maes, S. (2009). The effect of weight reduction interventions for persons with Type 2 Diabetes: A meta-analysis from a self-regulation perspective. The Diabetes Educator, 35(5), 818-835.

https://doi.org/10.1177/0145721709340929

Hwang, K. O., Ottenbacher, A. J., Green, A. P., Cannon-Diehl, M. R., Richardson, O., Bernstam, E. V., \& Thomas, E. J. (2010). Social support in an Internet weight loss community. International Journal of Medical Informatics, 79(1), 5-13. https://doi.org/10.1016/j.ijmedinf.2009.10.003

Ingels, J. S., Misra, R., Stewart, J., Lucke-Wold, B., \& Shawley-Brzoska, S. (2017). The effect of adherence to dietary tracking on weight loss: Using HLM to model weight loss over 
time. Journal of Diabetes Research, 2017. Retrieved from https://www.hindawi.com/journals/jdr/2017/6951495/abs/

International Diabetes Federation. (n.d.). Complications of diabetes. Retrieved October 17, 2016, from http://www.idf.org/complications-diabetes

Knowler, W. C., Barrett-Connor, E., Fowler, S. E., Hamman, R. E., Lachin, J. M., Walker, E. A., \& Nathan, D. M. (2002). Reduction in the incidence of type 2 diabetes with lifestyle intervention or Metformin. New England Journal of Medicine, 346(6), 393-403. https://doi.org/10.1056/NEJMoa012512

Kulik, N., Ennett, S. T., Ward, D. S., Bowling, J. M., Fisher, E. B., \& Tate, D. F. (2015). Brief report: A randomized controlled trial examining peer support and behavioral weight loss treatment. Journal of Adolescence, 44, 117-123. https://doi.org/10.1016/j.adolescence.2015.07.010

Leahey, T. M., Fava, J. L., Seiden, A., Fernandes, D., Doyle, C., Kent, K., ... Wing, R. R. (2016). A randomized controlled trial testing an internet delivered cost-benefit approach to weight loss maintenance. Preventive Medicine, 92, 51-57. https://doi.org/10.1016/j.ypmed.2016.04.013

Leahey, T. M., \& Wing, R. R. (2013). A randomized controlled pilot study testing three types of health coaches for obesity treatment: Professional, peer, and mentor. Obesity, 21(5), 928934. https://doi.org/10.1002/oby.20271

Martire, L. M., \& Helgeson, V. S. (2017). Close relationships and the management of chronic illness: Associations and interventions. American Psychologist, 72(6), 601-612. https://doi.org/10.1037/amp0000066 
Miller, W. C., Koceja, D. M., \& Hamilton, E. J. (1997). A meta-analysis of the past 25 years of weight loss research using diet, exercise or diet plus exercise intervention. International Journal of Obesity \& Related Metabolic Disorders, 21(10). Retrieved from http://www.optimalhealthpartner.com/A_Archive/MetaAnalysis_Miller.pdf

Moskowitz, D., Thom, D. H., Hessler, D., Ghorob, A., \& Bodenheimer, T. (2013). Peer coaching to improve diabetes self-management: Which patients benefit most? Journal of General Internal Medicine, 28(7), 938-942. https://doi.org/10.1007/s11606-013-2367-7

Payne, T. J., Andrew, M., Butler, K. R., Wyatt, S. B., Dubbert, P. M., \& Mosley, T. H. (2012). Psychometric evaluation of the Interpersonal Support Evaluation List-Short Form in the ARIC study cohort. Sage Open, 2(3), 2158244012461923.

Qi, L., Liu, Q., Qi, X., Wu, N., Tang, W., \& Xiong, H. (2015). Effectiveness of peer support for improving glycaemic control in patients with type 2 diabetes: A meta-analysis of randomized controlled trials. BMC Public Health, 15(1), 1-11. https://doi.org/10.1186/s12889-015-1798-y

Radloff, L. S. (1977). The CES-D scale: A self-report depression scale for research in the general population. Applied Psychological Measurement, 1(3), 385-401.

Ramage, S., Farmer, A., Apps Eccles, K., \& McCargar, L. (2013). Healthy strategies for successful weight loss and weight maintenance: A systematic review. Applied Physiology, Nutrition, and Metabolism, 39(1), 1-20.

Randolph, J. J., Falbe, K., Manuel, A. K., \& Balloun, J. L. (2014). A step-by-step guide to Propensity Score Matching in R. Practical Assessment, Research \& Evaluation, 19(18), $1-6$. 
Raudenbush, S. W., Bryk, A. S., \& Congdon, R. (2013). HLM 7.01 for Windows (Version 7.01). Skokie, IL: Scientific Software International, Inc.

Rieger, E., Treasure, J., Murray, K., \& Caterson, I. (2017). The use of support people to improve the weight-related and psychological outcomes of adults with obesity: A randomised controlled trial. Behaviour Research and Therapy, 94, 48-59.

Rogers, E. A., Hessler, D. M., Bodenheimer, T. S., Ghorob, A., Vittinghoff, E., \& Thom, D. H. (2014). Diabetes peer coaching: Do "better patients" make better coaches? The Diabetes Educator, 40(1), 107-115. https://doi.org/10.1177/0145721713513178

Rosenbaum, P. R., \& Rubin, D. B. (1985). Constructing a control group using multivariate matched sampling methods that incorporate the propensity score. The American Statistician, 39(1), 33-38.

Safford, M. M., Andreae, S., Cherrington, A. L., Martin, M. Y., Halanych, J., Lewis, M., ... Richman, J. S. (2015). Peer coaches to improve diabetes outcomes in rural Alabama: A cluster randomized trial. Annals of Family Medicine, 13, S18-S26. https://doi.org/10.1370/afm.1798

Tang, T. S., Ayala, G. X., Cherrington, A., \& Rana, G. (2011). A review of volunteer-based peer support interventions in diabetes. Diabetes Spectrum, 24(2), 85-98.

Thoemmes, F. (2012). Propensity score matching in SPSS. Cornell University. Retrieved from https://arxiv.org/abs/1201.6385

Thom, D. H., Ghorob, A., Hessler, D., De Vore, D., Chen, E., \& Bodenheimer, T. A. (2013). Impact of peer health coaching on glycemic control in low-income patients with diabetes: A randomized controlled trial. Annals of Family Medicine, 11(2), 137-144. https://doi.org/10.1370/afm.1443 
Tsai, A. G. (2005). Systematic review: An evaluation of major commercial weight loss programs in the United States. Annals of Internal Medicine, 142(1), 56. https://doi.org/10.7326/0003-4819-142-1-200501040-00012

Wang, J., Shih, P. C., \& Carroll, J. M. (2015). Life after weight loss: Design implications for community-based long-term weight management. Computer Supported Cooperative Work (CSCW), 24(4), 353-384. https://doi.org/10.1007/s10606-015-9226-5

Wilson, W., \& Pratt, C. (1987). The impact of diabetes education and peer support upon weight and glycemic control of elderly persons with Noninsulin Dependent Diabetes Mellitus (NIDDM). American Journal of Public Health, 77(5), 634-635.

Wing, R. R., \& Jeffery, R. W. (1999). Benefits of recruiting participants with friends and increasing social support for weight loss and maintenance. Journal of Consulting and Clinical Psychology, 67(1), 132.

Yin, J., Wong, R., Au, S., Chung, H., Lau, M., Lin, L., ... Chan, J. C. N. (2015). Effects of providing peer support on diabetes management in people with type 2 diabetes. The Annals of Family Medicine, 13(Suppl 1), S42-S49. https://doi.org/10.1370/afm.1853

Zhang, X., Yang, S., Sun, K., Fisher, E. B., \& Sun, X. (2016). How to achieve better effect of peer support among adults with type 2 diabetes: A meta-analysis of randomized clinical trials. Patient Education and Counseling, 99(2), 186-197.

https://doi.org/10.1016/j.pec.2015.09.006

Zizzi, S., Abildso, C., Henderson, N., \& Cobb, K. (2014). The West Virginia PEIA Weight Management Program: An Innovative approach to obesity prevention and treatment in Appalachian communities. In V. M. Brennan, S. K. Kumanyika, R. E. Zambrana, V. M. Brennan, S. K. Kumanyika, \& R. E. Zambrana (Eds.), Obesity interventions in 
underserved communities: Evidence and directions. (pp. 282-289). Baltimore, MD, US:

Johns Hopkins University Press. 
Table 1.

Descriptive Statistics for Peer Support.

\begin{tabular}{lccc}
\hline & $\mathrm{n}$ & Mean & SD \\
\hline Baseline BMI & 31 & 37.64 & 8.53 \\
Baseline Waist & 30 & 42.24 & 7.02 \\
Age & 31 & 41.29 & 11.54 \\
\% 3-Month Weight Change & 31 & -1.57 & 2.50 \\
Mean Visits/Month & 30 & 8.75 & 1.75 \\
Month 1 Contacts & 30 & 8.57 & 8.22 \\
Month 2 Contacts & 27 & 7.67 & 8.60 \\
Month 3 Contacts & 22 & 7.14 & 9.49 \\
Mean Contacts/Month & 30 & 7.86 & 8.15 \\
Satisfaction Month 1 & 30 & 68.30 & 31.40 \\
Satisfaction Month 2 & 26 & 66.92 & 33.36 \\
Satisfaction Month 3 & 22 & 62.82 & 31.98 \\
Mean Satisfaction & 30 & 66.56 & 30.38 \\
& & & \\
\hline
\end{tabular}


Table 2.

ISEL Statistics.

\begin{tabular}{lcc}
\hline & Mean Baseline (n=27) & Mean 3-Month Change (n=20) \\
\hline ISEL Total & $18.41(4.37)$ & $10.95(5.03)$ \\
Appraisal Support & $3.89(1.45)$ & $2.40(1.79)$ \\
Tangible Asset Support & $3.85(1.32)$ & $4.95(1.67)$ \\
Belonging Support & $4.33(1.71)$ & $1.90(1.71)$ \\
Self-Esteem Support & $6.33(1.14)$ & $1.70(1.66)$ \\
\hline
\end{tabular}

$\overline{\text { Note. Standard Deviation in parentheses. ISEL total scores can range from 0-48 and each }}$ subscale from 0-12. Appraisal support: the perceived availability of someone to discuss issues of personal importance. Tangible asset support: perceived availability of material aid. Belonging support: the perceived availability of others to interact with socially. Self-esteem support: the perceived availability of others with whom one compares with favorably. 
Table 3.

Frequency of Different Modes of Contact by Month.

\begin{tabular}{lcccccc}
\hline & Mean Contact/Mo. & Text & Phone & Email & In-Person & Video Call \\
\hline Month 1 $(\mathrm{n}=30)$ & $8.57(8.22)$ & 19 & 6 & 15 & 11 & 0 \\
Month 2 $(\mathrm{n}=27)$ & $7.67(8.60)$ & 15 & 3 & 13 & 9 & 0 \\
Month 3 $(\mathrm{n}=22)$ & $7.14(9.49)$ & 10 & 4 & 5 & 8 & 0 \\
\hline
\end{tabular}

Note. Mean Contacts per Month show SD in parentheses. 
Table 4.

Logistic Regression Output Predicting Enrollees in Peer Support Program.

\begin{tabular}{lcccccc}
\hline & B & S.E. & Wald & df & Sig. & Exp(B) \\
\hline Age & -0.03 & 0.02 & 3.13 & 1.00 & 0.08 & 0.97 \\
Gender & 0.50 & 0.54 & 0.86 & 1.00 & 0.35 & 1.65 \\
CESD & -0.07 & 0.04 & 3.24 & 1.00 & 0.07 & 0.93 \\
Support Diet \& PA & 0.12 & 0.17 & 0.47 & 1.00 & 0.49 & 1.12 \\
Start BMI Centered at 25 & 0.02 & 0.02 & 0.61 & 1.00 & 0.44 & 1.02 \\
Constant & -0.58 & 1.86 & 0.10 & 1.00 & 0.76 & 0.56
\end{tabular}

Note. Center for Epidemiologic Studies Depression Scale (CESD). Support for Diet \& Physical Activity self-report scores range from 0-8. 
Table 5.

Descriptive Comparison between Matched Propensity Score (PS) and Peer Groups.

\begin{tabular}{lcc}
\hline & Matched PS & Peer \\
\hline 3-Month \% Weight Change & $-2.39(3.74)$ & $-1.50(2.51)$ \\
Age & $43.40(12.02)$ & $41.90(11.22)$ \\
Start BMI & $39.04(11.16)$ & $37.17(8.25)$ \\
Mean Visits/Month & $8.77(3.57)$ & $8.76(1.77)$
\end{tabular}

Note. Standard Deviation in parentheses. 
Table 6.

Final HLM Model Parameters for Full Matched Sample.

Fixed Effect Coefficient Standard Error $p$-value

For Intercept, $\pi_{0}$

$\begin{array}{rccc}\text { Intercept, } \beta_{00} & -4.78 & 1.27 & <0.01 \\ \text { Age }^{*}, \beta_{01} & 0.22 & 0.37 & 0.57 \\ \text { Start BMI*, } \beta_{02} & -0.11 & 0.28 & 0.69 \\ \text { Gender, } \beta_{03} & -1.09 & 4.07 & 0.79 \\ \text { Peer Support, } \beta_{04} & 1.14 & 1.76 & 0.52 \\ \text { PS*, } \beta_{05} & 412.63 & 852.07 & 0.63\end{array}$

For Linear Slope, $\pi_{l}$

$\begin{array}{rccc}\text { Intercept, } \beta_{10} & -1.62 & 0.42 & <0.01 \\ \text { Age }^{*}, \beta_{11} & 0.08 & 0.12 & 0.51 \\ \text { Start BMI }{ }^{*}, \beta_{12} & -0.05 & 0.09 & 0.59 \\ \text { Gender, } \beta_{13} & -0.75 & 1.35 & 0.58 \\ \text { Peer Support, } \beta_{14} & 0.57 & 0.58 & 0.34 \\ \text { PS }^{*}, \beta_{15} & 166.99 & 282.23 & 0.56\end{array}$

*Indicates variable was centered at the grand mean. 
Table 7.

Means, Standard Deviations, and Effect Size by Peer Partner Request.

\begin{tabular}{|c|c|c|c|c|c|c|c|}
\hline \multirow[t]{2}{*}{ Partner Request } & \multicolumn{3}{|c|}{ No } & \multicolumn{3}{|c|}{ Yes } & \multirow[b]{2}{*}{ Cohen's d } \\
\hline & $\mathrm{n}$ & Mean & $\mathrm{SD}$ & $\mathrm{n}$ & Mean & $\mathrm{SD}$ & \\
\hline Age & 18 & 39.56 & 13.39 & 13 & 43.69 & 8.26 & 0.37 \\
\hline Baseline BMI & 18 & 38.11 & 8.36 & 13 & 36.99 & 9.05 & 0.13 \\
\hline \% 3-Month Weight Change & 18 & -1.97 & 2.75 & 13 & -1.02 & 2.08 & 0.39 \\
\hline Mean Visits/Month & 18 & 8.78 & 2.04 & 12 & 8.71 & 1.26 & 0.04 \\
\hline Mean Contacts/Month & 18 & 3.84 & 4.54 & 12 & 13.88 & 8.79 & 1.43 \\
\hline Mean Satisfaction & 18 & 51.33 & 30.25 & 12 & 89.40 & 8.37 & 1.72 \\
\hline ISEL Change & 12 & 10.33 & 5.09 & 8 & 11.88 & 5.14 & 0.30 \\
\hline Appraisal Support Change & 12 & 2.00 & 1.81 & 8 & 3.00 & 1.69 & 0.57 \\
\hline Tangible Asset Support Change & 12 & 4.83 & 1.75 & 8 & 5.13 & 1.64 & 0.17 \\
\hline Belonging Support Change & 12 & 1.75 & 1.96 & 8 & 2.13 & 1.36 & 0.22 \\
\hline Self-esteem Support Change & 12 & 1.75 & 1.86 & 8 & 1.63 & 1.41 & 0.08 \\
\hline
\end{tabular}


Table 8 .

Means, Standard Deviations, and Effect Size by Peer Partner BMI Difference.

\begin{tabular}{|c|c|c|c|c|c|c|c|}
\hline \multirow[t]{2}{*}{ BMI Difference } & \multicolumn{3}{|c|}{10 or Less } & \multicolumn{3}{|c|}{ Greater than 10} & \multirow[b]{2}{*}{ Cohen's d } \\
\hline & $\mathrm{n}$ & Mean & SD & $\mathrm{n}$ & Mean & $\mathrm{SD}$ & \\
\hline Age & 12 & 43.00 & 11.77 & 16 & 38.56 & 11.85 & 0.38 \\
\hline Baseline BMI & 12 & 33.61 & 6.69 & 16 & 40.12 & 8.31 & 0.86 \\
\hline$\%$ Weight Change & 12 & -0.34 & 2.71 & 16 & -2.61 & 1.97 & 0.96 \\
\hline Mean Visit/Month & 12 & 8.82 & 2.36 & 15 & 8.88 & 1.29 & 0.03 \\
\hline Mean Contact/Month & 12 & 7.39 & 7.11 & 16 & 5.59 & 5.22 & 0.29 \\
\hline Mean Satisfaction & 12 & 61.25 & 33.19 & 16 & 66.43 & 28.45 & 0.17 \\
\hline ISEL Change & 5 & 13.40 & 4.72 & 13 & 10.46 & 4.74 & 0.62 \\
\hline Appraisal Support Change & 5 & 3.20 & 1.64 & 13 & 2.23 & 1.83 & 0.56 \\
\hline Tangible Asset Support Change & 5 & 5.00 & 1.41 & 13 & 5.08 & 1.71 & 0.05 \\
\hline Belonging Support Change & 5 & 2.20 & 1.30 & 13 & 1.85 & 1.91 & 0.22 \\
\hline Self-esteem Support Change & 5 & 3.00 & 2.24 & 13 & 1.31 & 1.25 & 0.93 \\
\hline
\end{tabular}


Table 9.

Final HLM Model Parameters for BMI Difference in the Peer Sample.

Fixed Effect Coefficient

Standard Error

$p$-value

For Intercept, $\pi_{0}$

$\begin{array}{rccc}\text { Intercept, } \beta_{00} & -0.52 & 1.87 & 0.79 \\ \text { Gender, } \beta_{01} & -7.40 & 3.81 & 0.07 \\ \text { Start BMI }^{*}, \beta_{02} & -0.31 & 0.20 & 0.12 \\ \text { BMI Difference, } \beta_{03} & -6.37 & 2.51 & 0.02 \\ \text { Age }^{*}, \beta_{04} & -0.17 & 0.13 & 0.21\end{array}$

For Linear Slope, $\pi_{1}$

\begin{tabular}{rlll} 
Intercept, $\beta_{10}$ & -0.28 & 0.52 & 0.60 \\
Gender, $\beta_{11}$ & -1.31 & 1.06 & 0.23 \\
Start BMI*, $\beta_{12}$ & -0.08 & 0.05 & 0.17 \\
BMI Difference, $\beta_{13}$ & -1.55 & 0.70 & 0.04 \\
Age* $^{*}, \beta_{14}$ & -0.04 & 0.04 & 0.25 \\
\hline
\end{tabular}

*Indicates variable was centered at the grand mean. 
Table 10.

Means, Standard Deviations, and Effect Size by Peer Partner Age Difference.

\begin{tabular}{|c|c|c|c|c|c|c|c|}
\hline \multirow[t]{2}{*}{ Age Difference } & \multicolumn{3}{|c|}{10 Years or Less } & \multicolumn{3}{|c|}{ Greater than 10 Years } & \multirow[b]{2}{*}{ Cohen's d } \\
\hline & $\mathrm{n}$ & Mean & SD & $\mathrm{n}$ & Mean & SD & \\
\hline Age & 16 & 39.25 & 10.55 & 12 & 42.08 & 13.62 & 0.23 \\
\hline Baseline BMI & 16 & 37.56 & 7.68 & 12 & 37.01 & 9.2 & 0.06 \\
\hline$\%$ Weight Change & 16 & -1.44 & 2.17 & 12 & -1.89 & 3.05 & 0.17 \\
\hline Mean Visit/Month & 15 & 9.14 & 1.76 & 12 & 8.49 & 1.86 & 0.36 \\
\hline Mean Contact/Month & 16 & 8.56 & 6.85 & 12 & 3.43 & 3.05 & 0.97 \\
\hline Mean Satisfaction & 16 & 65.69 & 32.46 & 12 & 62.24 & 27.88 & 0.11 \\
\hline ISEL Change & 10 & 11.30 & 6.17 & 8 & 11.25 & 2.60 & 0.01 \\
\hline Appraisal Support Change & 10 & 2.60 & 1.78 & 8 & 2.38 & 1.92 & 0.12 \\
\hline Tangible Asset Support Change & 10 & 5.00 & 1.94 & 8 & 5.13 & 1.13 & 0.08 \\
\hline Belonging Support Change & 10 & 1.80 & 1.81 & 8 & 2.13 & 1.73 & 0.18 \\
\hline Self-esteem Support Change & 10 & 1.90 & 1.97 & 8 & 1.63 & 1.41 & 0.16 \\
\hline
\end{tabular}


Table 11.

Pearson Correlations with Percent Weight Change at 3-Months.

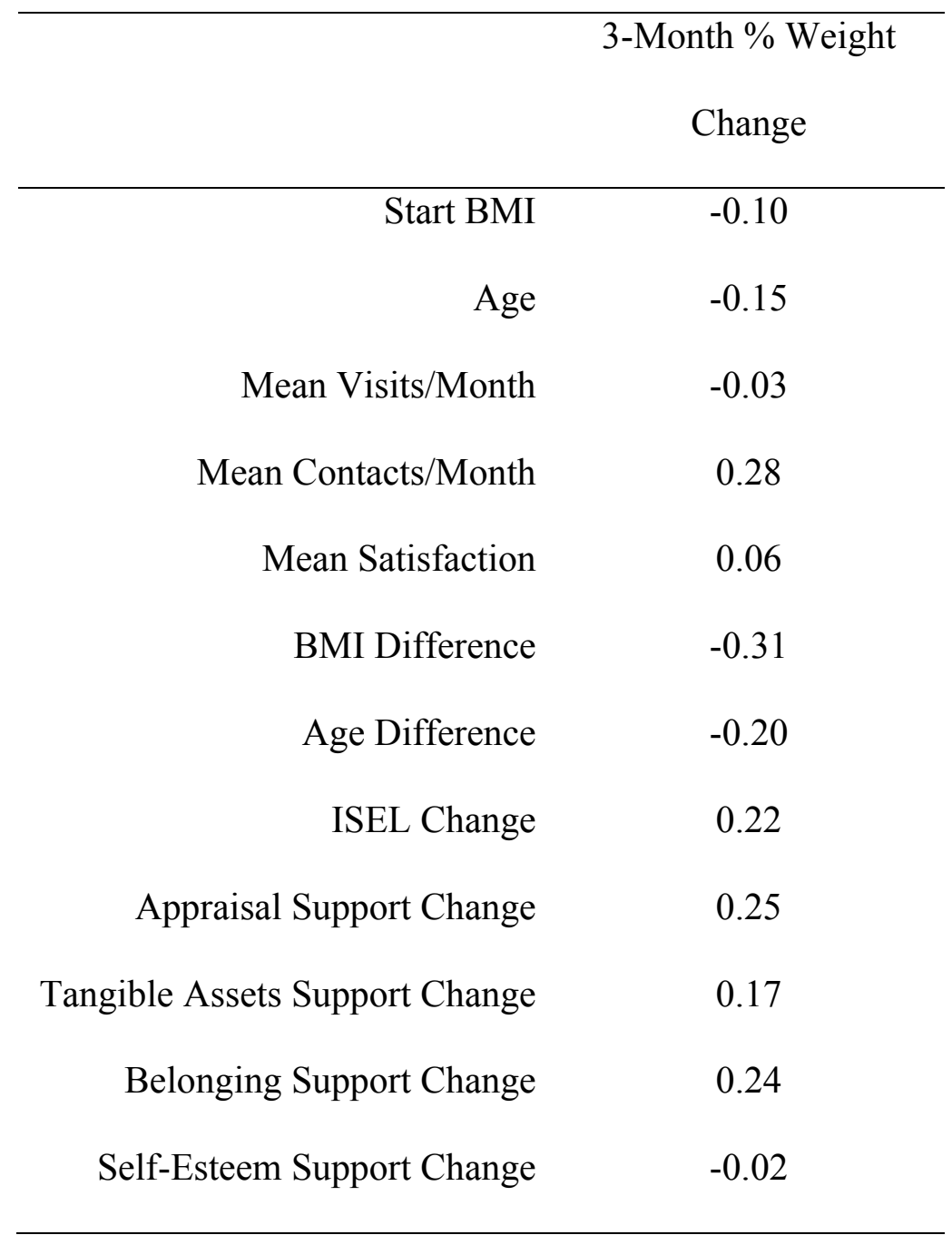

Note. No correlations were significant at the 0.05 level. 
Figure 1.

Distribution of Propensity Scores.

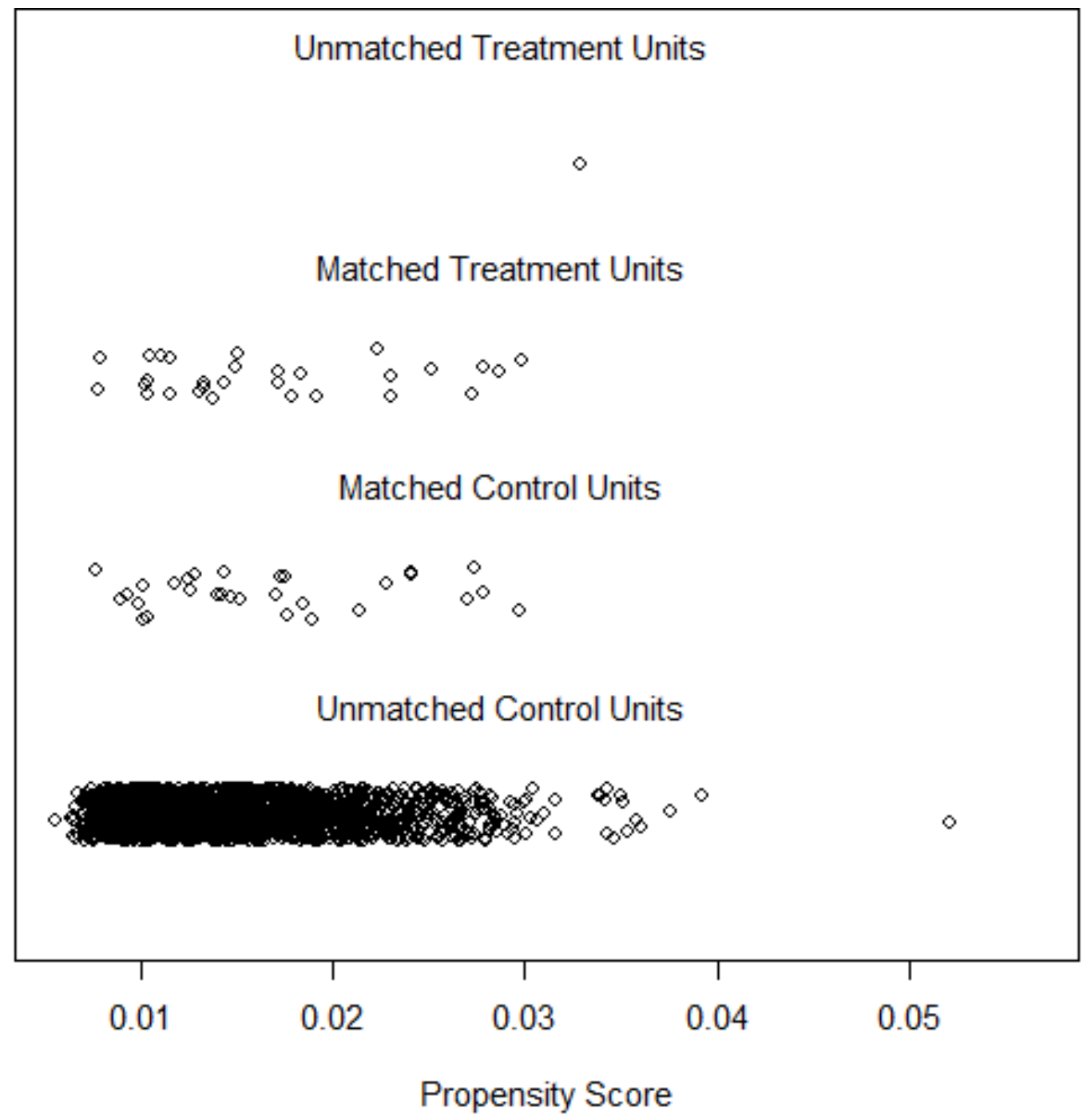


Figure 2.

Graph of Mean Weight Change in Pounds by Month across Peer and Matched Propensity Score Group.

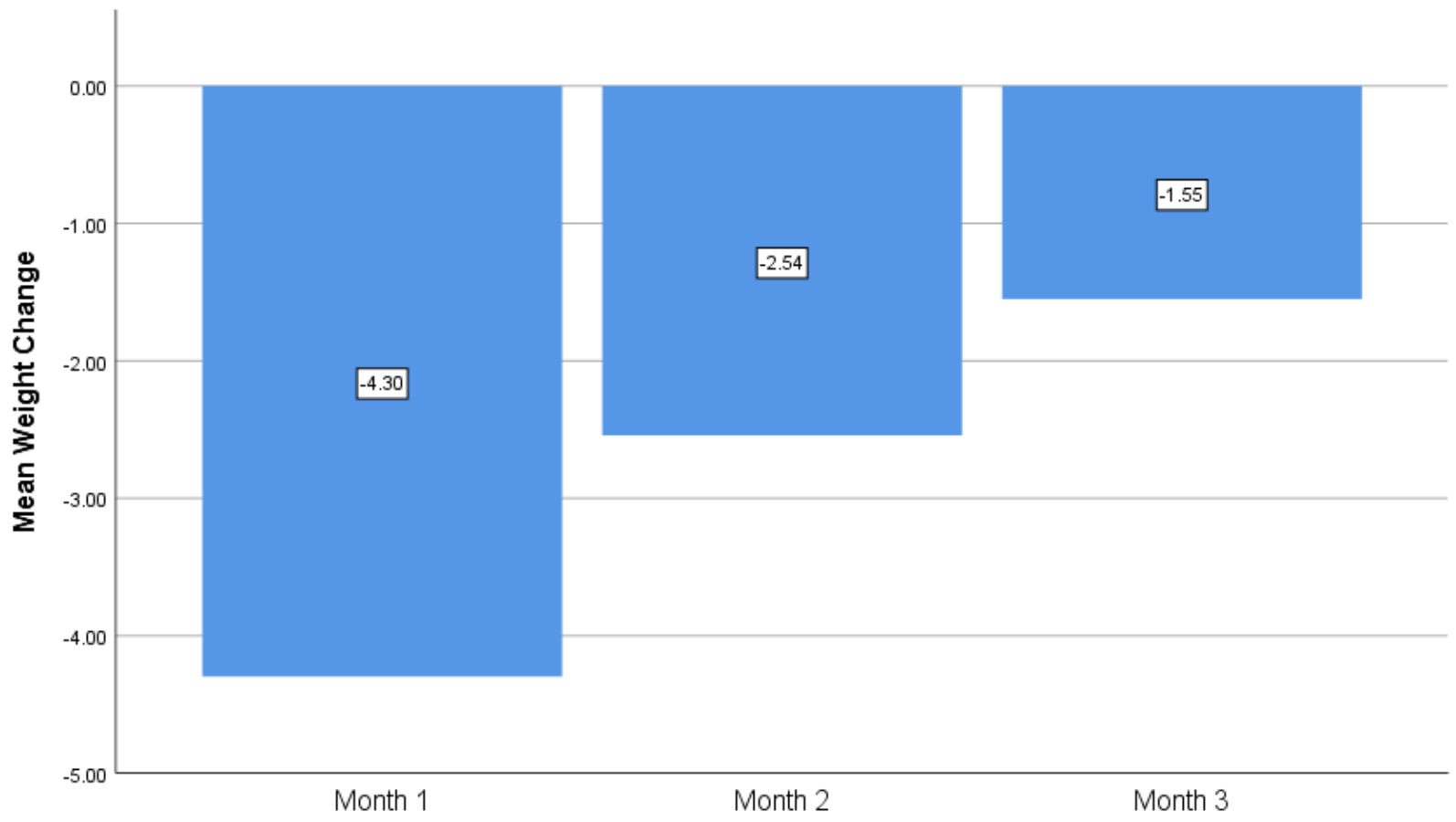


Figure 3.

Histogram of the Distribution of Level-1 Residual Variance for the Full Matched Sample.

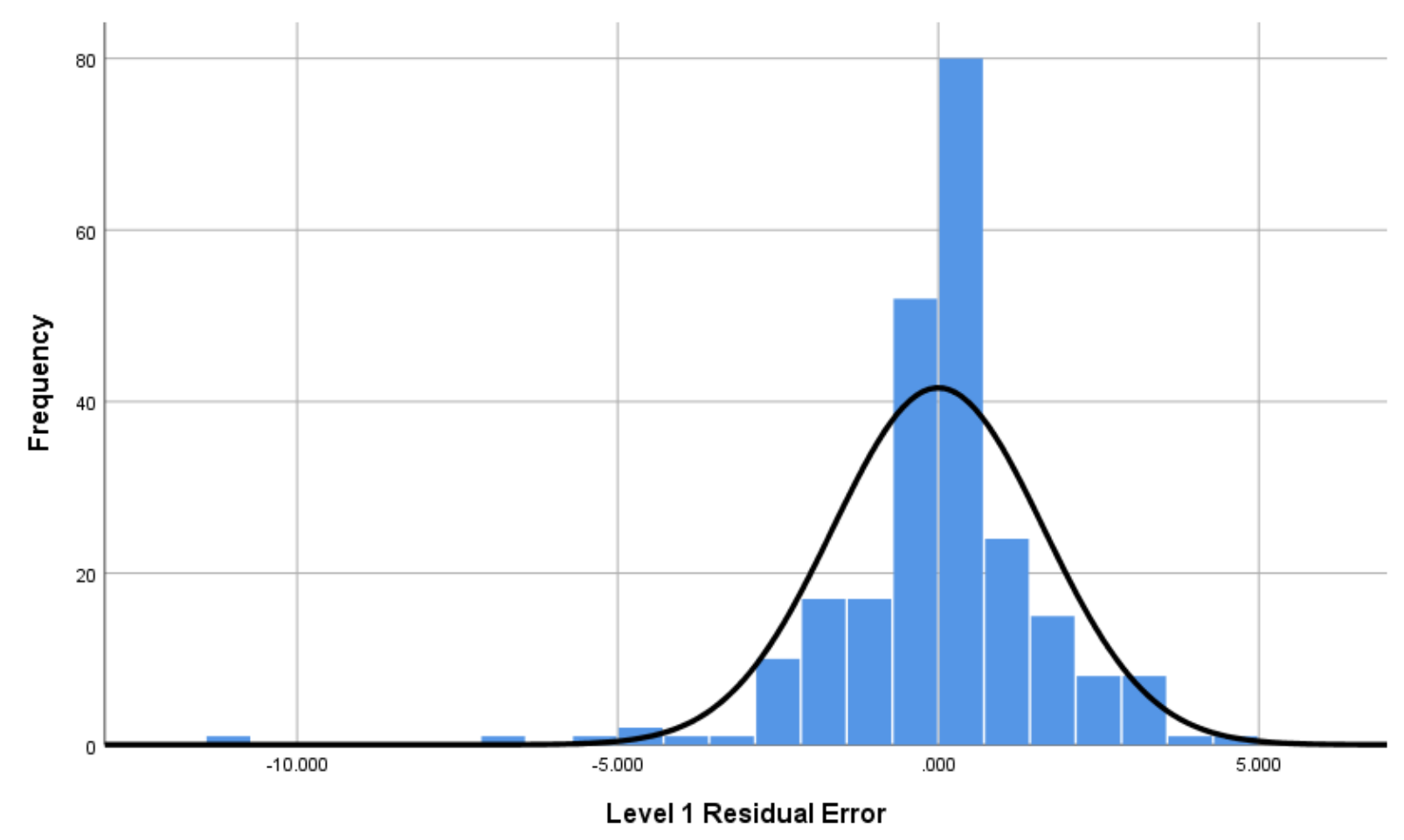


Figure 4.

Histogram of the Distribution of Level-2 Residual Variance around the Intercept for the Full Matched Sample.

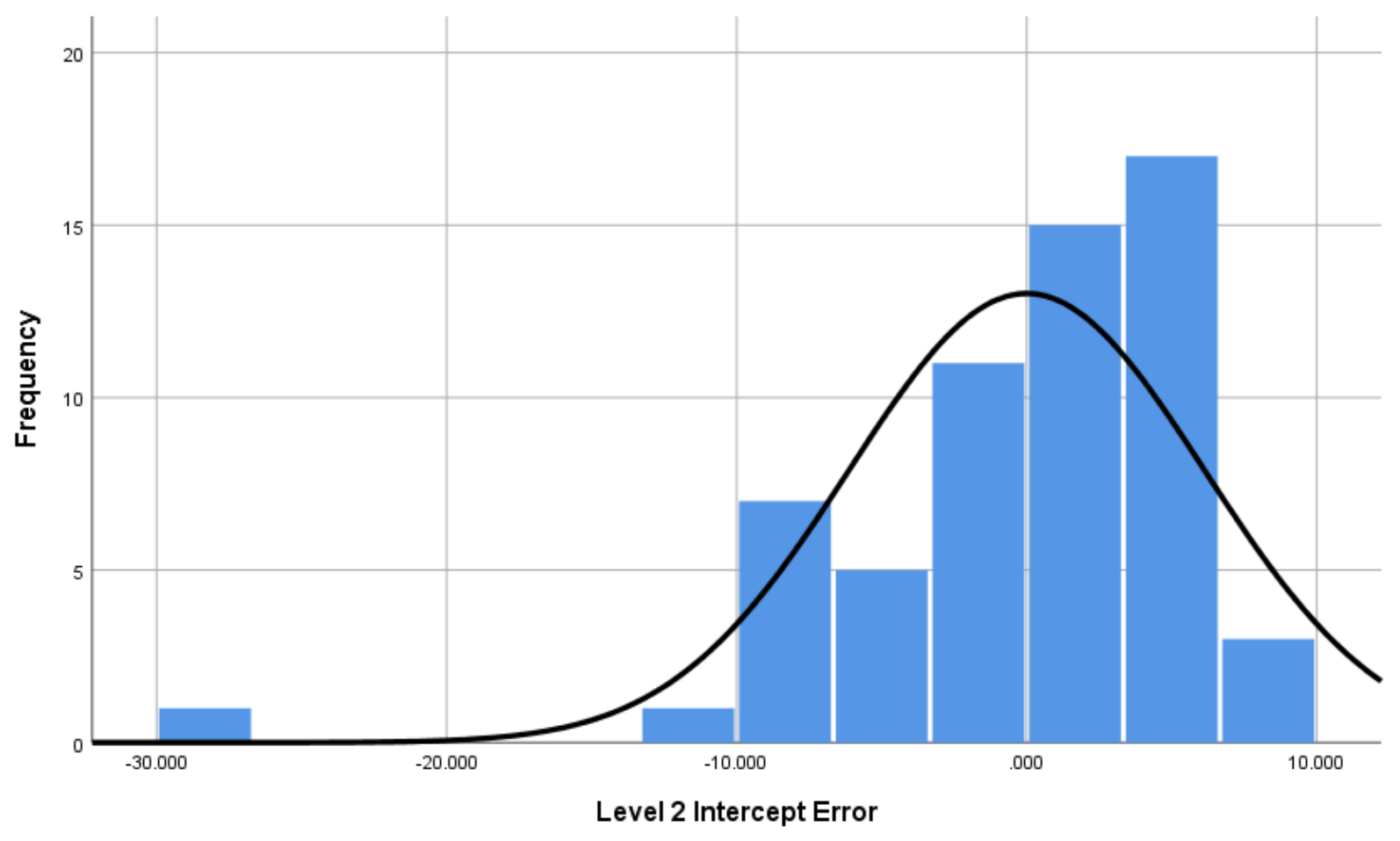


Figure 5.

Histogram of the Distribution of Level-2 Residual Variance around the Slope for the Full Matched Sample.

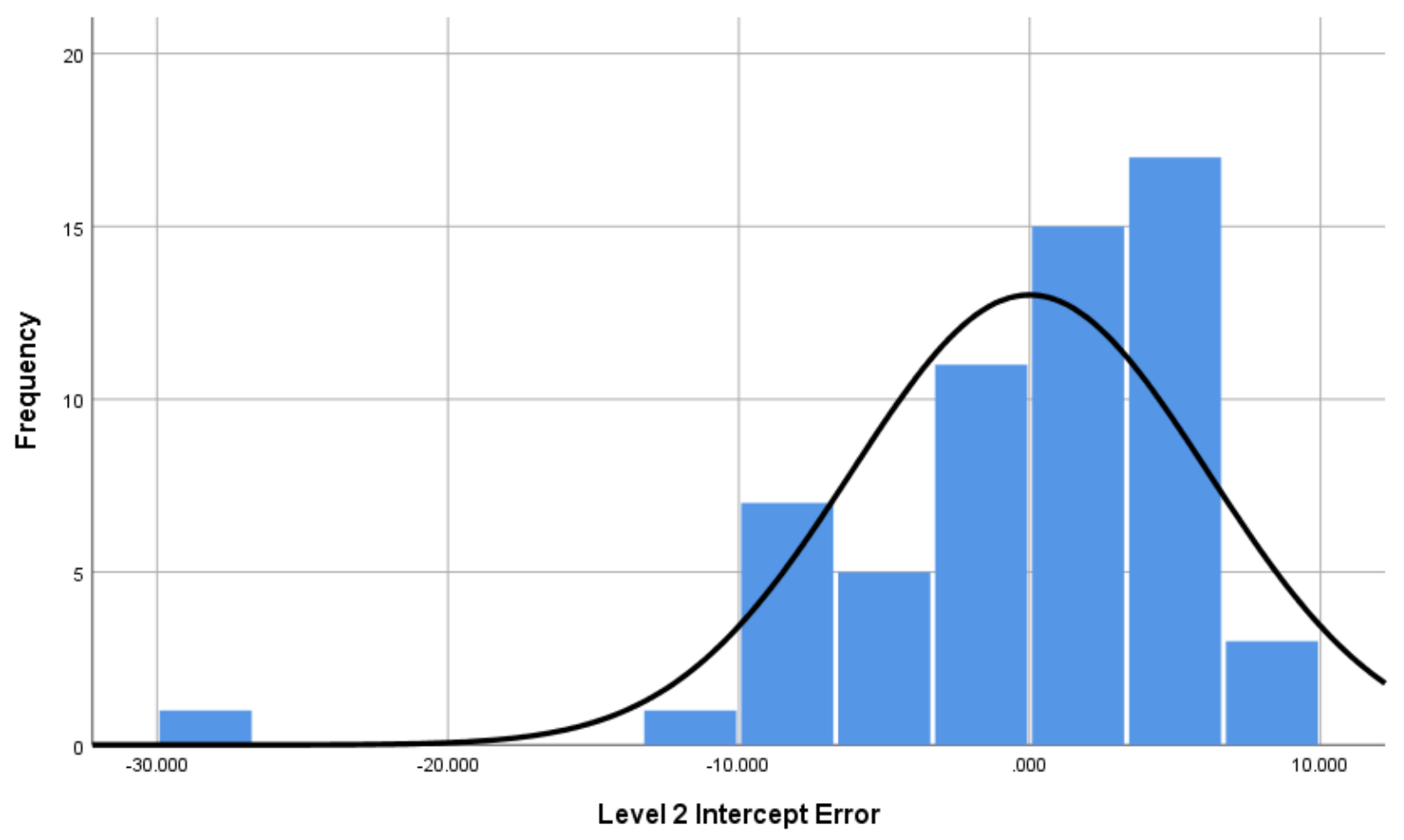


Figure 6.

Histogram of the Distribution of Level-1 Residual Variance for the Peer Sample.

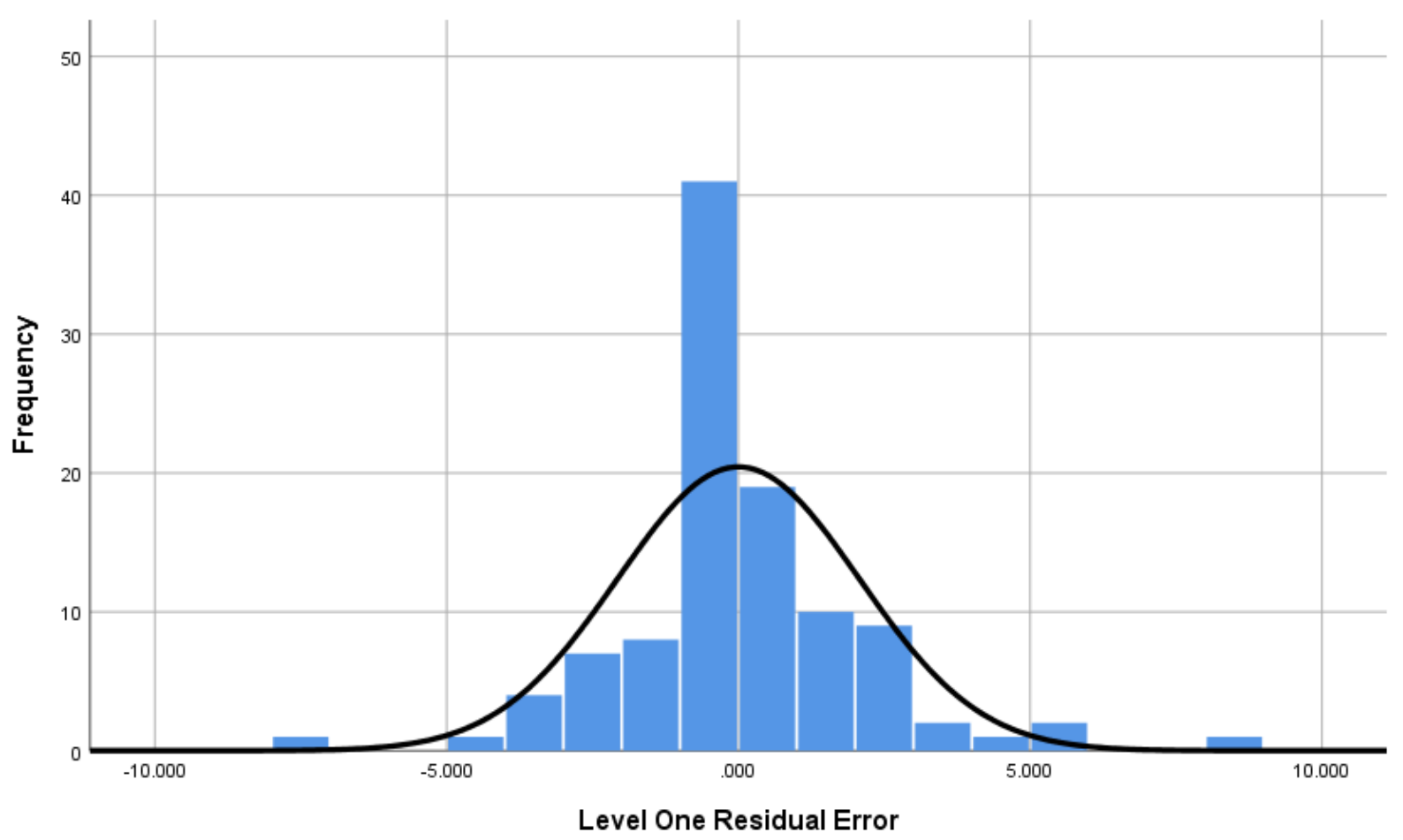


Figure 7.

Histogram of the Distribution of Level-2 Residual Variance around the Intercept for the Peer Sample.

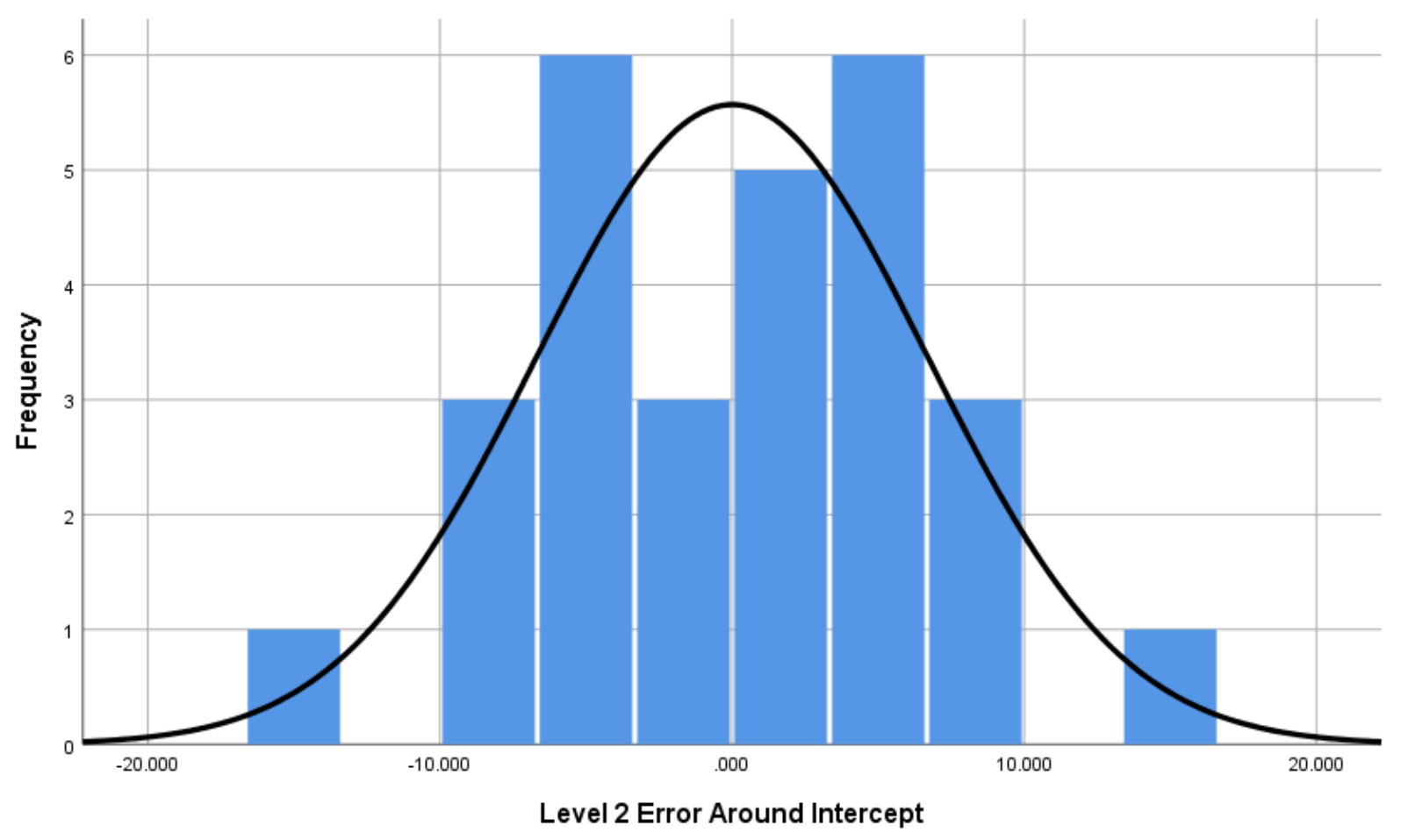


Figure 8.

Histogram of the Distribution of Level-2 Residual Variance around the Slope for the Peer Sample.

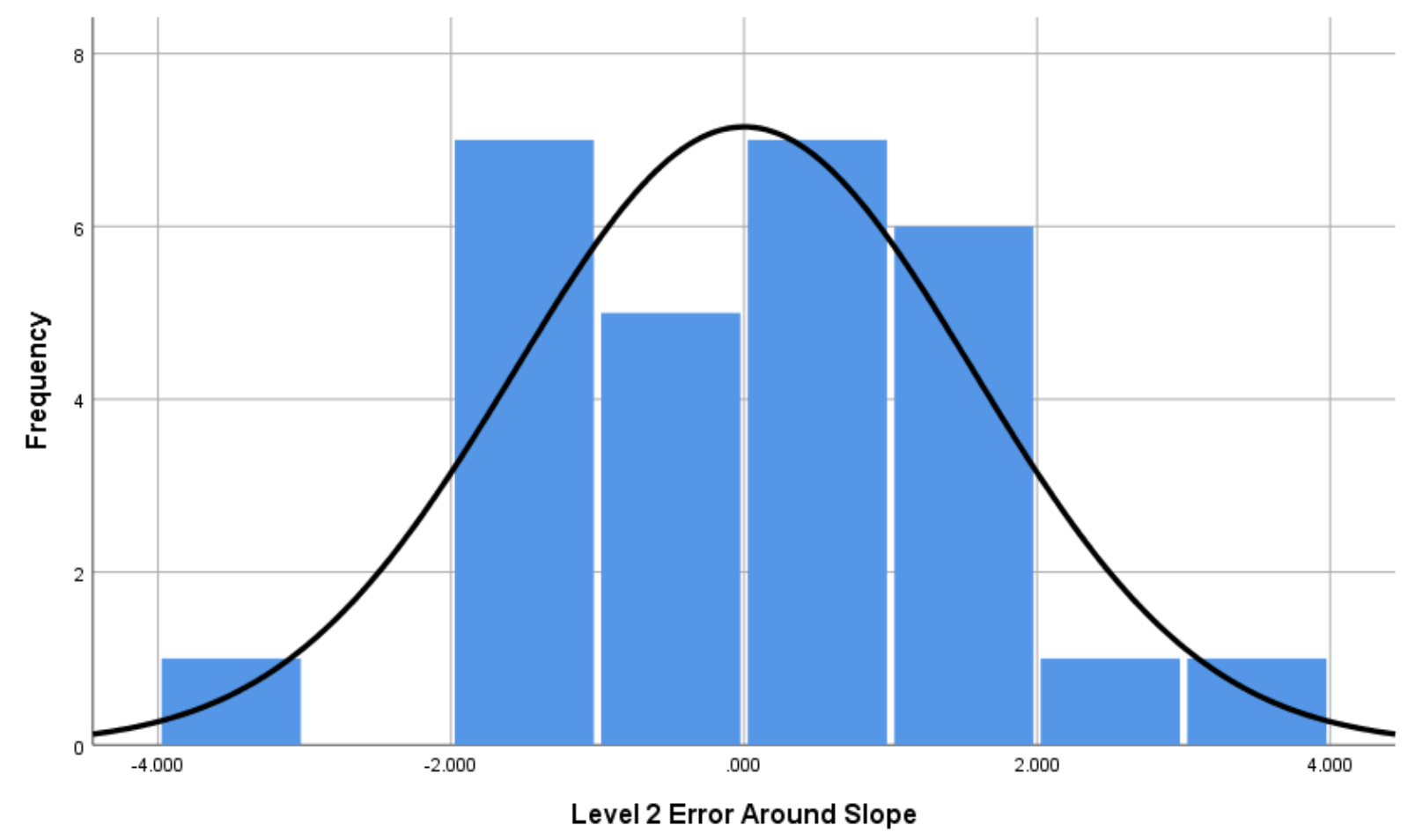




\section{Appendix A - Extended literature review}

Weight-loss has become an industry in the United States, with commercial programs proclaiming to have the solution the newer, thinner you. Evidence has shown that there are many different programs that effectively help individuals lose weight. These programs generally focus on making changes to ones lifestyle, such as adding more exercise, eating healthier foods, limiting unhealthy foods, and managing emotions. The focus of these programs is on helping individuals make changes that result in a healthier lifestyle that they can maintain for the long term, leading to improved health and savings in health care costs. Yet the research on long-term weight maintenance is less promising. While these programs are effective in helping participants lose weight, most weight is regained after the program ends. Understanding why this occurs and developing tools to address this is a major concern for the health care industry and the health of our country.

Social support is one avenue of research that has shown promise in addressing the challenges of maintenance. Evidence suggests that support is closely linked to our health as a lack of support is linked to poorer health, such as weight gain. It is possible that part of the success of lifestyle management programs is the support members receive from the staff and others in the program who share their goals. The research supports this theory, as programs that have focused on increasing support have been shown to result in increased weight-loss for those who participate. The challenge now is to figure out how to provide additional support in efficient and effective ways over the long-term.

One promising area of research has focused on incorporating peers to serve in a support role for individuals working to make lifestyle changes. A peer is someone who is shares a similar background to the individual, and who is able to provide support within a variety of areas. Many 
different types of peers have been used, from community health workers who share a culture background, to peer leaders who have already lost weight, to peer partners who provide support while they themselves are working to improve their health. Most of the research in this area has focused on peer support within diabetes management, which has shown promise for the use of peers instead of, or in addition to professional support. Peer support has been shown to be a promising option for increasing the available support for individuals working to improve their health by making changes to their lifestyle. However, there are few projects that have focused on the use of peer support within weight-loss settings, which presents an area that requires more research. Further, the training of peers has varied greatly across studies, thus it is unclear how much and what kind of training peers actually need to successfully support each other. Finally, research is needed to understand how to match peers in order to provide the most effective support. For example, do women do best when matched with another woman? Does weight, health status, and age matter when matching two individuals? Answering these questions will improve the effectiveness of peer support programs.

This review will first aim to give an overview of the effectiveness of lifestyle interventions in addressing weight-loss and weight maintenance. It will then review evidence of the role social support plays for an individual's health, specifically focusing on weight-loss. Finally, peer support will be defined and evidence from multiple studies will be reviewed in detail. The research focused on peer support within diabetes management will be explored in detail, as this represents the majority of peer support literature. Further, while diabetes management differs from weight-loss, there are commonalities in the behavioral changes needed to both manage diabetes and lose weight, such as making changes to diet and physical activity. Thus, this diabetes research can inform future research focused on peer support within weight- 
loss. Despite the majority of research being focused on diabetes management, a handful of studies have focused on peer support within weight-loss. This review will conclude by focusing on these studies and the future directions for researching peer support within weight-loss.

\section{Lifestyle Interventions for Weight-loss}

Over the past 30 years chronic diseases, like obesity and diabetes, have grown in prevalence in the United States as currently over 75-percent of the population in the United States are classified as overweight or obese (Fryar, Carroll, \& Ogden, 2016). This has placed a large burden on the health care system to provide the resources necessary to treat these diseases and the multiple comorbidities that accompany them. Lifestyle interventions targeting weightloss been shown to effectively lower weight of those who participate. The Diabetes Prevention Program (Knowler et al., 2002) is one widely cited intervention that directly compared the effectiveness of a lifestyle intervention, which included physical activity and dietary change, with a leading drug for diabetes which has also been shown to result in weight-loss. Content for the lifestyle intervention was presented in a group format over 24 weeks and 16-sessions, covering diet, physical activity, and psychological content focused on initiating and sustaining lifestyle change. In addition, participants met individually with case managers throughout the program for support in making and sustaining lifestyle change in their lives. A sample of 3,234 of participants at risk of developing diabetes, comprised of 32-percent males and 46-percent nonwhite ethnicities, were randomly assigned to either the lifestyle intervention $(n=1,079)$, Metformin $(n=1,073)$, or placebo control group $(n=1,083)$. Those in the lifestyle intervention made significant changes in their daily caloric intake and physical activity levels over the 24week program, which resulted in $50 \%$ of the sample reaching at least seven-percent weight-loss 
at 24-weeks, and average weight-losses of over 5 kilograms in the lifestyle group, significantly more than the metformin group $(-2 \mathrm{~kg})$ and control group $(-0.1 \mathrm{~kg})$.

The DPP is only one example of the many programs that have been implemented. A unique weight management program has been run through the Public Employees Insurance Agency (PEIA) of West Virginia for their members who are classified as overweight. The program provides discounted gym membership, access to a registered dietician, personal training sessions, and phone coaching from a health-behavior coach (Zizzi et al., 2014). Evaluation of the program found that 75-percent of participants completed the first three months, achieving weight-loss of 13 pounds on average, and maintenance of this weight-loss was good as over 50\% maintained weight-loss or continued to lose over the following nine-months in the program (Abildso, Zizzi, \& Reger-Nash, 2010). Those who completed the full 12-month program (46\% of 762 participants) lost an average of almost seven-percent of their baseline weight.

This highlights two of many interventions that have reported on the outcomes of lifestyle interventions impact on participant's weight and health outcomes. The growing need for weightloss interventions has led to the development of commercial weight-loss programs. A 2005 review of multiple commercial weight-loss programs found that Weight Watchers (WW) was the only program to have their efficacy tested in a large, multisite, randomized controlled trial (Tsai, 2005). They reported that those who joined WW lost an average of five-percent of baseline weight after one year in the program and were able to maintain a three-percent loss after two years. Further, those who attended the most group sessions over the two-year study period maintained the largest weight-losses, highlighting the importance of attending the group sessions to each member's success in weight-loss and maintenance. It is possible that the group sessions provided a source of support in staying focused and engaged with their weight-loss goals. 
The quantity of research in this area has led to multiple systematic reviews and metaanalyses. A 2013 review of 67 papers found that the behavioral strategies implemented in both the DPP and PEIA interventions, such as guidance on making dietary changes, increasing physical activity, and support with behavioral strategies to make these changes happen are common weight-loss strategies across interventions (Ramage et al., 2013). While a 2009 review found that studies who incorporated more self-regulation practices (e.g. goal setting, selfmonitoring, emotion control, coping) resulted in greater weight-loss outcomes (Huisman et al., 2009). Further, goal reformulation, or being able to adjust an unrealistic goal, was found to moderate participants weight-loss, with interventions that focused on goal reformulation resulting in improve participant outcomes.

Similar results have been found in subsequent meta-analyses. A 2007 review looked for RCTs of 16-weeks or more, with overweight participants who were provided dietary counseling to achieve weight-loss (Dansinger, Tatsioni, Wong, Chung, \& Balk, 2007). They identified 46 trials, with 6,386 participants receiving dietary counseling and 5,467 receiving usual care, with BMI ranging from 25 to 40, and age ranging from 27 to 68. Dietary advice along with exercise was provided in 42 of the 46 trials. The delivery format varied with 18 trials using a group format, 13 individual, and 11 a combination of both (three used the internet and two did not specify). Across all studies, participants who received dietary counseling, across all delivery formats, lost an average of six-percent of their baseline weight at 12-months, equivalent to a drop of almost two BMI units. In addition, they reported that participants lost an average of $0.08 \mathrm{BMI}$ units per month during the active portion of the interventions and regained between 0.02 to 0.03 BMI units per month during the subsequent maintenance phases, suggesting that on average participants would return to baseline after about five and a half years. A 2007 review of 80 RCTs 
of weight-loss programs with at least a one year follow-up found that programs focused on diet alone, diet and exercise, or meal replacements resulted in mean weight-loss of five to ninepercent of baseline weight at six-months, five to eight-percent at 12-months, and approximately three to four-percent loss at months 24, 36, and 48 (Franz et al., 2007). Showing that clinically significant weight-loss can remain for up to four years. Finally, a systematic review of reviews of interventions focused on diet and/or physical activity found weight-loss of three to five kilograms at 12-months and two to three kilograms at 36-months across 30 reviews and 3,856 articles (Greaves et al., 2011).

While these results are promising it is a concern that participants tend to lose the results they achieved in the program after the program ends (Abildso et al., 2014; Peirson et al., 2015). A meta-analysis of 45 weight maintenance studies found that weight maintenance could be improved for up to two years post weight-loss intervention through additional support provided by the weight maintenance interventions (Dombrowski, Knittle, Avenell, Araújo-Soares, \& Sniehotta, 2014). One possible explanation is that participants struggle to maintain their weightloss when the support they receive through the lifestyle program ended. Results from two metaanalyses provide support for this explanation as they found that interventions that include a support component result in better outcomes. First, an analysis of 34 interventions with an average length of 44-weeks, across 5,469 participants with type-2 diabetes (T2DM) found that interventions which included a participants partner or relative resulted in significantly greater weight-loss (Huisman et al., 2009). Second, in the a review of reviews cited earlier it was found that adding social support to interventions resulted in three kilograms of additional weight-loss at 12-months versus the same intervention without support (Greaves et al., 2011). These results point to several key factors. First, lifestyle interventions targeting weight-loss help individuals 
lose weight. Second, maintaining this loss once a program ends is difficult. And finally, they show that incorporating additional support into these programs results in improved outcomes.

\section{Social Support and Health}

Support has been studied in multiple health care settings and in general has been linked to improved health. For example, one prospective of a cohort of 846 married individuals who were tracked over a five-year period to measure the impact of both giving and receiving support on mortality (Brown, Nesse, Vinokur, \& Smith, 2003). After controlling for age, gender, personality traits, socioeconomic status only giving support, not receiving, resulted in a $30-40 \%$ reduction in risk of mortality. This reduction may be a result of the important role support plays in adherence to treatment as a meta-analysis of 122 studies found odds of adherence was three times higher for those in cohesive families and almost four times higher for those who receive support than for those who do not (DiMatteo, 2004). Support plays an important role in behavior change that lead to improved health. The remaining studies in this section will focus on the role support plays in weight management.

Members of the Framingham Heart Study, a large prospective cohort study spanning 32years and tracking over 12-thousand participants, were studied to examine the spread of obesity (Christakis \& Fowler, 2007). Using data from multiple points across the 32-years they were able model individuals' social networks over time, and track how these networks changed. The results showed that social distance between connections in ones social network, but not geographic distance between friends and family, significantly increased risk of obesity. The risk of obesity increased by $45 \%$ if someone within one-degree of separation became obese, risk increased by $20 \%$ for a second degree connection becoming obese, and $10 \%$ for a third degree connection, beyond that no significant increase was noted. Further, when looking at geographic distance of 
connections no significant increases in risk were found. Among brothers, if one brother became obese there was a $44 \%$ increase in the risk that the other would become obese, for sisters the risk increased by $67 \%$. Among married couples there was $37 \%$ increased risk of obesity if one spouse became obese, and this risk did not significantly differ if the husband or wife became obese. Most striking, if a mutual friend became obese there was an increase of $171 \%$ that the other would become obese. These results point to the influence our social networks play in in our health-behaviors. We cannot simple blame a toxic environment since geographic location did not influence obesity risk, instead we must look for ways to change the social networks to improve health.

Two studies have explored the role of support plays for members in online weight-loss communities. The first used a combination of open-ended survey questions and interviews to better understand how members of Sparkepeople supported each other (Hwang et al., 2010). A group of 193, primarily white and female participants completed the survey. They reported that they frequently read members messages and blog posts for support with their weight-loss, and $60 \%$ said that members of Sparkpeople were more helpful than other people in their lives when it came to weight-loss support. Members gained support in the form of information, encouragement, motivation, shared experience, and they reported enjoying being able to provide support to others. The shared experience appeared particularly helpful as they reported it was different from interactions with others in their lives as members, who had similar goals, were able to empathize and support each other in new ways.

A second study conducted semi-structured interviews with 12 women and six men who used Livestrong.com to lose weight (Wang et al., 2015). Eight of these members were currently focused on losing weight, and the remaining ten were focused on weight maintenance. All 
participants indicated that receiving social support from other members was important to their weight-loss success, but that support significantly decreased as participants approached their maintenance weight. Further, participants valued receiving support from a peer who had similar health-related experiences, whom they could relate to, empathize with, and were knowledgeable of underlying similarities in their situation. Finally, maintainers were helped by supporting others who were still trying to lose weight, as this helped them remember the struggles, discomfort, and stigma they had experienced which motivated them to stay vigilant and prevent weight regain. These studies show that support is perceived to play a large role in the weight-loss journey of participants focused on losing weight.

Two relevant studies have manipulated the support provided to participants in weightloss programs. The first analyzed weight-loss outcomes across four conditions based off of two recruitment strategies and two intervention strategies (Wing \& Jeffery, 1999). Participants either enrolled in the 10-month program alone or with a group of friends, family, or coworkers; they were then randomized to receive a standard behavioral weight-loss intervention alone or the standard intervention plus a support component. A total of 166 participants $(\mathrm{BMI} M=31.2, S D=$ 3.7, age $M=42.5$ years, $S D=8.5)$ evenly split between males $(n=82)$ and females $(n=84)$ were randomized across conditions. The standard intervention was delivered in 16-sessions over four months focusing on diet, exercise, and behavior modification strategies. Follow-up was conducted at months seven and ten. Those in the support condition operated as a team for the group sessions as they sat together, created a team name, shared contact information and were told to contact each other between sessions to provided support in reaching their goals. In addition, each week after the lecture was given on the topic teams were given an assignment to work on together, which they then reported back to the bigger group at the end of the session. 
Finally, those in the support condition were involved in an intergroup competition at the completion of the four-month intervention period, as teams with the greatest proportion of members retaining their weight-loss in full would win a monetary prize. The social support groups were comprised of those who enrolled with their team and those who enrolled alone but were assigned three other members to create their team. Likewise, for those receiving only the standard treatment there was a group recruited alone and a group recruited with friends but who received to further direction in working as a team to support each other. Several results point to the importance of support. First, regardless of intervention condition, those who enrolled with a group versus those who enrolled alone lost over two kilograms more during the first 16-weeks of the study. Second, these results remained significant at 10-months as those recruited with a group had lost 33-percent more weight than those recruited alone. Third, significantly more participants who were recruited with a group and received the support intervention completed the 10-month assessment. Fourth, those who enrolled alone and received standard treatment had significantly worse outcomes than all other groups, highlighting the role social support plays in weight-loss and weight maintenance. Finally, they found that higher positive family support for healthy eating and exercise was related to poorer weight-loss from baseline to month ten, while for the groups receiving support higher perceived support from others in their group was related to greater weight-losses at month ten. Indicating that the support received from others in the program was more influential on weight outcomes than support from family.

The second study explored the role of support in weight-loss by comparing a group who received a standard cognitive behavioral weight-loss program and those who received the same program plus support people trained in motivational interviewing (Rieger et al., 2017).

Participants, between 18 and 65 years of age with a BMI over 30, were randomly assigned to the 
standard $(n=103)$ or the support intervention $(n=98)$. Those in the support condition recruited someone from their social network (partners, siblings, adult children, parents, friends, and colleagues) to attend a program designed for the support people. The support people program consisted of ten 90-minute group sessions, which trained them to use motivational interviewing to help bring out their partners own ability to manage their weight. Both groups lost a significant amount of weight over the 12-month treatment period, but no differences were found between groups in weight-loss or BMI, however higher perceived support from a partner at baseline was associated with a greater decrease in weight and BMI from baseline to post-treatment. Further, there was a significant decrease in ratings of relationship support from baseline to post-treatment, indicating that the support participants received at baseline was more important to participant outcomes than the training the support members received during the intervention. These results point to the important role support plays in weight-loss, yet training of individuals to provide support is difficult. However, the broad range of supporters may have limited the ability of supporters to empathize with their supportees weight journey, which may have impacted their ability to successfully provide support. Finally, the significant association of baseline support with final weight improvement could be explained by supporters having inherent skills that may not require additional or extensive training in order to provide effective support. The important role of support within weight management creates a need for new methods to increase effective support to the ever-growing population of weight management participants. One area that has shown promise is incorporating peers to provide support, which will be reviewed later.

Theories linking social support to improved health. Dennis (2003) explores several models to help understand how support benefits patients: the direct effect model, the buffering effect model, and the mediating effect model. The direct effect model posits that support can 
influence health outcomes by decreasing isolation and feelings of loneliness, improving health practices by deterring maladaptive behavior, promotion positive psychological moods (e.g. motivation), providing information on medical or community resources and the benefits of engaging in behaviors that will positively impact health and well being, and finally preventing the risk of progression of disease. The results from several studies lend support to the direct effect model. Analysis of phone calls by peer supporters show that the most common topics covered were diet, exercise, self-monitoring, self-care, and medication use, suggesting that the content of peer support focus directly on health-behaviors that improve health outcomes (Chan et al., 2014). Another study revealed that coaches helped motivate, encourage, and support patients in engaging in healthier lifestyles, by, for example providing useful tips about eating (Dutton et al., 2015). Further semi-structured interviews with participants receiving peer support revealed that patients felt phone calls gave them a opportunity to reflect on their diabetes management and establish goals, which increased their confidence in being able to manage their diabetes (Dale, Caramlau, Sturt, Friede, \& Walker, 2009). Finally findings in a study from Moskowitz et al. (2013) found that peer support had the greatest effect for participants with lower levels of selfmanagement of diabetes, as peer supporters may help by modeling and teaching new selfmanagement strategies.

In contrast to the direct effect model, the buffering effect model states that support acts as a buffer to life stress. This buffer can either protect an individual from the potentially harmful effects of stressful events or support an individual to respond in positive ways to stressful events. Thus, this model supports a dual coping process of problem solving when possible and emotional support and regulation when source of stress does not have a solution. Peers can act as a buffer by redefining and reducing potential harm of a stressor, increasing coping resources, discussing 
coping strategies, highlighting role models of adaptive behavior, inhibiting maladaptive responses, and finally counteracting the tendency to blame oneself for causing the stress or adversity. For example, results from one RCT found that participants with T2DM who were partnered with a peer showed higher rates of insulin therapy, suggesting that patients concerns about insulin may be best addressed through support from another person who is also coping with insulin management (Heisler et al., 2010). This finding points to the importance of role the similarity in backgrounds and health conditions can play in helping peers feel supported and understood while dealing with making lifestyle changes to improve their health. Participants have reported the common understanding and support they receive from a peer are some of the most important aspects of peer support programs (Long, Jahnle, Richardson, Loewenstein, \& Volpp, 2012), lending support to the buffering hypothesis claim that the support from peers is the driving force behind the health improvements. Interviews from another study found that participants reported the connection with their peer leader was helpful in improving their health, with leaders who provided emotional support were reported to be especially beneficial (Albarran, Heilemann, \& Koniak-Griffin, 2014). Further, participants strongly connected improved mental well-being with their ability to lose weight and be healthier, supporting the important role peers can play as buffers to life stress.

In contrast to both the direct and buffering effect models, the mediating effect model predicts that peer support indirectly intervenes to impact health by effecting an individuals emotions, cognitions, and behaviors (Dennis, 2003). Peer relationships can indirectly influence health-behavior by assisting in appreciating and reinforcing individual accomplishments, provide learning and new experiences through role modeling, providing opportunities for social comparison that promote positive self-evaluations that build motivation, teaching coping 
strategies, building emotional awareness and learning how positively interpret emotional reactions, and finally supporting restructuring cognitions to support positive health-behaviors.

Several other theories have been developed that help explain the influence of social support on health. First, a theory conceptualizes social support impact on health as a complex process involving multiples pathways (Heaney \& Israel, 2008). Finally, a recent theory explores social support influence on health by focusing on support with adversity and support for growth (Feeney \& Collins, 2015). These will be reviewed in more detail next.

The first theory defines four types of support behavior: emotional, instrumental, appraisal, and informational (Heaney \& Israel, 2008). Emotional support involves providing empathy, love, trust, and caring. Instrumental support provides tangible aid and services that directly help the person (e.g. money, transportation). Appraisal support provides constructive feedback and affirmation aimed at supporting self-evaluation. Finally, informational support provides advice, suggestions, and information that the person can use in solving their problems. These types of support are theorized to impact health through five pathways. First, a direct effect pathway posits that support, by meeting basic humans needs for connection, enhances well-being and health. Second, support buffers impact of negative life circumstances by increasing individuals coping resources. Third, support can influence the frequency and duration of exposure to negative life circumstances. So having support can help limit the amount of negative life circumstances one may face, thus improving health by decreasing the amount of stress one has to cope with. Fourth, social support can increase the quantity of coping resources within a broader community, improving a community's ability to respond to life challenges. And fifth, social support has a direct effect on an individual's ability recover from disease or illness. Thus, this model builds upon prior research by combing buffering and direct effect of social support on 
health and focusing on these effects at an individual and community level. Further, they theorize that social support does not follow a typical linear relationship, with increased health support resulting in ever increasing health. Rather, there is a cut-off point where a lack of support results in poor health outcomes and very high support has a minimal effect on health improvement after a threshold level of support has been met. A broad range of individuals can provide support, from professionals to family to peers, and what is most important is how support is perceived rather than objective measures of the quantity of support. Finally, the type of support that will be most effective in improving health depends on an individual's age and developmental stage.

The final theory proposes that social support effects health by increasing the degree to which individuals thrive in their lives (Feeney \& Collins, 2015). They define thriving as having happiness, satisfaction, life purpose, and social, physical, and psychological well-being. A unique contribution of this theory is the inclusion of how social support influences thriving in the face of both adversity and times of non-adversity, or opportunities for growth. The theory posits that support can influence health not just when facing stress or adversity (e.g. buffering), but also at times when individuals need support to embrace opportunities for growth or change in their lives. The authors define two types of support in relation to these two situations: Source of Strength (SOS) for adversity and Relational Catalyst (RC) for growth opportunities.

SOS support operates by both buffering the effects of adversity and through supporting an individual to grow through adversity. The following four components are involved in providing effective SOS support. First, providing a safe haven involves supporting individuals to feel safe and protected to share any negative emotion or vulnerability, providing relief from burdens, providing emotional or physical comfort, and expressing empathy, understanding, acceptance, and reassurance. Second, individuals provide fortification by helping individuals 
develop new talents, strengths, or abilities, or by supporting the ongoing development of existing talents, strengths, or abilities. Third, individuals assist with reconstruction by motivating and assisting an individual to get back up and stay focused on goals in the face of adversity. Finally, effective SOS support involves assisting individuals in reframing or redefining adversity as a positive opportunity for change.

RC support promotes thriving by helping individuals embrace opportunities for growth and development in the absence of adversity. Again there are four components to providing effective RC support. First, individuals nurture a desire to create and take on opportunities for growth by expressing enthusiasm, validating goals and aspirations, and challenging individuals to extend beyond their comfort zone. Second, individuals provide assistance in perceiving life opportunities by helping recognize situations for growth and helping perceive these situations as opportunities for growth. Third, individuals help facilitate engagement in opportunities for growth by promoting development of plans and strategies for growth, identifying skills and resources already in place, and providing instrumental or informational assistance. Finally, individuals provide effective RC support by providing a safe space for supportees to launch into their areas of growth. This goal is accomplished by providing a secure base from which a supportee feels safe to explore and grapple with the challenges of growth, such as responding with sensitively and caring to setbacks or challenges that arise and supporting behavior that is consistent with the ideal self the supportee is striving for.

Overall, these theories offer a framework for understanding different pathways by which support can influences health outcomes. It is likely that in practice having a supporting other has both direct and mediating effects on health outcomes, as the support may directly improve health through changes or connection to resources, while also improving health indirectly by 
influencing behavior change. Given the complex nature of social support the model presented by Feeney and Collins (2015) provides practical advice for providing support to individuals in weight-loss settings. Of particular relevance is the distinction between support in the face of setback or challenges and support to embrace positive change. This distinction can help inform what type of support is most effective depending on the situation facing the person. For example, someone who is coping with a recent negative health diagnosis related to their weight will need different support than someone who is otherwise healthy but is struggling to commit to lifestyle change to lose weight (i.e. eating healthier and increasing activity).

Social support measures. Peers provide a great opportunity for providing on-going support throughout the challenging process of initiating and sustaining lifestyle change. There are several assessments will be useful in understanding the impact of peer support. First, since peers would be providing additional support to their partner it is important to assess each participants baseline level of support, as this will likely impact the effectiveness of having a peer partner. For example, a peer who already has a high level of support in their life may not experience as strong of an effect from a peer as someone with low levels of peer support. Thus incorporating a social support assessment, like the Multidimensional Scale of Perceived Social Support (MSPSS, Zimet, Dahlem, Zimet, \& Farley, 1988), the Social Support Questionnaire (SSQ, Sarason, Levine, Basham, \& Sarason, 1983), or Interpersonal Support Evaluation List (ISEL, Cohen, Mermelstein, Kamarck, \& Hoberman, 1985) are necessary to better understand the effectiveness of peer support.

The MSPSS consists of 12 items total items with four seven-point likert scales assessing perceived social support across the three subscales of family, friends, and significant other. The MSPSS was psychometrically tested on sample of 275 undergraduate students in a introduction 
to psychology class at Duke University who completed the assessment for class credit (Zimet et al., 1988). Internal reliability was 0.88 for the entire scale and 0.85 for test-retest reliability. Validity shown through correlations between increased support and decreased anxiety and depression levels.

A second tool that has been used to assess social support is The Social Support Questionnaire (SSQ, Sarason et al., 1983). The goal of the scale is to capture the quantity and satisfaction of social support by having individuals list the number of people they can count on for support in a given circumstance and ranking their level of satisfaction with this support. For example participants are asked: "whom can you really count on to listen to you when you need to talk?" or "with whom can you totally be yourself?" (Sarason et al., 1983, p. 129). There are 27 total circumstances make up the items of the full scale, with each item having two parts. First, it asks participants to list the people who they can turn to or rely on given the set of circumstances each item lays out, and second, to rate their level of satisfaction with the level of support they receive from one (low) to six (high). The scale was psychometrically assessed on a sample of 602 undergraduate students at the University of Washington (Sarason et al., 1983). The Cronbach alpha's for the Number and Satisfaction scales were very high, 0.97 and 0.94 respectively. High re-test reliability for both the Number $(r=0.90)$ and Satisfaction $(r=0.83)$ scales. Support for validity was found as both measures of the SSQ (number of supports, and satisfaction with support) were found to have significant negative correlations with emotional discomfort (e.g. anxiety, depression) and higher social support was correlated positively with positive expectations for the future.

The next scale the Interpersonal Support Evaluation List (ISEL, Cohen \& Hoberman, 1983) has been used in medical and health related areas. This scale was created based on the 
buffering hypothesis, which states that responses to stress are moderated, or buffered by the support we receive. From this hypothesis four subscales of support were created in the original ISEL: tangible, appraisal, self-esteem, and belonging. Tangible support assesses the perceived availability of material aid. Appraisal support assesses the perceived availability of someone to talk to about one's problems. Self-esteem support assesses the perceived availability of others who one favorably compares with, and belonging support assesses the perceived availability of others to socially interact with. Each subscale has 12 -items that are rated on a four-point scale from definitely false to definitely true. This scale was found to have an internal reliability of $\alpha=$ 0.77, across 70 college students ( 27 male, 43 female) and was found to be inversely associated with depressive symptoms. Further strong validity has been shown with significant positive correlations with other support and well-being scales, and significant negative correlations with measures of anxiety, depression, and anger (Cohen et al., 1985).

A shortened version of the scale was created by reducing the number of items for each of the four subscales from 12 to four, creating a 16-item scale with the same four subscales of appraisal support, tangible support, belonging support, and self-esteem support (Payne et al., 2012). This shortened scale was psychometrically tested in a probability sample of 14,257 participants ( $M$ age 57 years, 55\% female) drawn from communities in North Carolina, Mississippi, Minnesota, and Maryland. Internal consistency for the total scale was $(\alpha=0.83)$, with moderate consistency for the subscales $(\alpha=0.53-0.67)$. Evidence for validity was found by moderate correlations with a scale that assessed structural social support $(r=.45)$. Women were found to have higher total scores than men, and scored higher on the belonging and appraisal subscales, while men scored higher on the tangible and self-esteem subscales. Suggesting that women prefer support from those they trust and interact with socially, while men preferred 
support from those they perceived as similar or who had resources they needed. Further, married participants had higher appraisal support than divorced or separated participants and tangible support was highest for married participants than all other groups. Thus, the shortened scale presents a valid and reliable measure of social support and provides insight into different types of social support. This ability to identify different types of support can help understand how peers support each other during a peer support intervention.

\section{Peer Support for Health-behavior Change}

Utilizing peers to provide additional and ongoing support is one promising solution to help individuals maintain their healthier lifestyles. Peer support has been defined as having someone with a similar background and goals provide support, accountability, and coaching around behaviors required for successful change (Boothroyd \& Fisher, 2010). Peer support has been used to help with transitional stressors (e.g. life changes), chronic and acute situation stressors (e.g. diabetes), and for health promotion (Dennis, 2003). This support has been delivered across multiple modes (e.g. phone, in-person), and across multiple contexts (e.g. hospital, in-home). Peers have provided support across three areas: emotional, informational, and appraisal. Emotional support is providing someone the space to discuss personal difficulties that are a common part of life, and act as a resource against the threat these difficulties present to one's self-esteem. Such support helps patients feel accepted, cared for, respected, understood, and valued despite any personal difficulties they may be facing. Informational support involves providing someone with knowledge relevant to problem solving and may include connecting someone with relevant resources in the community, independent assessment of cause of problem, and support in creation of alternative plans of action. A review by Dennis (2003) revealed that most peer support interventions did not incorporate informational support. Finally appraisal or 
affirmational support is focused on generating positive expectations for the future through communicating information that develops positive self-evaluation and affirms emotional, cognitive, and behavioral reactions to dealing with life stressors. A more recent review of peer support literature added mutual reciprocity; that is, the ability of peers who have a similar health problem (e.g. diabetes) to problem solve together, resulting in solutions for both of them (Heisler, 2010). So as one person supports the other, they too are receiving support in the management of their disease. An increased focus on incorporating peer support in health settings has led to the creating of Peers for Progress, a global organization devoted to promoting peer support as a key part of improving health and preventing chronic disease around the world (Boothroyd \& Fisher, 2010). While they are primarily focused on advocating for increased use of peer support, they have created a model to help understand the broad range of roles peers can play in healthcare settings. These roles includes providing support and education about the daily management of chronic conditions, connecting each other to resources, providing emotional and social support, and the ability to provide ongoing support. This model highlights the broad range of roles peers can fill, from providing education about managing a chronic condition to the softer skills required for providing emotional support. Programs can use this model to inform how best to incorporate peer support, do they want peers as educators only, or will peers be best served by serving in multiple roles such as education and emotional support? This model can help to understand the different roles peers can play, beyond the provision of social support.

Before summarizing evidence from the literature, several terms that are used to describe peer support will be reviewed. Peer coaches "are individuals currently facing the health problem who coach one another to promote health-behavior change" (Leahey \& Wing, 2013, p. 928), this type of peer relationship has also been referred to as a peer partner relationship. A peer leader or 
peer mentors someone who has " previously faced the health situation and have demonstrated that they are capable of successfully coping with the situation" (p. 929). Finally, community health worker interventions (CHWI) incorporate peers who have a similar background (e.g. language, ethnicity) or local culture, and some relevant professional training, but who may not be dealing with the same medical condition to provide support.

Peer support and general health improvement. Peer support interventions have been implemented in a wide range of health care settings. A peer support program for individuals with traumatic brain injury (TBI) and their families resulted in improvements in coping with TBI, quality of life, but the interventions did not impact perceptions of social support (Hibbard et al., 2002). This result may point to the multiple ways a peer is able to provide support, which was supported by participants reporting the largest change from the program was an increase in knowledge about TBIs. A train the trainer approach implemented with 64 women in a sorority to deliver a body image program resulted in a reduction in thin-ideal idealization, bulimic pathology, and body dissatisfaction that were similar to prior studies completed by a trained researcher (Greif, Becker, \& Hildebrandt, 2015). This study points to the ability of trained peers to deliver group based programs with similar results to trained professional. A second study of participants recovering from heart failure compared outcomes for those receiving nurse managed care to those receiving support from a partner who was also in the six month intervention (Heisler et al., 2013). Peers received a brief training on basic communication skills, ice breaking activities, a DVD demonstrating peer communication skills and a heart failure self-management booklet to help guide phone calls. Peers were encouraged to talk at least once per week, but results showed that participants struggled to meet these goals, as only $60 \%$ of participants had at least one conversation during the six-month intervention and $82 \%$ made fewer than $50 \%$ of the 
recommended calls. It was hypothesized that the low patient health may have contributed to the lack of engagement in the program. However, despite low number of completed calls similar outcomes were found between the two conditions, as there were no significant differences in heart failure quality of life or social support, and percent of participants who were rehospitalized or who died in the following 12-months.

Several studies have examined the effect peer support has on physical activity of participants. The first study found that those who received monthly phone counseling from a peer leader in their community continued to increase physical activity throughout the second six months of the intervention, while the other two groups saw their activity drop during the second half of the program (Keyserling et al., 2002). Two other studies looking at physical activity also compared results of peer support to participants who received professional support. The first study compared physical activity outcomes of participants across four groups: a non-exercising control group, a group trained by kinesiology students, with specialization in personal training, following a exercise program designed by the research team, a group trained by peer mentors following the same exercise program designed by the research team, and a group trained by peer mentors who created their own exercise program (Dorgo, King, Bader, \& Limon, 2013). There were no education, career, or general fitness requirements for the peer mentors, but an active lifestyle was preferred. Peer mentors completed extensive training over 30 weeks. The first 14 weeks involved participating in the fitness program designed for older adults that the intervention would use, the second half incorporated vigorous physical activity supplemented by activities to improve mentoring skills. In addition, all mentors completed a 40-hour seminar series presented over eight weeks that covered the foundation of fitness and program design. All peer mentors completed the training $(n=36)$ and a select group $(n=5)$ who demonstrated 
superior understanding of concepts presented in seminar and who scored well on final quiz were asked to participate as the independent peer mentors who were in charge of designing and implementing the exercise program for the intervention. A total of 88 participants 60 years or older were assigned to one of the four conditions, and those in an intervention condition received a 14-week exercise program. There were no significant differences in attrition or participation across all conditions, and all three-intervention groups showed significant increases in fitness compared to the control group, with non-significant differences between groups in terms of fitness gains. This study provides support for the ability of trained peer mentors to deliver an exercise program designed by researchers and one designed by themselves, and the results of participants trained by either of these peer groups did not differ from a group trained by students with education and training as a fitness professional. It is important to note that the peers received extensive training, which took a great deal of time, thus limiting the generalizability of this intervention to populations who have time restraints (i.e. those still working).

A second study compared changes in participants step count between group based 16week program delivered by diabetes educators or peer leaders (Tudor-Locke et al., 2009). Peer mentors had completed the same 16-week program, successfully increased their steps per day, and were nominated by professional leader to participate in peer leader training, which covered adult learning, group facilitation skills, and learning the content of the program. Results showed that consistent implementation program across both peer and professional interventions. Further, both groups significantly increased average steps per day from baseline, with no significant difference between peer and professional led groups. Taken together these studies point the viability of peers as a resource to deliver programs and individual coaching designed to improve health through lifestyle change. 
Several studies have studied the effect being a peer supporter has on multiple health outcomes. One such study evaluated a peer leader training program for a group of retired or unemployed individuals with arthritis (Hainsworth \& Barlow, 2001). Results from surveys indicated increases in self-efficacy around pain management, increases in self-management techniques, and a decrease in depressed mood. Interviews were conducted prior to training, and after six-months of working as a peer leader. Prior to peer training participants expected to help others and the community, companionship and peer group support, to learn new coping skills, and to gain personal satisfaction. The main benefits reported at six months included satisfaction from helping others, social interaction with similar others, increased confidence and communication skills, and a feeling of purpose. In addition, many reported making changes to their own lifestyle, such as increasing their exercise, as a result of serving as a peer leader.

An interesting study in China looked at the long-term effects serving as a peer coach. Participants between the ages of 18 and 75 with HbA1c under $8 \%$, who had good understanding of living with diabetes, good communication skills, and a desire to serve were invited to participate in a training program to become peer supporters (Yin et al., 2015). They then completed a 32-hour training program, in four monthly workshops of eight hours, designed to teach them how to provide basic diabetes knowledge and emotionally support to their peers. The training was led by health care experts and involved both didactic and interactive learning activities that covered four main areas: effective communication, diabetes diet review, physical activity training, and behavioral psychology. Finally, at the completion of training each participant underwent a formal evaluation prior to becoming a peer supporter. A group of 59 completed the training (out of 79), of these 33 agreed to be peer supporters, 26 refused, and a separate sample of 60 patients served as a control group. For those who refused $50 \%$ cited a lack 
of time, $19 \%$ a lack of interest, and $15 \%$ did not feel prepared. Those who agreed to be peer supporters were assigned ten patients and asked to provide structured support for one year, with a provision for a voluntary three-year extension. Out of the 33 peer supporters, 21 continued on for the additional three years. Outcomes were assessed at six-months and at four-years. At sixmonths both those who agreed and those who refused to be coaches had stable HbAlc levels, while the comparison group had significantly increased. Surveys revealed that those who completed the training reported significantly higher self-care levels, which may explain why their HbA1c levels remained unchanged. When comparing the two groups who completed the training, there were no significant differences in HbA1c but those who agreed to be supporters had significantly lower fasting blood glucose, and health related quality of life decreased in the refused group while not changing in those who provided support at six-months. While at fouryears $\mathrm{HbA1c}$ had significantly increased for those who refused and for those in the control group, while HbA1c for those who agreed to be peer supporters remained unchanged. This study offers strong evidence that while training to be a peer supporter offers short-term benefits, one receives the greatest benefits long-term by actually serving at a peer supporter.

A second RCT assessed the impact of a peer support program for those providing peer support and those receiving peer support among a group with sclerosis (Schwartz \& Sendor, 1999). They found that those who provided support reported improvement on more outcomes and these improvements had a greater effect size than the patients who received support. When comparing the supporters to the patients, supporters reported over seven times the benefit on well-being, with large improvements in self-esteem, self-awareness, global life satisfaction, personal growth, and purpose in life. These findings support the idea that peer support program 
benefit both the participants and the peers who are providing the coaching or support, and that these results maybe more pronounced for the supporters themselves.

A 2016 systematic review of peer support interventions focused on hard to reach populations (e.g. low socioeconomic status, diagnosed mental health condition) identified 47 studies across a range of health topics (Sokol \& Fisher, 2016). The top three health topics were maternal and child health (26\%), diabetes (17\%), and other chronic disease (15\%), however there was a lack of studies focusing on obesity and weight-loss. 38 studies compared outcomes between groups (randomized or quasi-experimental) and $84 \%$ of these reported significant group differences or changes favoring peer support. They also looked at strategies used by these studies to engage participants. The most common strategy was a focus on trust and respect, with almost $90 \%$ of the studies incorporating this strategy. The two main themes of this strategy were having peers with experience with the pertinent health topic and having similarity between peer supports and participants other than the program's primary health focus. The other two common strategies were flexibility (offering services that respond to needs of participants and their life situation) and user involvement and empowerment (including participants in program development and empowering them to make changes in their lifestyle). A t-test comparing the different strategies and participant retention (a major challenge of studies with hard to reach populations) revealed that only the trust and respect strategy significantly increased participant retention. Overall this review points to the power of peer support can have to improve health outcomes, especially within groups that are typically challenging to reach with standard care. However, one area that requires more research attention is studying the impact peer support has for participants working to manage their weight. 
Peer support within diabetes management. The majority of the studies of peer support have assessed the impact of peer support on health outcomes for patients with type-two diabetes (T2DM). Several of these have trained peers to deliver a group based intervention. The Diabetes Self-Management Program (DSMP) is a six-week program covering a broad range of topics over a two and a half hour group meeting held each week. A intervention in the San Francisco Bay Area was conducted to assess the efficacy of a peer led DSMP (Lorig, Ritter, Villa, \& Armas, 2009). Peer leaders came from the same community that the intervention was held and most had T2DM and were not healthcare professionals. The peer leaders received four days of interactive training that covered in detail the DSMP protocol and how to deliver lectures and lead structured group discussions. Peer leaders used the DSMP protocol as a detailed guide while leading the actual program sessions. Participants in the intervention had T2DM and were 18 years or older and were randomized to either the peer led intervention or to a usual care control group. Participant outcomes were compared across groups at six-months, while the DSMP group was followed for another six-months to gauge what improvements from the DSMP would still be present one-year post baseline. Results at six-months revealed that the intervention group significantly improved communication with their physician, healthy eating, increased reading of food labels, increased aerobic exercise and glucose testing, and decreased depression, however no significant improvement was noted in HbA1c compared to the usual care group. At 12months the intervention group continued to show improvements in depression, communication with physician, healthy eating, and had a mean weight reduction of just over one kilogram. While the intervention appears to effectively increase health-behavior it was odd that HbAlc did not improve, however, participants in both groups had low baseline levels of HbA1c of 6.74 for the usual care group and 6.70 for the intervention group, which may have limited the ability of 
the intervention to detect a change in HbAlc. Overall, the study supports the efficacy of a peer led, community based intervention to lead to improvements in health-behaviors, while the low baseline levels of participant $\mathrm{HbA1c}$ may resulted in nonsignificant changes.

A second peer led, group based randomized controlled trial to manage T2DM was conducted over two-years in Austria (Johansson et al., 2015). Peer leaders were nonprofessionals with T2DM who were recommended by their general practitioners. Peer leaders served a larger role than those in the Lorig et al. (2009) study, as their main roles included organizing group meetings, exercise sessions, logging attendance, facilitating group discussions on diabetes related topics, supporting physically weak participants, and motivating unmotivated participants (Johansson et al., 2015). Professionals trained peers over six, four hours sessions on the following topics: concept of peer support, organization of group meetings, physical activity, recommendation for treatment and management of T2DM, and tools to motivate. Exercise meetings were organized by the peer leaders and met once a week for at least one hour of outdoor activity. Group meetings, also organized by the peer leaders, occurred once a month and focused on personal, social, and emotional topics within the context of diabetes management. These meetings were moderated alternately by the peer leaders or a health professional with a specific content area, which they were asked to talk about with the group. For the peer led groups, peer leaders were provided with a curriculum to help guide the participants through the content area of focus for that month. A total of 143 participants were assigned to the intervention and 185 to control group, who received a standard program for T2DM management. Thus the study looked at the effect of adding a peer support component to program designed for diabetes management. Peer leaders were very engaged in the program, however, participants showed low rates of engagement in the exercise and group meetings. Out of a maximum of 104 exercise 
meetings, participants had a median of 23 sessions attended, while participants participated in only $34 \%$ (four of 14) group sessions. Given this low rate of engagement by participants, and again lower baseline levels of HbAlc, it is not surprising that no significant differences between groups were noted in HbA1c or secondary outcomes like quality of life. The low level of participant engagement may be attributed to addition of the peer support program to a diabetes management program that participants were also participating in. This extra support may have been more than participants were willing to take on. However, despite this the study did show that peer leaders were engaged in the program and committed to a large role in the study over the course of two-years.

While these two studies trained peers to deliver a group based intervention, multiple studies have used peers to provided support on a one-on-one basis. These are split with interventions that used a peer leader model (a peer with diabetes controlled) and a peer partner model (peer who is also currently in the intervention and working to manage their diabetes). The first recruited low-income participants with uncontrolled T2DM from six public health clinics in San Francisco, California (Thom et al., 2013). Participants received either usual care (e.g. education and support from a diabetes nurse, nutritionist) or support from a peer leader. Peers, who had their HbA1c under control (under 8\% over past six-months), were recommended based on their ability to coach by their primary care physician. Peer leaders received 36-hours of training over eight-weeks, and had to pass a written and oral examination to be eligible to serve as a health coach. Training took place in small groups and included education, role playing, and observed practice coaching sessions. The topics of training included communication skills, diabetes self-management skills, tips for providing social and emotional support, and tips for assisting with lifestyle change. Of the 37 peers who enrolled in the training, 26 completed and 
passed examinations, and 24 completed the six-month intervention. Peer leaders were paid 150 dollars for completing the training, and received 25 dollars a month for each client they coached. Peer leaders were given the goal of making contact with their patients at least twice per month, during which they helped their patients design action plans to achieve goals set by the patient. Peer leaders and their patients decided how to best to contact and support each other, with some making contact face to face at the clinic and others providing support over the phone. The majority (over $80 \%$ ) of participants selected their coach based off a brief coach profile, the remaining participants were assigned a coach based on availability. A total of 299 participants were randomized, with 151 receiving usual care and 148 being partnered with a peer coach. Participants had to have an HbAlc over 8\% and speak either English or Spanish, in order to facilitate matching with peer leaders. Peer coaching resulted in a significantly greater drop in HbA1c (-1.07\%) than those who received usual care (-.30\%), with no differences noted between groups in participant attrition. Further, $50 \%$ of those in the coaching condition saw their $\mathrm{HbA} 1 \mathrm{c}$ drop by $1 \%$ or more, significantly greater than the usual care group (32\%). No significant differences were noted between groups at six-month change in cholesterol or BMI. These results lend support to the ability of peer leaders to provide support that results in improved health outcomes.

A follow-up paper analyzed the data from the Thom et al. (2013) study to examine moderators of the peer coaching intervention (Moskowitz et al., 2013). They did not find any moderation of the effect of the peer support intervention on the basis of any demographic or psychological factors, which would suggest that peer coaching would benefit patients from a variety of backgrounds. However, the lack of effect for patient race and ethnicity may be explained by patients self-selecting their coach and by the matching of coach and patient on the 
basis of language. Yet, several significant moderators did emerge. Peer support was found to be significantly more effective for participants with lower levels of self-management and medication adherence. Participants with the highest levels of self-management saw the greatest decreases in HbA1c over the six-month intervention, however, there was no difference between usual care and peer coached participants for those with high levels of self-management. This makes sense, as those with higher levels of self-management may have already learned these skills and thus would see less benefit from having a peer coach. So it may be that one process by which peer coaching impacts participants is by helping patients develop self-management skills. A randomized trial of 628 participants with diabetes in China examined the effect of adding one-on-one peer support to an ongoing diabetes management program (Chan et al., 2014). The ongoing 12-month program provided online support, physician and nurse support, and a personalized risk report every three months that was given to participants during a two-hour, nurse led group empowerment class, with a focus on self-care and attainment of treatment goals. The peer support group received this same class, plus telephone support from a peer leader with well-controlled diabetes (HbA1c under 8\%). Peer leaders received 32-hours of training over four eight hour sessions. Trainings included tutorials, role playing, case sharing, and activities which reinforced the principles of communication and empathic listening, peers were also encouraged to share their own positive experiences managing their diabetes. Peer leaders were assigned 10 patients and instructed to call bi-weekly for three months, monthly for three months, and every other month for the final six-months. The majority of peer leaders completed the program (31 of 33 ), with $90 \%$ maintaining contacts with their peers over the 12 -month program. Yet, results at 12-months revealed both groups significantly lowered their HbA1c levels (-.3\%), however the health improvements for those receiving peer support were not significantly better. Given that 
the peer support group received the same intervention as control, plus peer support, these results suggest that adding peer support to this program did not change health outcomes. Despite these findings participants in the peer support group had significantly fewer hospitalizations, which may indicate that peer support may increase benefits for high risk patients (e.g. comorbid, noncompliant, emotionally distressed) who have higher rates of hospital and emergency care use. In support of this hypothesis post-hoc, exploratory analysis revealed that patients with higher levels of negative emotions (e.g. depression, anxiety) appeared to benefit from the additional peer support with better treatment compliance, improved psychological health, and reduced hospitalizations. While a lack of support was found for peer leaders coaching patients, there is some evidence that perhaps peer support is effective only for those patients experience emotional distress or those with medical history that is considered high risk.

A similar RCT in the United Kingdom (UK) compared telephone peer support from peers who had their diabetes controlled, telephone care from a diabetes nurse specialist, and a control group who were instructed to follow their doctors advice (Dale et al., 2009). Peers received a two-day training focused on patient empowerment, motivational interviewing, and active listening skills; in addition they received books on behavior change and diabetes management. The six-month intervention was implemented across 90 participants with peer support, 44 with the diabetes nurse, and 97 in the control group. Again all three groups significantly reduced HbA1c levels with no significant differences across groups. One possible explanation is the UK rolling out a nationwide financial incentive for meeting health targets for patients with diabetes, thus control group likely received more intense care than they would normally. Semi-structured interviews revealed those receiving peer support found the calls to be professional, friendly, nonjudgmental, and patients appreciated being able to share their own experiences with someone 
who had also struggled with diabetes management. Patients valued receiving supportive comments and feedback from their coach. The peer supporters enjoyed helping others and reported receiving personal benefit from participating (improved knowledge, attitude, skills, relationships, and insights). Further, they reported their own confidence in managing their diabetes had increased, and this led them to start new lifestyle changes for themselves (e.g. increasing exercise). Some of the barriers included finding time to make calls, the number of attempts needed to connect, and keeping calls focused on relevant content.

A unique type of peer support was implemented in a community based, RCT in rural Alabama (Safford et al., 2015). Peer coaches either had diabetes themselves or provided care for someone in their family for a period of at least two years. They received 12-hours of training over two days that covered similar topics to the prior studies reviewed. Each peer leader was paired with six to seven participants from the same community. Peer leaders contacted patients weekly for the fist two months, and monthly for the final eight-months of the ten-month intervention. Participants in the study had diabetes and were interested in receiving selfmanagement help. A total of 168 were assigned to the peer coaching condition, with 192 participants receiving brief diabetes education serving as the controls. Peer leaders had an average of 13.3 phone contacts out of 17 specified by protocol, $32 \%$ contacted participants 17 or more times, $69 \%$ had 10 or more contacts, and eight-percent had zero contact. However, many participants already knew their peer leader and thus interacted with them within the community (e.g. at church), thus the number of contacts likely underestimates the actual number of contacts between peer leaders and participants. Participants in the peer intervention were satisfied with the support they received, with $93 \%$ choosing to focus on weight-loss, $19 \%$ on stress reduction, and $9 \%$ on medication adherence. Results indicated no significant changes in $\mathrm{HbAlc}$ between 
groups, but did find a significant effect for peer coaching on participant change in BMI and quality of life, which would make sense given participant goals.

A six-month randomized controlled trial of a sample of 118 African American's with poor diabetes control were assigned to three conditions: usual care, coaching from a peer leader, or a financial incentive (Long et al., 2012). Those in the usual care condition $(n=39)$ were told their HbAlc level and what it should be. Those in the peer leader condition $(n=38)$ were matched with a peer leader who had well-controlled HbA1c, but had at one point struggled with their diabetes control. Participants were matched with peers of the same gender and who were similar age (within ten-years). Peer leaders completed a one-on-one, hour-long training session that was based on motivational interviewing. Mentors were paid 20-dollars a month if their partner confirmed they spoke at least once a week for the month. Finally, those in the financial incentive received their current $\mathrm{HbAlc}$ levels, provided goals, and told they would be receive 100-dollars if their HbA1c dropped one-percent and 200-dollars if it dropped two-percent. Peer leaders averaged four calls in month one, with $38 \%$ reaching the four call minimum to receive payment, this dropped to two average calls at month six, with only $16 \%$ reaching the four call minimum to receive payment. Those in the peer support group saw a significantly greater drop in their A1c levels (-1.08\%), compared with the standard care (-.01\%) and financial incentive ($.49 \%$ ) groups. Those in the peer support group completed an exit interview about their experiences as a mentor. They appreciated being able to talk with someone who understood their life experience and had a common understanding of what they were going through. This is a benefit that only peers would be able to provide, a nurse who is healthy or who is younger or older than a participant will have inherent challenges in connecting with these patients. Peers do not face this challenge due to their life experience and being matched for age and gender. The 
mentee felt that having a peer who also had diabetes was an important aspect of the peer coaching relationship. They reported the support, education, and ability to commiserate with mentors as the factors they liked best about working with a peer mentor. In addition, mentors also completed an exit interview in which they reported enjoying the process of helping others by teaching them about diabetes. Like their mentees, the mentors also felt it was important that they themselves had at one time struggled to control their HbAlc.

These studies have implemented peer support interventions using a peer leader, or peer who has already successfully managed their diabetes. In contrast, the following studies implemented a peer partner intervention, which partners two peers in the same intervention to provide support to each other as they work to management their health. Two such studies in Africa found mixed results. The first provided psychoeducation led by peer leaders who had managed their diabetes and text message support from a paired peer who was also working towards managing their diabetes (Rotheram-Borus et al., 2012). They found that peer partners exchanged 123 text messages per week, on average, but this did not result in significant changes in $\mathrm{HbA1c}$ or BMI over the six-month intervention. Further, no significant differences were seen in BMI, blood glucose, daily step counts, and sleep quantity between those who exchanged more versus less text messages each week. So while the text messaging support was well received by participants, it did not result in significant improvements in health outcomes. A second study in Uganda paired peer champions with partners in the community (Baumann et al., 2015). Unlike peer leader studies, champions did not have their diabetes managed, and were working to manage their diabetes. Champions were selected because they spoke English and thus were able to receive communication training to provide emotional support and assist with daily management, but there were no language requirements for partners. Champions were asked to 
contact partners each week over a four-month intervention to check-in and set goals, but both champions and partners gave support and answered questions. A high number of peers reported making contact at least weekly (68\%), and no peer had fewer than six contacts over the course of four-months. No differences were found between champions and partners in any health outcomes. Significant changes were found in participant's healthy eating behaviors, while no changes were found in perceived social support, emotional well being, or confidence in selfmanagement. On average $\mathrm{HbA} 1 \mathrm{c}$ across the 41 participants dropped from $11 \%$ to $8 \%$, while there was no change seen in participant BMI. Participants reported receiving helpful advice, encouragement to visit clinic, and having someone to talk to about diabetes care as the largest benefits of having a peer partner (in order of frequency). While they reported not talking enough, being unable to contact peer, being told what to do, and having a peer unmotivated to change as the biggest barriers (in order of frequency). As promising as these results are, this study lacked a control group thus is it hard to determine what effect peer support had on participant health improvements.

A six-week pilot study with 38 male participants whose HbA1c was over eight percent found support for partnering peers in a program to provide support as $80 \%$ of partners spoke at least once a week for two of the six weeks and 56\% spoke four or more times (Heisler \& Piette, 2005). Further, this contact resulted in significant increases in participant self-efficacy to manage their diabetes and self-management behaviors approached significance $(p=.057)$. Finally, follow up surveys with the participants revealed that $90 \%$ of participants would be more satisfied with medical care if peer support was made available, participants found the calls helpful in staying healthy, learning new strategies for managing diabetes, and only seven percent reported that the calls were a challenge. These results suggest that a peer partner intervention is feasible and may 
result in behavior changes that improve health, however, no health outcomes were measured. Building off this pilot a six month randomized controlled trial compared health outcomes of 244 males with A1c over $7.5 \%$ across a group receiving peer support from a partner and those receiving nurse managed care (Heisler et al., 2010). The nurse managed care group attended a one and a half hour session, led by a nurse care manager, in which they reviewed their lab and blood results, gave contact information of their assigned care manager, and provided diabetes self-care materials. The care received in the nurse managed care group represented enhanced standard care. Those in the peer support group attended a three-hour initial session, which mirrored the managed care group for the first half. During the second half participants were paired with another age-matched participant in the program, were provided training on peer communication skills, and given a DVD and a diabetes self-management workbook to help guide peer phone calls. Participants were encouraged to call their partners once per week through a phone system that was used to track calls and also prompt participants to call each other if no contacts had been made over a seven-day period. The phone system revealed that participants called on average 2.4 times in the first month, but this declined to an average of one call for the sixth month, time spent on each call also decreased over the six-months. While it is not clear why the number and duration of decreased over the course of the program, it is possible that some did not need additional support, while others wanted more but were left without it. In addition, 21 participants maintained contact outside of the phone monitoring system, thus their contacts were not tracked. Despite the decreasing phone contacts over the program participants who received support from a peer saw significantly greater drops in HbAlc when compared to those receiving nurse managed care (-.29\% versus $+.29 \%)$. These results suggest that support 
from a peer partner significantly improve participant health and may be more effective than standard care.

Several systematic reviews and meta-analyses have been completed looking at the effect of peer support within diabetes management. The first looked at randomized controlled trials with adults with T2DM which reported $\mathrm{HbA} 1 \mathrm{c}$, and a peer support program was the primary intervention (Qi et al., 2015). Included within peer support intervention were programs with community health workers ( $\mathrm{CHW})$, who may or may not have had T2DM but were similar to participants in other ways. 13 studies met inclusion criteria, with a total of 2,352 participants, $63 \%$ female, and an average age of 57.5 years. The studies ranged from three to 24 months in duration. There was a significant overall effect in favor of peer support versus control group ($.57 \%, \mathrm{CI}-.78,-.36)$. Several interesting findings came out when comparing outcomes between study types. First, a stronger effect for peer support was found for participants whose baseline HbA1c was $8.5 \%$ or higher $(-.78 \%$, CI $-1.06,-.51)$, or between $7.5 \%$ and $8.5 \%(-.76 \%$, CI -1.05 , -.47) as compared to participants with a baseline under $7.5 \%$ who did not significantly improve $(-.08 \%$, CI $-.32, .16)$. Second, participants who were provided an individual peer intervention responded with greater reduction in $\mathrm{HbA} 1 \mathrm{c}(-.91 \%, \mathrm{CI}-1.10,-.71)$ than patients who received a group session $(-.42 \%, \mathrm{CI}-.72,-.11)$ or a combination of group and individual $(-.52 \%, \mathrm{CI}-.66$, .38). Third, a comparison of frequency of contact from high (two or more contacts per month), moderate (one to two contacts per month) and low (less than one contact per month) revealed a significant effect for peer support only for the high $(-.75 \%,-1.29,-.29)$ and moderate $(-.52 \%$, CI $-.60,-.44)$ contact frequency, as interventions with low frequency of contact did not significantly differ from usual care $(-.32 \%$, CI -.74, .09). Finally, a comparison of different modes of peer support (e.g. peer partner versus peer leader versus CHW) did not result in significant differences 
in HbAlc. The authors also highlighted the great variability that exists across studies in terms of quantity, intensity, and focus of peer training.

A second meta-analysis of 20 RCTs with 4,494 participants with T2DM with a peer support intervention revealed a significant decrease in $\mathrm{HbAlc}(-.16 \%, \mathrm{CI}-.25,-.07)$ for participants who received peer support (Zhang et al., 2016). Again subgroup analysis revealed several interesting findings. First, they found that interventions lasting three to six months resulted in largest drop in HbAlc when compared to interventions lasting less than three months, or interventions over 12 months. Peer partner interventions resulted in the largest decrease in HbA1c (-.49\%, CI -.86, -.12), followed by community health worker interventions (-.35\%, CI, $.54,-.16)$, while HbA1c for peer leader interventions did not significantly differ from controls ($.08 \%, \mathrm{CI}-.18, .03)$. Finally they also analyzed the studies across five categories of peer intervention approach. 1) telephone dominant,2) support group, 3) home visit, 4) curriculum only (e.g. peers were trained to deliver a group classes) and 5) curriculum reinforced (e.g. participants received education plus additional support, such as phone calls, from peers). Most studies fell within this final category and resulted in a significant reduction in $\mathrm{HbAlc}(-.24 \%, \mathrm{CI}-.43,-.06)$. The only other category to show a significant effect HbAlc was those involving home visits ($.30 \%, \mathrm{CI}-.53,-.07)$ as interventions using primarily telephone $(-.12 \%, \mathrm{CI}-.31, .07)$, support groups $(-.13 \%$, CI $-.29, .04)$, or only classes $(.01, \mathrm{CI}-.23, .25)$ failed to show a significant effect in favor of peer support. These results suggest that peer partner support programs that are coupled with curriculum-based intervention would appear to be the most effective use of peer support for diabetes management.

Taken together these findings provide strong evidence for the benefit peer support provides to individuals working to manage their T2DM. The research suggests that individual 
support coming from a peer who is also working to manage their diabetes provides a moderate effect size of 0.35 to 0.45 (Qi et al., 2015; Zhang et al., 2016). Further, frequency of contact is an important factor to a peer support intervention being successful, as effective programs provided at least one to two contacts per month, and programs achieving over two contacts per month providing the strongest effect. Several issues emerge from these studies. First, is that the training varied greatly across studies, with some studies providing levels of training that would be unsustainable at a community level due to the amount of time and resources needed. Further, there is no evidence that increased training leads to improved outcomes. In addition, research is needed to better understand how effective matches are made. Finally while there is some overlap between management of diabetes and weight, for example making changes to diet and physical activity, diabetes is a unique medical condition whose outcomes different from weight-loss. For example, those managing diabetes may face additional burdens in managing their diabetes (e.g. managing medication and insulin) that are not part of weigh loss. These challenges could change how peer support is incorporated within weight-loss. Thom et al. (2013) have highlighted the lack of understanding of how peer support can apply to other chronic health conditions, such as weight-loss and obesity management, and outside of controlled trials, as two current issues to move peer support research forward.

Peer support within weight management. There has been a substantial number of studies looking at peer support within diabetes management, however, the same attention has not be given to studying the role peer support can play within weight management, as to date there are only five studies that examined a peer support program looking at weight-loss as the primary outcome. The first study was actually an 16-week intervention for individuals with T2DM who had been recommended to lose weight by their doctor (Wilson $\&$ Pratt, 1987). Participants $(n=$ 
79 , age $M=68.2,80 \%$ female) were recruited from senior centers in a rural area of the United States and randomized by site into one of three conditions. The education only group $(n=19)$ received ten sixty-minute classes led by a registered dietician and one hour of unstructured class time (e.g. where participants could socialize), these classes were delivered weekly for the first eight weeks, and monthly for next two months. The education plus peer support group $(n=32)$ received the same education program, but for the second hour a peer support facilitator led a peer support group. The peer support facilitator received training on group dynamics and was focused on encouraging group interaction. The final group served as a control, receiving no education or support. At eight weeks those in the peer support group lost a significant amount of weight $(-5.5$ pounds, CI $-6.8,-4.2)$, as the education only ( -1.2 pounds) and control ( +0.6 pounds) failed to show significant change in weight. Further a measure of social support showed that the peer support group significantly increased perceived support compared to the education only group, lending support to the role peers can play towards increasing support levels. However, when assessing weight-loss at 16-weeks, after the meeting frequency decreased, none of the groups showed significant weight-loss, as the peer support group had regained the weight they initially lost. These results point the potential of peer support to increase perceived support and to lead to significant weight-losses at eight weeks, however the support is needed longer term to maintain these changes.

A pilot study with obese, predominantly black patients at risk for diabetes, evaluated the feasibility and preliminary outcomes of a six-month weight-loss intervention which included group based education and telephone support from a trained peer coach (Dutton et al., 2015). The six-month intervention was based on the Diabetes Prevention Program (DPP) and the look AHEAD weight-loss trials. Participants were encouraged to work toward a five or ten-percent 
reduction in body weight. Each 60-minute group session included weighing participants and delivering program content by a clinical psychologist, registered dietician, or exercise physiologist with experience delivering behavior weight-loss interventions. Topics covered a range of psychoeducation, nutrition, and physical activity topics geared towards helping participants make lifestyle change. Peer health coaches had successfully lost weight (five percent or more) in a previous program were assigned six to seven patients to support on 10 to 15 minute phone calls following a semi-structured format to enhance their patient's motivation and commitment to weight-related behavior change. Coaches were instructed to contact patients a total of 12 times over the course of the six-month intervention. Peer health coaches received six hours of training covering patient centered communication, goal setting, and motivational interviewing. Training included a combination of didactic presentation and interactive learning activities, such as role-plays. Peer health coaches were compensated 100-dollars for the initial training and for each participant they coached. A total of 32 participants started the intervention, with 28 completing the intervention and achieving a significant weight-loss of five kilograms, or four-percent of their baseline weight. Participants attended, on average, six of the 12 group sessions and completed approximately $40 \%$ of the telephone calls (5 out of 12). Overall, participants were satisfied with the program and the individual coaching they received from their peer coaches, which they felt helped them achieve better health.

A 16-week RCT compared weight-loss across a group of female adolescents who either received a standard cognitive weight-loss intervention $(n=17)$ and a group receiving the same intervention plus enhanced support ( $n=19$, Kulik et al., 2015). The standard weight-loss intervention was the same for both groups, and involved eight face-to-face meetings delivered weekly for first month, biweekly for the second month, and monthly for the final two months. 
After each session the standard group participated in a group activity that reinforced the content of the session, while the enhance group participated in smaller group activities to learn and practice core peer support skills. In addition, the enhanced support group used Facebook to provide support between sessions. Participants in this group were told to chat, on Facebook, for 10 to 15 minutes with either the group leader or a peer in the program, and to check-in with three peers in the program using a structured contact card to guide online discussions. Weight-loss did not significantly differ between groups, with both groups losing over six pounds. The enhanced group showed friend support for healthy eating and exercise significantly increased versus the standard group, but neither of these increases were significantly associated with weight-loss, possibly due to the small sample size. Further, the enhanced group completed an average of six and a half out of 15 contacts with the group leader, which was not associated with weight-loss, however the total number of peer chats completed showed a significant, moderately strong correlation with total weight-loss $(r=-.53, p=.02)$. This result was examined more closely and revealed a significant difference in weight-loss between week four and 16 among participants in the highest tertile for completed check-ins, versus those in the bottom two tertiles. So it appears that peer support is most influential after the first month and requires participants to actively engage with their peers in order to see benefits in weight-loss.

A second RCT randomized 44 obese participants, aged 40 to 60 years, to three weightloss conditions based on coaching from: a peer, a mentor, or a professional (Leahey \& Wing, 2013). Those in the professional coach condition were coached by one of the weight-loss interventionists. All three groups received 12 one-hour meetings as part of a reduced intensity, six-month, weight-loss program. Standard behavioral weight-loss strategies were taught to promote modification of diet and exercise habits. Weight-loss goal of one to two pounds per 
week for each participant. Those in the peer condition were randomly paired to coach and be coached by another member of their weight-loss group. During the first six sessions peers participated in brief relationship-building activities to build cohesive relationships. In addition, during the first two sessions they received peer coach manuals and training in how to be an effective coach, such as learning how to provide support and encouragement, and strategies for promoting behavioral weight-loss. Mentors were recruited from a recent weight-loss program on the basis of successful weight-loss (lost $10 \%$ or more) and maintenance of this loss for 12months. Mentors with similar age and gender characteristics to the participants were also recruited. Prior to first session mentors were given coaching manuals and completed a one-hour training. They were told to be a positive and inspirational role model by drawing on their own experience when providing advice and encouragement and to identify things they had in common with their mentees when discussing how they managed challenges to weight-loss. Each mentor was assigned two participants, and similar to peer the condition they engaged in relationship building activities at the first two meetings. In addition to weight outcomes, they measured autonomous support, which measures the degree to which relationships allow choice. Significant differences in weight outcomes were found, as the participants with professional and peer coaches both lost over nine-percent versus less than six-percent in the mentor-coached group. These differences translate to a moderate effect size for receiving support from a peer versus a mentor $(d=.67)$. In addition, significant differences were found in the percent of participants who achieve weight-loss of ten-percent or higher, again both the peer $(50 \%)$ and professional (56\%) had significantly more participants reaching this benchmark than the mentor group (17\%). When looking at autonomy support, the professional condition showed significantly higher scores than the mentor condition, while the peer group did not differ from 
either group. There was also a significant correlation between autonomy support and weight-loss, with higher autonomy support being associated with greater weight-loss $(r=.38, p=.02)$. It is possible that the lower weight-loss with the mentor group was a result of mentors lower levels of autonomy support. The lower level of autonomy support may have been due to a lack of training and the instructions to share their own positive experience losing weight, which may have limited their ability to connect with their mentees. These results are inline with results from the peer support literature which found that peer leaders were less effective in lowering HbAlc as compared to peer partners (Zhang et al., 2016). In sum, the results "suggest that social influence occurs among peer coaches and among mentors and mentees. Understanding exactly how peer coaches influence on another and how mentors influence their mentees, whether through social support or some other social influence factor, may be important to maximize the effects of lay health coaches for obesity treatment" (Leahey \& Wing, 2013, p. 933).

Another RCT with participants who had achieved a weight-loss of five percent or greater during a eight-week weight-loss trial were randomized into three maintenance conditions: 1) cost-benefit approach with a professional coach, 2) cost benefit approach with a peer coach, and 3) a standard approach (Leahey et al., 2016). The standard approach involved monthly email contact a single hour-long group session that taught traditional evidence based weight maintenance strategies (e.g. self-regulation, self-monitoring), while monthly emails gave tips, recipes, and other relevant information. The cost benefit approach received this same standard approach plus social and financial reinforcement to increase the benefits or rewards of weight maintenance. If participants self-monitored weight, diet, and physical activity for five days and emailed this to their coach they received social reinforcement (e.g. encouragement, support) and received from one to ten-dollars for each week they completed five or more days. The rewards 
were scheduled to be larger $(\$ 10, \$ 8, \$ 5)$ at the beginning of the maintenance program to encourage behavior development, however participants were not told the schedule of payment, only that they could earn up to ten-dollars. Participants could earn a max of 160-dollars if they tracked every week. Each week after submitting their log coaches would inform participants of the amount of money they earned that week and how much they had earned to date in the program. To limit boredom by increasing variety participants were told to track a new behavior every two weeks, (e.g. steps, food). Finally, participants were incentivized to maintain weightloss, with the chance to earn 25-dollars for maintaining their weight-loss in full. For the professional condition a registered dietician with training in behavioral weight control served as the coach. In the peer coach condition participants were paired with a same gender participant in their maintenance arm to provide reciprocal peer support via email. They received one and a half hours of training on how to be a reinforcing and positive coach. Like the professional condition peers provided social reinforcement for completing self-monitoring, self-monitoring goals changed every two weeks to limit boredom, and financial incentives followed the same structure. However, in order to earn financial incentives both peers had to achieve self-monitoring goals for the week and both had to achieve weight maintenance goals in order to receive financial reward for weight maintenance.

Participants ( $n=75$, age $M=48.5$ years, $88 \%$ white) who averaged eight-percent weightloss were randomized into the three conditions (Leahey et al., 2016). No difference in adherence to coaching was seen between groups, and peers and professionals did not differ in percentage of weeks of meeting self-monitoring goals. Both the peer and professional coaching conditions regained significantly less weight than the standard approach, with just under 75-percent of each maintaining their weight at ten-months (within 2.3 kilograms), but they did not significantly 
differ from each other. Further, those in the professional condition were over five times as likely to maintain five-percent loss, while peers were over three times as likely as compared to the standard approach, but these did not significantly differ from each other. Calculated effect size indicates that the peer support resulted in a moderate effect compared to the standard group $(d=$ .67). These results add further support to the ability of peers to provide effective support, along with financial incentives for tracking, that could be equivalent to support provided from a healthcare professional.

Overall, these results show peer support to be a viable alternative to providing professional support and promising solution to increasing the support individuals receive on their journey to better health. However, several areas are in need of further study. First, there is a surprising lack of research examining peer support with weight-loss settings, with the majority of research focusing on diabetes management. We need more research examining the impact peer support can have on those focused on managing their weight. Second, the training peers have received across these studies has varied greatly, thus it remains unknown what training is necessary, or if it is necessary for peer support to be successful. This will be briefly summarized in the next section. Finally, few studies have examined what characteristics or factors lead to more or less successful peer partnerships.

Peer support training. The training provide to peer supporters has varied greatly across studies with training as short as one hour (e.g. Baumann et al., 2015; Leahey \& Wing, 2013) to spanning several days (e.g. Goldman et al., 2015; Thom et al., 2013; Yin et al., 2015), and anywhere in-between. A review of 12 RCTs using volunteer peer coaches with participants with diabetes found that training varied greatly by intensity, duration, and topics covered (Tang et al., 2011). Three levels of training intensity were defined: low (brief three-hour to two and half day 
session with), moderate (four day workshop that covered topics and techniques in greater depth), and high (basic training followed by more specialized training, with much greater depth, e.g. 27 hours covering 18-sessions). They found six studies with low intensity training, three with moderate, and three with high. Of these, four studies included an evaluation of the peer's skills prior to starting to work with participants. They also found a large range in topics taught which ranged from diabetes education, stages of change, patient empowerment, facilitation skills, group dynamics, motivational interviewing, and communication skills.

Peer support matching. Participant matching has varied from using age and gender (e.g. Heisler et al., 2010) to letting participants select their own coach (Moskowitz et al., 2013), or matching based on participant goals (Heisler \& Piette, 2005), but few studies have actually examined how coach and participant characteristics interact to effect the coaching relationship and participant outcomes. The pilot study by Heisler and Piette (2005) matched participants based on whether participants were on insulin therapy, and when possible, on participant's diabetes self-care goals or challenges that they identified on a baseline survey. In this small pilot two of the pairs (out of 20) were unsatisfied with their match because one of the participants was struggling to manage their diabetes while their partner had already mastered it.

A secondary analysis of the peer support program in San Francisco, California by Thom et al. (2013) is the only analysis to explore how different characteristics of peers effect patient outcomes. This study involved peer leaders who had already managed their diabetes providing coaching for participants with the majority of participants selecting their coach from a brief coach profile. The secondary analysis revealed that matching peer coaches by age, gender, ethnicity, or education level did not result in significantly better results in HbAlc over the sixmonth study (Rogers et al., 2014). However, coach BMI, HbA1c, depressive scores, diabetes 
distress, and diabetes self-efficacy for self-management were significantly associated with participant change in $\mathrm{HbA1c}$. First, for coaches with a BMI under 35, lower BMI led to greater improvements in $\mathrm{HbA1c}$, while for coaches with a BMI over 35 a higher BMI was associated with greater improvement in participant $\mathrm{HbAlc}$ levels. So interestingly the further the coaches BMI was from 35, the better the outcomes for the participants they coached. A similar result was found for coach HbA1c levels. For coaches with HbA1c under seven-percent lower levels were associated with improved outcomes, and this trend was reversed for coaches with levels above seven-percent, but the effect was not as strong in this direction. Second, coaches with lower depressive scores had, on average, patients with greater improvements in HbA1c. This finding intuitively makes sense as a depressed coach could easily struggle to motivate their participants, and could get dragged down when their clients share their own struggles. And finally, and perhaps most interestingly, coaches with higher distress and lower sense of self-efficacy around their own diabetes self-management were the most successful. The authors hypothesized that this result may be due to coaches with higher distress being less confident about their selfmanagement, resulting in a collaborative, reciprocal support relationship. It may be that higher distress and lower self-efficacy lead to coaches who work with their participants in a collaborative manner versus taking on a leadership or expert role. This result fits with prior results suggesting that coaches who have already mastered their condition are not perceived as helpful in providing support (Heisler \& Piette, 2005) and those peers who acted as experts were not perceived to be effective in providing support (Hibbard et al., 2002).

\section{Summary and Future Directions}

Weight-loss interventions have been a focus of research for many years and the research has shown that these programs can have a positive influence on an individual's weight-loss. 
However, sustaining weight-loss over the long-term after a program ends remains a challenge. The support individuals receive appears to be an important component of their success, both in the short-term and the long-term, but healthcare professionals simply do not have the time to work one-on-one long-term with all the people who could improve their health through weightloss. Healthcare providers are in need of alternative methods for providing support on an ongoing basis to help individuals sustain change. Incorporating peers into the support process provides an effective and efficient solution to this challenge.

The benefits of peer support fit into theories connecting social support with improved health. Peers can provide direct health effects by sharing information that has gained on their own journey, such as strategies for eating healthier or increasing activity levels while at work. Further, the shared experience of weight-loss helps peers empathize with each other about the challenges associated with weight-loss. This ability to empathize allows peers to serve as buffers to stress and other negative emotions that present challenges to a healthier lifestyle.

The research on peer support has shown that support from a partner, or someone who is currently working to manage their health, is more effective than support from a peer mentor, or someone who has already successfully managed their health. Further these interventions are most effective when they last at least three months and when peers contact each other a minimum of once per month, with best results seen with two or more contacts a month. However, these results are drawn primarily from diabetes research, which presents unique challenges from weight-loss.

Expanding peer support research into weight-loss is needed. With increasing levels of obesity, the link between increased support and improved weight-loss, and the positive results from peer support interventions in diabetes management there is no reason to not expand this 
area of research into weight management. The few studies that have studied peer support within weight-loss have shown favorable results, but more studies are needed to confirm these results. In addition, much of the diabetes research has taken place within a controlled trial, which does not always translate into results in the real world. Thus research focused on evaluating peer support within the community is another area of needed research.

While the literature has shown positive effects on health outcomes for individuals participating in a peer support program, the training given to peers has varied greatly across these studies. Some have included training that spans several days while others are completed in a few hours. Furthermore, the topics of training have varied greatly across studies. It is unclear if training is necessary for peers to provide effective support in improving health outcomes, or if peers on their own, by the nature of their own personal characteristics and backgrounds are able to offer effective support. There has also been a lack of research looking at using online modules to deliver training to peers. This approach would increase the accessibility of training to fit a variety of schedules and reduce costs. A future study could address this by comparing a group who are peer partnered but receive no training, with a group who is not partnered, with a group who receive a short structured online training. This approach would help address what training is needed by first addressing what effect being matched with a peer has on the health outcomes compared with a group who did not receive peer support, and then whether the short training improved the effect of peer support.

In addition, research is needed to better understand how to best pair participants into their peer support relationships. This matching would appear to be a vital component of the peer relationship, yet we know very little about what influences factors such as gender, BMI, ethnicity, depression, anxiety, or social support play on the effectiveness of peer partners. This 
information is especially lacking within weight-loss, as to date no research has assessed what makes peers more or less effective. A study focusing measuring participant and dyadic characteristics of peers and then tracking their satisfaction with the peer relationship and their health outcomes would help identify factors that improve satisfaction and outcomes. Qualitative research can also be used to better understand how peers support each other, and what training may best improve this relationship. Further, exploring what challenges face peers could inform possible training and even identify possible characteristics that can be used in matching peers. 


\section{References}

Abildso, C. G., Schmid, O., Byrd, M., Zizzi, S., Quartiroli, A., \& Fitzpatrick, S. J. (2014). Predictors of weight loss maintenance following an insurance-sponsored weight management program. Journal Of Obesity, 2014(736080), 1-12. https://doi.org/10.1155/2014/736080

Abildso, C. G., Zizzi, S. J., \& Reger-Nash, B. (2010). Evaluating an insurance-sponsored weight management program with the RE-AIM Model, West Virginia, 2004-2008. Preventing Chronic Disease, 7(3), 1-12.

Albarran, C. R., Heilemann, M. V., \& Koniak - Griffin, D. (2014). Promotoras as facilitators of change: Latinas' perspectives after participating in a lifestyle behaviour intervention program. Journal of Advanced Nursing, 70(10), 2303-2313. https://doi.org/10.1111/jan.12383

Baumann, L., Frederick, N., Betty, N., Jospehine, E., \& Agatha, N. (2015). A demonstration of peer support for Ugandan adults with type 2 diabetes. International Journal of Behavioral Medicine, 22(3), 374-383. https://doi.org/10.1007/s12529-014-9412-8

Boothroyd, R. I., \& Fisher, E. B. (2010). Peers for Progress: Promoting peer support for health around the world. Family Practice, 27, 62-68. https://doi.org/10.1093/fampra/cmq017

Brown, S. L., Nesse, R. M., Vinokur, A. D., \& Smith, D. M. (2003). Providing social support may be more beneficial than receiving it: Results from a prospective study of mortality. Psychological Science, 14(4), 320-327. https://doi.org/10.1111/1467-9280.14461

Chan, J. C. N., Sui, Y., Oldenburg, B., Zhang, Y., Chung, H., Goggins, W., ... Fisher, E. (2014). Effects of telephone-based peer support in patients with type 2 diabetes mellitus receiving 
integrated care: A randomized clinical trial. JAMA Internal Medicine, 174(6), 972-981. https://doi.org/10.1001/jamainternmed.2014.655

Christakis, N. A., \& Fowler, J. H. (2007). The spread of obesity in a large social network over 32 years. New England Journal of Medicine, 357(4), 370-379. https://doi.org/10.1056/NEJMsa066082

Cohen, S., \& Hoberman, H. M. (1983). Positive events and social supports as buffers of life change stress. Journal of Applied Social Psychology, 13(2), 99-125.

Cohen, S., Mermelstein, R., Kamarck, T., \& Hoberman, H. M. (1985). Measuring the functional components of social support. In I. G. Sarason \& B. R. Sarason (Eds.), Social Support: Theory, Research and Applications (pp. 73-94). The Hague, The Netherlands: Martinus Nijhoff. https://doi.org/10.1007/978-94-009-5115-0_5

Dale, J., Caramlau, I., Sturt, J., Friede, T., \& Walker, R. (2009). Telephone peer-delivered intervention for diabetes motivation and support: The telecare exploratory RCT. Patient Education and Counseling, 75(1), 91-98. https://doi.org/10.1016/j.pec.2008.09.014

Dansinger, M. L., Tatsioni, A., Wong, J. B., Chung, M., \& Balk, E. M. (2007). Meta-analysis: The effect of dietary counseling for weight loss. Annals of Internal Medicine, 147(1), 4150.

Dennis, C.-L. (2003). Peer support within a health care context: A concept analysis. International Journal of Nursing Studies, 40(3), 321-332. https://doi.org/10.1016/S00207489(02)00092-5

DiMatteo, M. R. (2004). Social support and patient adherence to medical treatment: A metaanalysis. Health Psychology, 23(2), 207-218. 
Dombrowski, S. U., Knittle, K., Avenell, A., Araújo-Soares, V., \& Sniehotta, F. F. (2014). Long term maintenance of weight loss with non-surgical interventions in obese adults: Systematic review and meta-analyses of randomised controlled trials. BMJ, 348(g2646), 1-12. https://doi.org/10.1136/bmj.g2646

Dorgo, S., King, G. A., Bader, J. O., \& Limon, J. S. (2013). Outcomes of a peer mentor implemented fitness program in older adults: A quasi-randomized controlled trial. International Journal of Nursing Studies, 50(9), 1156-1165. https://doi.org/10.1016/j.ijnurstu.2012.12.006

Dutton, G. R., Phillips, J. M., Kukkamalla, M., Cherrington, A. L., \& Safford, M. M. (2015). Pilot study evaluating the feasibility and initial outcomes of a primary care weight loss intervention with peer coaches. The Diabetes Educator, 41(3), 361-368. https://doi.org/10.1177/0145721715575356

Feeney, B. C., \& Collins, N. L. (2015). A new look at social support: A theoretical perspective on thriving through relationships. Personality and Social Psychology Review, 19(2), 113147. https://doi.org/10.1177/1088868314544222

Franz, M. J., VanWormer, J. J., Crain, A. L., Boucher, J. L., Histon, T., Caplan, W., ... Pronk, N. P. (2007). Weight-loss outcomes: a systematic review and meta-analysis of weight-loss clinical trials with a minimum 1-year follow-up. Journal of the American Dietetic Association, 107(10), 1755-1767.

Fryar, C. D., Carroll, M. D., \& Ogden, C. L. (2016). Prevalence of overweight, obesity, and extreme obesity among adults aged 20 and over: United States, 1960-1962 through 2013-2014 (Health E-Stats) (p. 6). National Center for Health Statistics. Retrieved from https://www.cdc.gov/nchs/data/hestat/obesity_adult_13_14/obesity_adult_13_14.pdf 
Goldman, M. L., Ghorob, A., Hessler, D., Yamamoto, R., Thom, D. H., \& Bodenheimer, T. (2015). Are low-income peer health coaches able to master and utilize evidence-based health coaching? Annals of Family Medicine, 13, 36-41. https://doi.org/10.1370/afm.1756

Greaves, C. J., Sheppard, K. E., Abraham, C., Hardeman, W., Roden, M., Evans, P. H., \& Schwarz, P. (2011). Systematic review of reviews of intervention components associated with increased effectiveness in dietary and physical activity interventions. BMC Public Health, 11(1), 119-130. https://doi.org/10.1186/1471-2458-11-119

Greif, R., Becker, C. B., \& Hildebrandt, T. (2015). Reducing eating disorder risk factors: A pilot effectiveness trial of a train-the-trainer approach to dissemination and implementation. International Journal of Eating Disorders, 48(8), 1122-1131. https://doi.org/10.1002/eat.22442

Hainsworth, J., \& Barlow, J. (2001). Volunteers' experiences of becoming arthritis selfmanagement lay leaders: "It's almost as if I've stopped aging and started to get younger!" Arthritis Care \& Research, 45(4), 378-383. https://doi.org/10.1002/15290131(200108)45:4<378::AID-ART351>3.0.CO;2-T

Heaney, C. A., \& Israel, B. A. (2008). Social networks and social support. In K. Glanz, B. K. Rimer, \& K. Viswanath (Eds.), Health behavior and health education: theory, research, and practice (4th ed., pp. 189-210). San Francisco, CA: Jossey-Bass.

Heisler, M. (2010). Different models to mobilize peer support to improve diabetes selfmanagement and clinical outcomes: Evidence, logistics, evaluation considerations and needs for future research. Family Practice, 27, 23-32. https://doi.org/10.1093/fampra/cmp003 
Heisler, M., Halasyamani, L., Cowen, M. E., Davis, M. D., Resnicow, K., Strawderman, R. L., ... Piette, J. D. (2013). Randomized controlled effectiveness trial of reciprocal peer support in Heart FailureClinical perspective. Circulation: Heart Failure, 6(2), 246-253. https://doi.org/10.1161/CIRCHEARTFAILURE.112.000147

Heisler, M., \& Piette, J. D. (2005). "I help you, and you help me”: Facilitated telephone peer support among patients with diabetes. Diabetes Educator, 31(6), 869-879.

Heisler, M., Vijan, S., Makki, F., \& Piette, J. D. (2010). Diabetes control with reciprocal peer support versus nurse care management: A randomized trial. Annals of Internal Medicine, 153(8), 507-515. https://doi.org/10.7326/0003-4819-153-8-201010190-00007

Hibbard, M. R., Cantor, J., Charatz, H., Rosenthal, R., Ashman, T., Gundersen, N., ... Gartner, A. (2002). Peer support in the community: Initial findings of a mentoring program for individuals with traumatic brain injury and their families. Journal of Head Trauma Rehabilitation, 17(2), 112-131.

Huisman, S. D., Gucht, V. D., Dusseldorp, E., \& Maes, S. (2009). The effect of weight reduction interventions for persons with Type 2 Diabetes: A meta-analysis from a self-regulation perspective. The Diabetes Educator, 35(5), 818-835.

https://doi.org/10.1177/0145721709340929

Hwang, K. O., Ottenbacher, A. J., Green, A. P., Cannon-Diehl, M. R., Richardson, O., Bernstam, E. V., \& Thomas, E. J. (2010). Social support in an Internet weight loss community. International Journal of Medical Informatics, 79(1), 5-13. https://doi.org/10.1016/j.ijmedinf.2009.10.003

Johansson, T., Keller, S., Winkler, H., Ostermann, T., Weitgasser, R., \& Sönnichsen, A. C. (2015). Effectiveness of a peer support programme versus usual care in disease 
management of diabetes mellitus type 2 regarding improvement of metabolic control: A cluster-randomised controlled trial. Disease Markers, 1-10.

https://doi.org/10.1155/2016/3248547

Keyserling, T. C., Samuel-Hodge, C. D., Ammerman, A. S., Ainsworth, B. E., HenríquezRoldán, C. F., Elasy, T. A., ... Bangdiwala, S. I. (2002). A randomized trial of an intervention to improve self-care behaviors of African-American women with type 2 diabetes: Impact of physical activity. Diabetes Care, 25(9), 1576-1583.

Knowler, W. C., Barrett-Connor, E., Fowler, S. E., Hamman, R. E., Lachin, J. M., Walker, E. A., \& Nathan, D. M. (2002). Reduction in the incidence of type 2 diabetes with lifestyle intervention or Metformin. New England Journal of Medicine, 346(6), 393-403. https://doi.org/10.1056/NEJMoa012512

Kulik, N., Ennett, S. T., Ward, D. S., Bowling, J. M., Fisher, E. B., \& Tate, D. F. (2015). Brief report: A randomized controlled trial examining peer support and behavioral weight loss treatment. Journal of Adolescence, 44, 117-123. https://doi.org/10.1016/j.adolescence.2015.07.010

Leahey, T. M., Fava, J. L., Seiden, A., Fernandes, D., Doyle, C., Kent, K., ... Wing, R. R. (2016). A randomized controlled trial testing an internet delivered cost-benefit approach to weight loss maintenance. Preventive Medicine, 92, 51-57. https://doi.org/10.1016/j.ypmed.2016.04.013

Leahey, T. M., \& Wing, R. R. (2013). A randomized controlled pilot study testing three types of health coaches for obesity treatment: Professional, peer, and mentor. Obesity, 21(5), 928934. https://doi.org/10.1002/oby.20271 
Long, J. A., Jahnle, E. C., Richardson, D. M., Loewenstein, G., \& Volpp, K. G. (2012). Peer mentoring and financial incentives to improve glucose control in African American veterans: A randomized trial. Annals of Internal Medicine, 156(6), 416-424. https://doi.org/10.7326/0003-4819-156-6-201203200-00004

Lorig, K., Ritter, P. L., Villa, F. J., \& Armas, J. (2009). Community-based peer-led diabetes selfmanagement: A randomized trial. Diabetes Educator, 35(4), 641-651. https://doi.org/10.1177/0145721709335006

Moskowitz, D., Thom, D. H., Hessler, D., Ghorob, A., \& Bodenheimer, T. (2013). Peer coaching to improve diabetes self-management: Which patients benefit most? Journal of General Internal Medicine, 28(7), 938-942. https://doi.org/10.1007/s11606-013-2367-7

Payne, T. J., Andrew, M., Butler, K. R., Wyatt, S. B., Dubbert, P. M., \& Mosley, T. H. (2012). Psychometric evaluation of the Interpersonal Support Evaluation List-Short Form in the ARIC study cohort. Sage Open, 2(3), 2158244012461923.

Peirson, L., Fitzpatrick-Lewis, D., Ciliska, D., Usman Ali, M., Raina, P., \& Sherifali, D. (2015). Strategies for weight maintenance in adult populations treated for overweight and obesity: A systematic review and meta-analysis. CMAJ Open, 3(1), 47-54. https://doi.org/10.9778/cmajo.20140050

Qi, L., Liu, Q., Qi, X., Wu, N., Tang, W., \& Xiong, H. (2015). Effectiveness of peer support for improving glycaemic control in patients with type 2 diabetes: A meta-analysis of randomized controlled trials. BMC Public Health, 15(1), 1-11. https://doi.org/10.1186/s12889-015-1798-y

Radloff, L. S. (1977). The CES-D scale: A self-report depression scale for research in the general population. Applied Psychological Measurement, 1(3), 385-401. 
Ramage, S., Farmer, A., Apps Eccles, K., \& McCargar, L. (2013). Healthy strategies for successful weight loss and weight maintenance: A systematic review. Applied Physiology, Nutrition, and Metabolism, 39(1), 1-20.

Rieger, E., Treasure, J., Murray, K., \& Caterson, I. (2017). The use of support people to improve the weight-related and psychological outcomes of adults with obesity: A randomised controlled trial. Behaviour Research and Therapy, 94, 48-59.

Rogers, E. A., Hessler, D. M., Bodenheimer, T. S., Ghorob, A., Vittinghoff, E., \& Thom, D. H. (2014). Diabetes peer coaching: Do "better patients" make better coaches? The Diabetes Educator, 40(1), 107-115. https://doi.org/10.1177/0145721713513178

Rotheram-Borus, M. J., Tomlinson, M., Gwegwe, M., Comulada, W. S., Kaufman, N., \& Keim, M. (2012). Diabetes buddies peer support through a mobile phone buddy system. The Diabetes Educator, 38(3), 357-365. https://doi.org/10.1177/0145721712444617

Safford, M. M., Andreae, S., Cherrington, A. L., Martin, M. Y., Halanych, J., Lewis, M., ... Richman, J. S. (2015). Peer coaches to improve diabetes outcomes in rural Alabama: A cluster randomized trial. Annals of Family Medicine, 13, S18-S26. https://doi.org/10.1370/afm.1798

Sarason, I. G., Levine, H. M., Basham, R. B., \& Sarason, B. R. (1983). Assessing social support: The Social Support Questionnaire. Journal of Personality and Social Psychology, 44(1), 127-139. https://doi.org/10.1037/0022-3514.44.1.127

Schwartz, C. E., \& Sendor, R. M. (1999). Helping others helps oneself: Response shift effects in peer support. Social Science \& Medicine, 48(11), 1563-1575.

https://doi.org/10.1016/S0277-9536(99)00049-0 
Sokol, R., \& Fisher, E. (2016). Peer support for the hardly reached: A systematic review. American Journal of Public Health, 106(7), e1-e8. https://doi.org/10.2105/AJPH.2016.303180

Tang, T. S., Ayala, G. X., Cherrington, A., \& Rana, G. (2011). A review of volunteer-based peer support interventions in diabetes. Diabetes Spectrum, 24(2), 85-98.

Thom, D. H., Ghorob, A., Hessler, D., De Vore, D., Chen, E., \& Bodenheimer, T. A. (2013). Impact of peer health coaching on glycemic control in low-income patients with diabetes: A randomized controlled trial. Annals of Family Medicine, 11(2), 137-144. https://doi.org/10.1370/afm.1443

Tsai, A. G. (2005). Systematic review: An evaluation of major commercial weight loss programs in the United States. Annals of Internal Medicine, 142(1), 56. https://doi.org/10.7326/0003-4819-142-1-200501040-00012

Tudor-Locke, C., Lauzon, N., Myers, A. M., Bell, R. C., Chan, C. B., McCargar, L., ... Rodger, N. W. (2009). Effectiveness of the First step Program delivered by professionals versus peers. Journal Of Physical Activity \& Health, 6(4), 456-462.

Wang, J., Shih, P. C., \& Carroll, J. M. (2015). Life after weight loss: Design implications for community-based long-term weight management. Computer Supported Cooperative Work (CSCW), 24(4), 353-384. https://doi.org/10.1007/s10606-015-9226-5

Wilson, W., \& Pratt, C. (1987). The impact of diabetes education and peer support upon weight and glycemic control of elderly persons with Noninsulin Dependent Diabetes Mellitus (NIDDM). American Journal of Public Health, 77(5), 634-635. 
Wing, R. R., \& Jeffery, R. W. (1999). Benefits of recruiting participants with friends and increasing social support for weight loss and maintenance. Journal of Consulting and Clinical Psychology, 67(1), 132.

Yin, J., Wong, R., Au, S., Chung, H., Lau, M., Lin, L., ... Chan, J. C. N. (2015). Effects of providing peer support on diabetes management in people with type 2 diabetes. The Annals of Family Medicine, 13(Suppl 1), S42-S49. https://doi.org/10.1370/afm.1853

Zhang, X., Yang, S., Sun, K., Fisher, E. B., \& Sun, X. (2016). How to achieve better effect of peer support among adults with type 2 diabetes: A meta-analysis of randomized clinical trials. Patient Education and Counseling, 99(2), 186-197. https://doi.org/10.1016/j.pec.2015.09.006

Zimet, G. D., Dahlem, N. W., Zimet, S. G., \& Farley, G. K. (1988). The Multidimensional Scale of Perceived Social Support. Journal of Personality Assessment, 52(1), 30-41.

Zizzi, S., Abildso, C., Henderson, N., \& Cobb, K. (2014). The West Virginia PEIA Weight Management Program: An Innovative approach to obesity prevention and treatment in Appalachian communities. In V. M. Brennan, S. K. Kumanyika, R. E. Zambrana, V. M. Brennan, S. K. Kumanyika, \& R. E. Zambrana (Eds.), Obesity interventions in underserved communities: Evidence and directions. (pp. 282-289). Baltimore, MD, US: Johns Hopkins University Press. 


\section{Appendix B - Peer support enrollment script}

\section{Email for enrollment:}

The PEIA weight management program is piloting a new optional service in which two members are partnered with each other to provide support in reaching their goals. Are you interested in learning more about this optional service?

\section{Script for phone call:}

In this role you will be asked to support each other by helping motivate, supporting each others goals and helping create plans and strategies for achieving them, and finally, to challenge each other to extend beyond your comfort zone, We ask that you contact your partner once per week as this has been shown to result in the largest health improvements. You can contact each other through whatever means is easiest for the two of you, such as over the phone, via text, email, or in-person. You are not expected to provide counseling, nutrition, or exercise advice. If challenges come up in these areas please contact myself, or an administrator at your site who can help connect you with the appropriate person to help.

Any questions?

\section{Email for matching of peers:}

$\mathrm{Hi}$ $\&$

Happy e-troduction. You are being partnered up to provide support to each other by helping motivate, set goals, develop plans to achieve them, and to support each other to try new things. The goal is to try and contact each other at least once per week as this has been shown to lead to the best results. Below is contact information for each of you: 
Participant 1 contact info

Participant 2 contact info

Please reach out with any questions or concerns.

Best, 


\section{Appendix C - Data collection timeline}

\begin{tabular}{l|cccc} 
Time (month) & $\mathbf{0}$ & $\mathbf{1}$ & $\mathbf{2}$ & $\mathbf{3}$ \\
\hline Intervention & $\mathrm{X}$ & $\mathrm{X}$ & $\mathrm{X}$ \\
Baseline psychosocial data & $\mathrm{X}$ & $\mathrm{X}$ & $\mathrm{X}$ & $\mathrm{X}$ \\
Weight & $\mathrm{X}$ & $\mathrm{X}$ & $\mathrm{X}$ & $\mathrm{X}$ \\
Contact frequency & $\mathrm{X}$ & & $\mathrm{X}$ \\
Contact type & $\mathrm{X}$ & & & \\
Peer satisfaction & $\mathrm{X}$ & & & \\
ISEL & & & $\mathrm{X}$ & \\
\hline Comparison group & $\mathrm{X}$ & $\mathrm{X}$ & $\mathrm{X}$ \\
\hline Baseline psychosocial data & & & \\
\hline Weight & & & \\
\hline
\end{tabular}

Table 1. Proposed schedule for data collection across intervention and comparison group. 


\section{Appendix D - CES-Depression Scale}

Below is a list of the ways you might have felt or behaved over the past week. Please enter the number that best represents how often you felt this way during the past week.

$0=$ Rarely or non of the time (less than 1 day in the last week) $1=$ Some of the time (1-2 days in the last week)

$2=$ Much of the time (3-4 days in the last week)

$3=$ Most or all of the time (5-7 days in the last week)

1. I was bothered by things that usually don't bother me.

2. I did not feel like eating; my appetite was poor.

3. I felt that I could not shake off the sad feelings even with help from family and friends.

4. I felt that I was just as good as other people.

5. I had trouble keeping my mind on what I was doing.

6. I felt depressed.

7. I felt that everything I did was an effort.

8. I felt hopeful about the future.

9. I thought my life had been a failure.

10. I felt fearful.

11. My sleep was restless.

12. I was happy.

13. I talked less than usual.

14. I felt lonely. 
15. People were unfriendly.

16. I enjoyed life.

17. I had crying spells.

18. I felt sad.

19. I felt that people dislike me.

20. I could not get going.

Items 4, 8, 12, and 16 are reversed scored (Radloff, 1977). 


\section{Appendix E - Interpersonal Support Evaluation Scale}

Interpersonal Support Evaluation Scale-Short Form (Payne, et al., 2012).

Four-point scale for each item: definitely true, probably true, probably false, definitely false.

Scored 0-3.

1. Most of my friends are more interesting than I am.

2. When I feel lonely, there are several people I can talk to.

3. I often meet or talk with family or friends.

4. I feel like I am not always included by my circle of friends.

5. There really is no one who can give me an objective view of how I'm handling my problems.

6. If I were sick and needed someone (friend, family member, or acquaintance) to take me to the doctor, I would have trouble finding someone.

7. If I were sick, I could easily I find someone to help me with my daily chores.

8. When I need suggestions on how to deal with a personal problem, I know someone I can turn to.

9. I don't often get invited to do things with others.

10. Most of my friends are more successful at making changes in their lives than I am.

11. If I had to go out of town for a few weeks, it would be difficult to find someone who would look after my house or apartment (the plants, pets, garden, etc.).

12. There is really no one I can trust to give me good financial advice.

13. I am more satisfied with my life than most people are with theirs.

14. It would be difficult to find someone who would lend me their car for a few hours.

15. There is at least one person I know whose advice I really trust. 
16. I have a hard time keeping pace with my friends.

Reverse scored: 1, 4, 5, 6, 9, 10, 11, 12 14, 16

Appraisal support subscale (AP): 5, 8, 12, 15

Tangible asset support subscale (TA): 6, 7, 11, 14

Belonging support subscale (BE): 2, 3, 4, 9

Self-esteem support subscale (SE): 1, 10, 13, 16 


\section{Appendix F - Final HLM Model Structure and Interpretation}

\section{Level 1: time variables (negative time variable, centered at month 3)}

Weight_change $_{t i j}=\pi_{0 i j}+\pi_{1 i j}$ NegTime $_{t i j}+e_{t i j}$

\section{Level 2: individual level variables}

$$
\begin{aligned}
\pi_{0 i j}=\beta_{00 j}+ & \beta_{01 j} \text { Age } *_{i j}+\beta_{02 j} \text { BaselineBMI } *_{i j}+\beta_{03 j} \text { Gender }_{i j}+\beta_{04 j} \text { PeerSpt }_{i j} \\
& +\beta_{05 j} \text { PS } *_{i j}+r_{0 i j} \\
\pi_{1 i j}=\beta_{10 j}+ & \beta_{11 j} \text { Age } *_{i j}+\beta_{12 j} \text { BaselineBMI } *_{i j}+\beta_{13 j} \text { Gender }_{i j}+\beta_{14 j} \text { PeerSpt }_{i j} \\
& +\beta_{15 j} \text { PS } *_{i j}+r_{1 i j}
\end{aligned}
$$

PS: propensity score.

PeerSpt: dummy code for if participant was partnered with a peer.

* indicates variable was centered at the grand mean.

\section{Variable interpretation}

$\pi_{0}=$ average total weight change at month three for a female participant, with age, baseline BMI, and PS at the grand mean, and who was not partnered with a peer.

$\pi_{1}=$ instantaneous rate of weight change.

$\beta_{00}=$ average weight change at 3 months (time 0 ) for one who wasn't partnered with a peer, and whose PS, baseline BMI, age, and PS are at the grand mean. Negative value indicate weight-loss at 3-months.

$\beta_{01}=$ effect on total weight change for each 1 unit change in age from the grand mean.

$\beta_{02}=$ effect on total weight change for each 1 unit change in BMI from the grand mean.

$\beta_{03}=$ effect on total weight change for male participants.

$\beta_{04}=$ effect on total weight change for those partnered with a peer. 
$\beta_{05}=$ effect on total weight change for each 1 unit change in PS from the grand mean.

$\beta_{10}=$ instantaneous/linear rate of weight change at time 0 for a female participant who was not partnered with a peer, holding all other variables at grand mean. Negative value indicates weightloss, positive value indicates weight gain.

$\beta_{11}=$ effect of 1 unit change in age from grand mean on linear rate of weight change.

$\beta_{12}=$ effect of 1 unit change in baseline BMI from grand mean on linear rate of weight change.

$\beta_{13}=$ effect of being male on linear rate of weight change.

$\beta_{14}=$ effect of being partnered with a peer on linear rate of weight change

$\beta_{15}=$ effect of 1 unit change in PS from grand mean on linear rate of weight change. 


\section{Appendix G - Propensity Score Analysis Full Output}

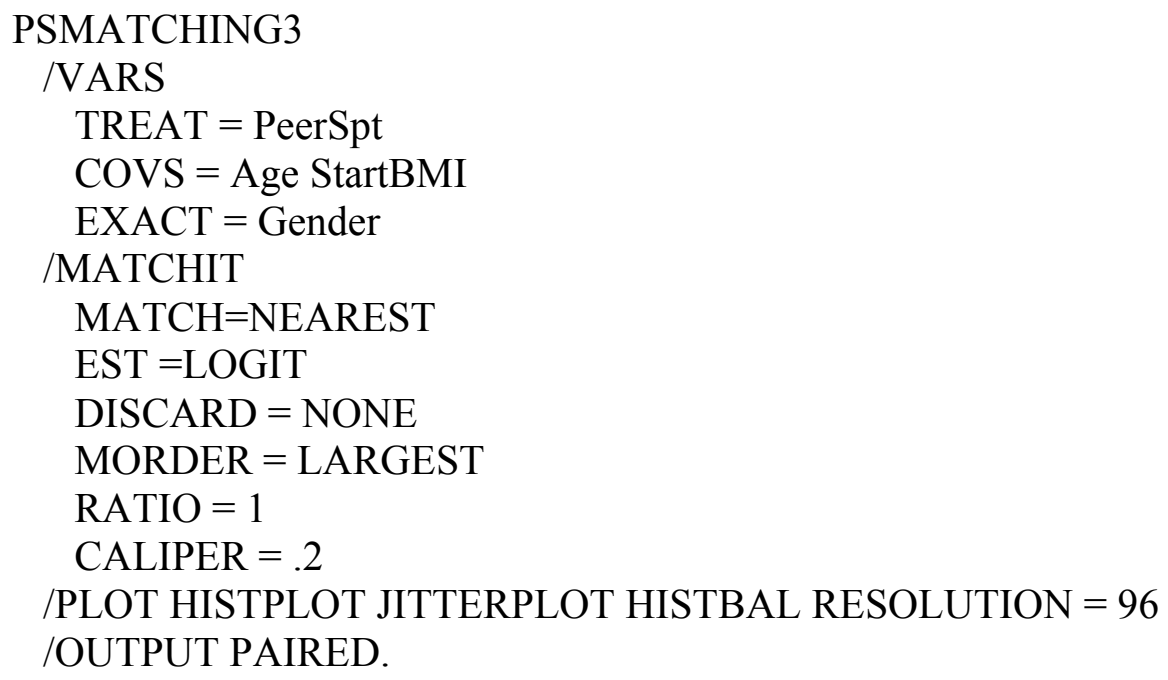

Propensity Score Matching

\begin{tabular}{|c|c|c|c|c|c|c|c|}
\hline \multirow[b]{3}{*}{ Subsamples } & \multicolumn{6}{|c|}{ Sample Sizes } & \multirow{3}{*}{$\begin{array}{c}\text { Discarded } \\
\text { Control }\end{array}$} \\
\hline & \multicolumn{2}{|c|}{ All } & \multicolumn{2}{|c|}{ Matched } & \multicolumn{2}{|c|}{ Unmatched } & \\
\hline & Control & Treated & Control & Treated & Control & Treated & \\
\hline (all cases) & 2032 & 31 & 30 & 30 & 2002 & 1 & 0 \\
\hline
\end{tabular}

\section{Sample Sizes}

Discarded

Subsamples

Treated

\section{Overall balance test (Hansen \&}

Bowers, 2010)

\begin{tabular}{lrll|} 
& chisquare & df & p.value \\
\hline (all cases) & 1.034 & 2.000 & .596 \\
\hline
\end{tabular}

\section{Relative multivariate imbalance L1}

(lacus, King, \& Porro, 2010)

\begin{tabular}{lrrr} 
& Before matching & After matching \\
\hline (all cases) & .740 & .300 \\
\hline
\end{tabular}




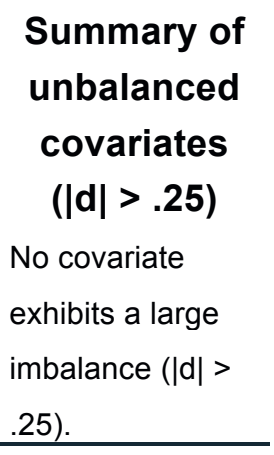

\section{RGraph}

\begin{tabular}{|c|c|c|}
\hline \multicolumn{3}{|c|}{ Notes } \\
\hline \multicolumn{2}{|c|}{ Output Created } & 01-AUG-2018 15:08:17 \\
\hline \multicolumn{3}{|l|}{ Comments } \\
\hline \multirow[t]{5}{*}{ Input } & Data & $\begin{array}{l}\text { E:IPEIAIPeer } \\
\text { supportITrim|BAMF4_PeerMe } \\
\text { rgedPSM2015_Present_trim. } \\
\text { sav }\end{array}$ \\
\hline & Active Dataset & DataSet1 \\
\hline & Filter & $<$ none $>$ \\
\hline & Weight & $<$ none $>$ \\
\hline & Split File & $<$ none $>$ \\
\hline \multirow[t]{2}{*}{ Resources } & Processor Time & 00:00:00.00 \\
\hline & Elapsed Time & 00:00:00.00 \\
\hline
\end{tabular}




\section{Distribution of Propensity Scores}

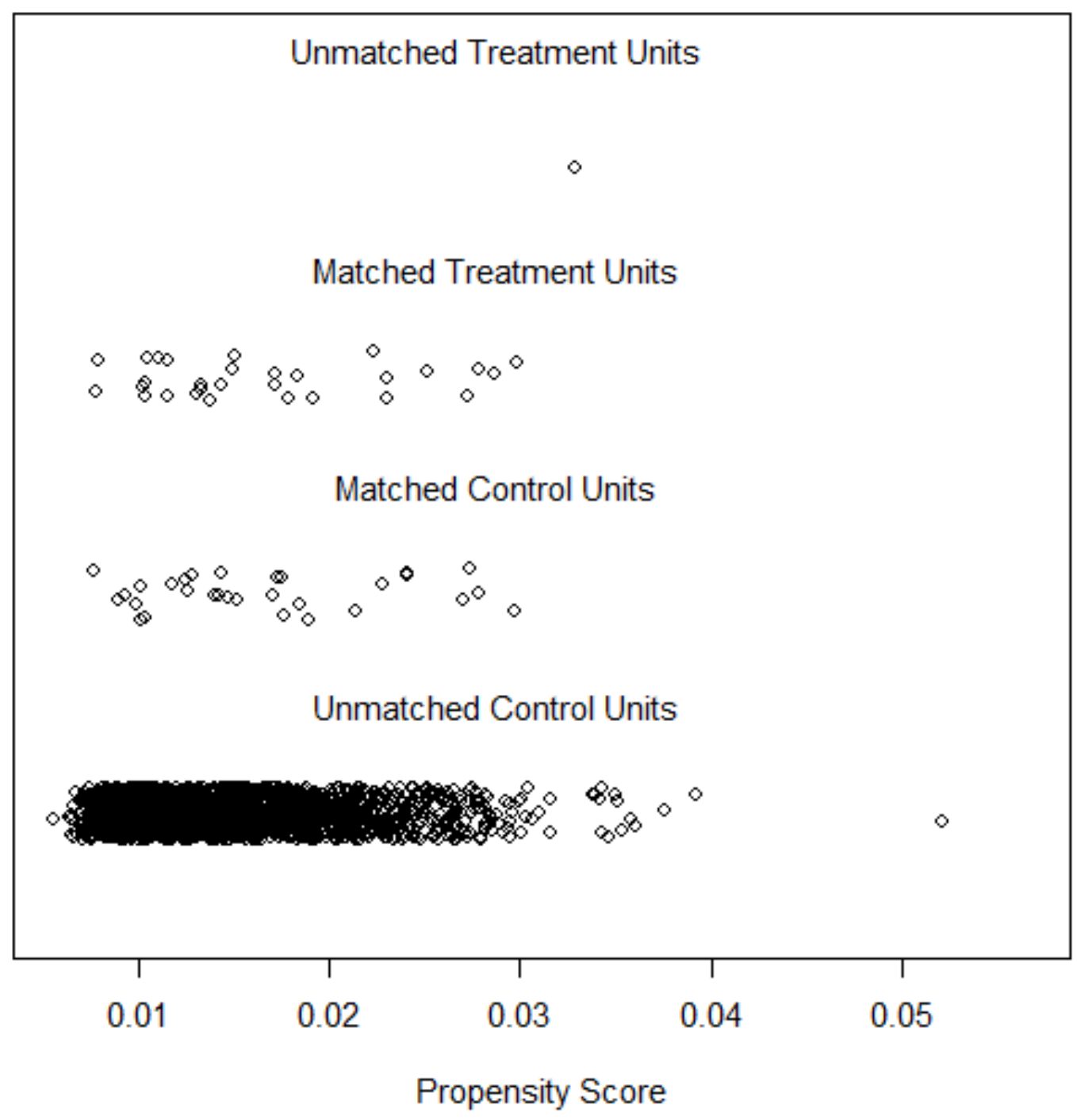

\section{RGraph}

Notes 


\begin{tabular}{llll}
\hline Comments & & \\
Input & Data & $\begin{array}{l}\text { E:IPEIAIPeer } \\
\text { supportITrimIBAMF4_PeerMe } \\
\text { rgedPSM2015_Present_trim. } \\
\text { sav }\end{array}$ \\
\cline { 3 - 4 } & & DataSet1 & \\
\cline { 2 - 3 } & Active Dataset & $<$ none $>$ & \\
\cline { 2 - 3 } & Filter & $<$ none $>$ & $00: 00: 00.02$ \\
\cline { 2 - 3 } & Weight & $<$ none $>$ & $00: 00: 00.02$ \\
\hline Resources & Split File & & \\
\cline { 2 - 3 } & Processor Time & & \\
& Elapsed Time & & \\
\hline
\end{tabular}

Unmatched Treated

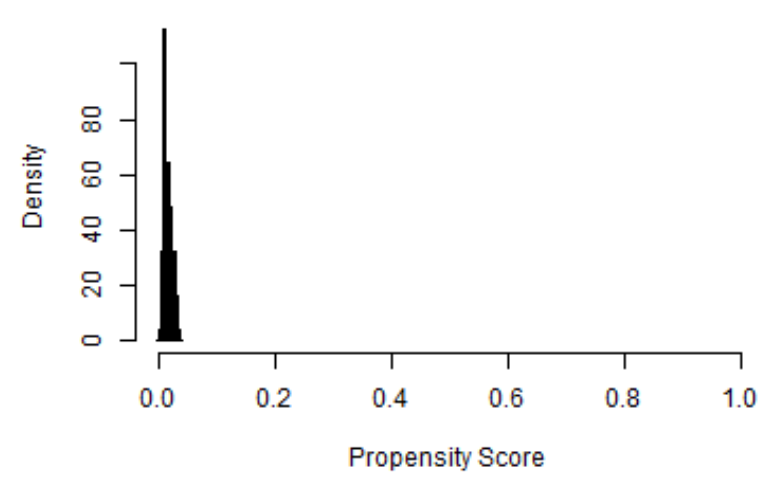

Unmatched Control

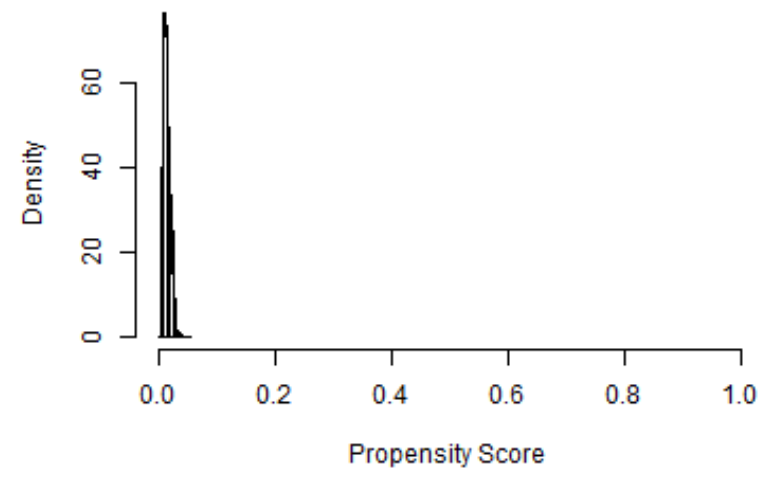

Matched Treated

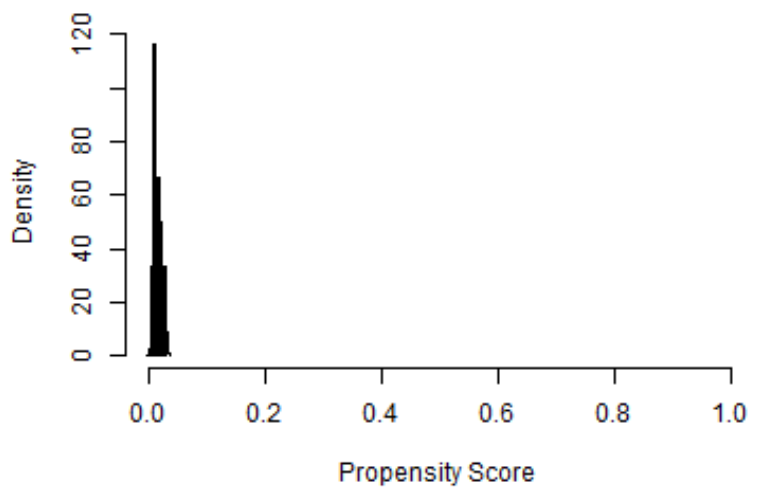

Matched Control

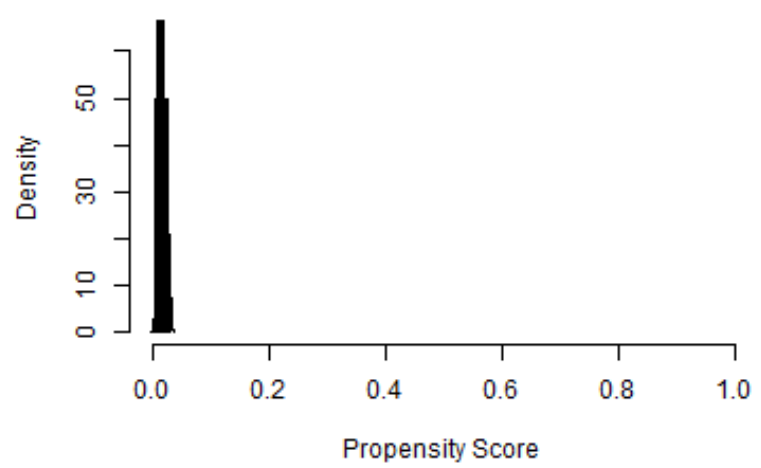

\section{RGraph}




\begin{tabular}{|c|c|c|}
\hline \multicolumn{3}{|c|}{ Notes } \\
\hline \multicolumn{2}{|c|}{ Output Created } & 01-AUG-2018 15:08:17 \\
\hline \multicolumn{3}{|l|}{ Comments } \\
\hline \multirow[t]{5}{*}{ Input } & Data & $\begin{array}{l}\text { E:IPEIAIPeer } \\
\text { supportITrimIBAMF4_PeerMe } \\
\text { rgedPSM2015_Present_trim. } \\
\text { sav }\end{array}$ \\
\hline & Active Dataset & DataSet1 \\
\hline & Filter & $<$ none $>$ \\
\hline & Weight & $<$ none $>$ \\
\hline & Split File & $<$ none $>$ \\
\hline \multirow[t]{2}{*}{ Resources } & Processor Time & 00:00:00.00 \\
\hline & Elapsed Time & 00:00:00.00 \\
\hline
\end{tabular}




\section{Standardized differences before matchin!}

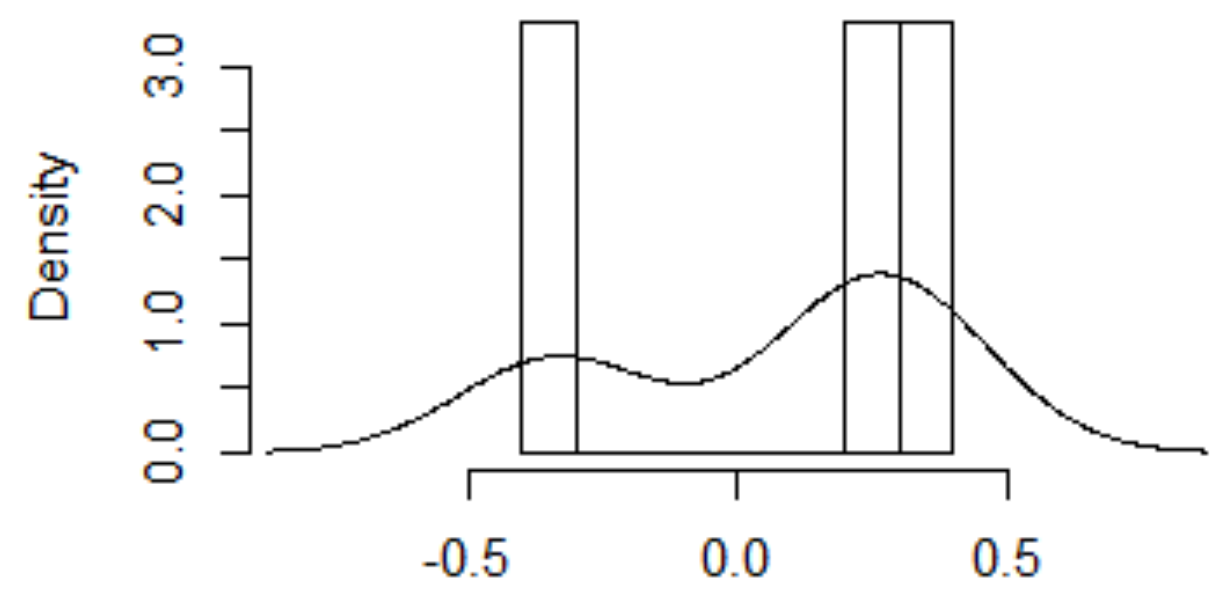

Std. difference

\section{Standardized differences after matching}
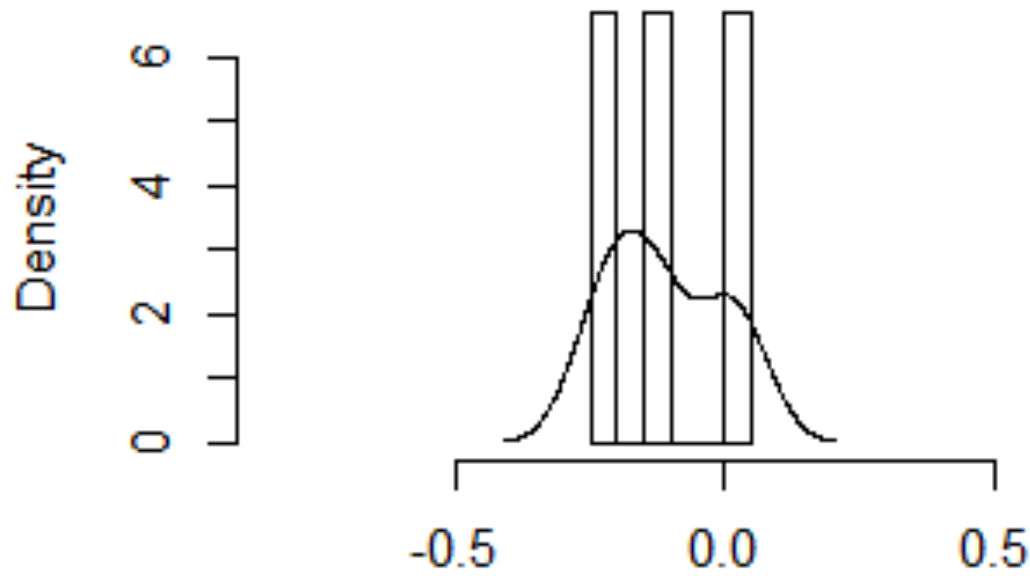

Std. difference 


\section{Appendix H - HLM Full Output}

Program: $\quad$ HLM 7 Hierarchical Linear and Nonlinear Modeling

Authors: $\quad$ Stephen Raudenbush, Tony Bryk, \& Richard Congdon

Publisher: $\quad$ Scientific Software International, Inc. (c) 2013

hlm@ssicentral.com

www.ssicentral.com

Module: HLM2.EXE (7.03.21710.17001)

Date: $\quad 12$ September 2018, Wednesday

Time: $\quad 11: 34: 6$

Specifications for this HLM2 run

Problem Title: no title

The data source for this run = peer2level.mdm

The command file for this run $=\mathrm{C}: \backslash U$ sers $\backslash$ jsingels $\backslash A p p D a t a \backslash L o c a l \backslash T e m p \backslash$ whlmtemp.hlm

Output file name $=\mathrm{V}: \backslash$ Secure $\backslash$ PEIA Grant $\backslash$ Research $\backslash$ Spencer research $\backslash$ Peer

support\Dissertation $\backslash$ Outcomes $\backslash$ Final files $\backslash H L M \backslash 2$ level model $\backslash \mathrm{hlm} 2 . \mathrm{html}$

The maximum number of level- 1 units $=240$

The maximum number of level-2 units $=60$

The maximum number of iterations $=100$

Method of estimation: restricted maximum likelihood

The outcome variable is WTCHG

Summary of the model specified

Level-1 Model

WTCHG $_{t i}=\pi_{0 i}+\pi_{l i}^{*}\left(N E G T I M E_{t i}\right)+e_{t i}$

Level-2 Model

$$
\begin{aligned}
\pi_{0 i} & =\beta_{00}+\beta_{01} *\left(A G E_{i}\right)+\beta_{02} *\left(\operatorname{STARTBMI}_{i}\right)+\beta_{03} *\left(\text { GENDER }_{i}\right)+\beta_{04} *\left(\text { PEERSPT }_{i}\right) \\
& +\beta_{05} *\left(\mathrm{PS}_{i}\right)+r_{0 i} \\
\pi_{1 i} & =\beta_{10}+\beta_{11} *\left(\text { AGE }_{i}\right)+\beta_{12} *\left(\operatorname{STARTBMI}_{i}\right)+\beta_{13} *\left(\text { GENDER }_{i}\right)+\beta_{14} *\left(\text { PEERSPT }_{i}\right) \\
& +\beta_{15} *\left(\mathrm{PS}_{i}\right)+r_{1 i}
\end{aligned}
$$

AGE STARTBMI PS have been centered around the grand mean.

Mixed Model

$W T C H G_{t i}=\beta_{00}+\beta_{01} * A G E_{i}+\beta_{02} * S T A R T B M I_{i}+\beta_{03} * G E N D E R_{i}$

$+\beta_{04} * P E E R S P T_{i}+\beta_{05} * P S_{i}$

$+\beta_{10} * N E G T I M E_{t i}+\beta_{11} * A G E_{i}^{*} N E G T I M E_{t i}+\beta_{12} * S T A R T B M{ }_{i}^{*} N E G T I M E_{t i}+$

$\beta_{13} * G E N D E R_{i}^{*} N E G T I M E_{t i}$

$+\beta_{14} * P E E R S P T_{i}^{*} N E G T I M E_{t i}+\beta_{15} * P S_{i}^{*} N E G T I M E_{t i}$

$+r_{0 i}+r_{l i} * N E G T I M E_{t i}+e_{t i}$

Final Results - Iteration 2044 
Iterations stopped due to small change in likelihood function

$\sigma^{2}=3.65465$

$\tau$

INTRCPT1, $\pi_{0} \quad 43.19071 \quad 13.60885$

NEGTIME, $\pi_{l} \quad 13.60885 \quad 4.28832$

$\tau$ (as correlations)

INTRCPT $1, \pi_{0} \quad 1.000 \quad 1.000$

NEGTIME, $\pi_{l} \quad 1.000 \quad 1.000$

\begin{tabular}{ll}
\hline Random level-1 coefficient & Reliability estimate \\
\hline INTRCPT $1, \pi_{0}$ & 0.944 \\
NEGTIME, $\pi_{1}$ & 0.854 \\
\hline
\end{tabular}

The value of the log-likelihood function at iteration $2044=-5.769778 \mathrm{E}+002$

Final estimation of fixed effects:

\begin{tabular}{llllll}
\hline Fixed Effect & Coefficient & $\begin{array}{l}\text { Standard } \\
\text { error }\end{array}$ & $t$-ratio & $\begin{array}{c}\text { Approx. } \\
d . f .\end{array}$ & $p$-value \\
\hline For INTRCPT1, $\pi_{0}$ & & & & & \\
INTRCPT2, $\beta_{00}$ & -4.778155 & 1.268295 & -3.767 & 54 & $<0.001$ \\
AGE, $\beta_{01}$ & 0.216082 & 0.373681 & 0.578 & 54 & 0.565 \\
STARTBMI, $\beta_{02}$ & -0.114616 & 0.283230 & -0.405 & 54 & 0.687 \\
GENDER, $\beta_{03}$ & -1.088632 & 4.068788 & -0.268 & 54 & 0.790 \\
PEERSPT, $\beta_{04}$ & 1.137795 & 1.762833 & 0.645 & 54 & 0.521 \\
PS, $\beta_{05}$ & 412.624592 & 852.068516 & 0.484 & 54 & 0.630 \\
For NEGTIME slope, $\pi_{1}$ & & & & \\
INTRCPT2, $\beta_{10}$ & -1.621230 & 0.420097 & -3.859 & 54 & $<0.001$ \\
AGE, $\beta_{11}$ & 0.082899 & 0.123774 & 0.670 & 54 & 0.506 \\
STARTBMI, $\beta_{12}$ & -0.050713 & 0.093814 & -0.541 & 54 & 0.591 \\
GENDER, $\beta_{13}$ & -0.748367 & 1.347702 & -0.555 & 54 & 0.581 \\
PEERSPT, $\beta_{14}$ & 0.565908 & 0.583902 & 0.969 & 54 & 0.337 \\
PS, $\beta_{15}$ & 166.990434 & 282.230114 & 0.592 & 54 & 0.557 \\
\hline
\end{tabular}

The robust standard errors cannot be computed for this model.

Final estimation of variance components

\begin{tabular}{llllll}
\hline \multirow{2}{*}{ Random Effect } & $\begin{array}{c}\text { Standard } \\
\text { Deviation }\end{array}$ & $\begin{array}{l}\text { Variance } \\
\text { Component }\end{array}$ & d.f. & $\chi^{2}$ & $p$-value \\
\hline INTRCPT1, $r_{0}$ & 6.57196 & 43.19071 & 54 & 963.16012 & $<0.001$ \\
NEGTIME slope, $r_{l}$ & 2.07083 & 4.28832 & 54 & 355.30234 & $<0.001$ \\
level-1, $e$ & 1.91171 & 3.65465 & & & \\
\hline
\end{tabular}

Statistics for current covariance components model

Deviance $=1153.955576$

Number of estimated parameters $=4$ 